\title{
COMETARY ACTIVITY, DISCRETE OUTGASSING AREAS, AND DUST-JET FORMATION
}

\author{
Z. SEKANINA \\ Jet Propulsion Laboratory \\ California Institute of Technology \\ 4800 Oak Grove Drive \\ Pasadena, California 91109 \\ U.S.A.
}

\begin{abstract}
Major progress has recently been achieved in the understanding of the coma morphology of dust comets. The conceptual models for the various types of feature observed in the coma (jets, spirals, halos, fans, etc.), their computer simulation, and the hydrodynamic models for jet formation are critically reviewed, and evidence for anisotropic, strongly collimated flows of ejecta emanating from discrete active regions (vents) on the rotating cometary nuclei is presented. The techniques employed to generate synthetic comet images that simulate the observed features are described and their relevance to the primary objectives of coma-morphology studies is exemplified. Modelling of temporal variations in the water emission from discrete active regions suggests that production curves asymmetric with respect to perihelion should be commonplace. Critical comparisons are offered with the activity profiles of Encke's comet and with light curves of disappearing comets and comets that undergo outbursts. Discussed next is the potential evolutionary significance of nuclear shape, the fractional area of the surface that is active, the vents' erosion rate, and available information on their activation, aging, dormancy, migration, rejuvenation, and extinction. Recent developments in the understanding of the processes that cause the nongravitational perturbations of cometary motions are reviewed and the observed discontinuities are identified with birth of new sources and/or deactivation of old vents.
\end{abstract}

\section{Introduction}

Information on structural detail in the heads of comets has slowly been accumulating over centuries. For example, the often publicized rendition of Halley's comet by Hevelius (1682) shows a bright jet extending from the nucleus toward the Sun and curving to a side. Numerous drawings of coma features in dozens of comets-some accompanied by micrometric measurements - can be found in the scientific journals and monographs published in the 19th century (for examples and references, cf. Rahe, Donn, and Wurm 1969). As photography was gradually replacing visual telescopic observations of comets in the late 1800 s and the early 1900 s, the amount of information on coma morphology began to decrease appreciably. The observers, fascinated by the faint, rapidly evolving tail structures, shifted their attention to fast, large-field cameras and long exposure times. As a result, the scales of most photographic images were inadequate for coma-morphology studies and the near-nucleus region was almost always hopelessly overexposed. Even on those rare photographs of the early 20th-century comets that do have appropriate exposures and scales, 
coma features do not show well because the low-contrast photographic emulsions could not compete with the human eye in detecting boundaries of changing intensity gradients. Frustrations experienced in coma-morphology studies of that era are amply documented in Bobrovnikoff's (1931) extensive investigation of Halley's comet at its 1910 apparition.

The renewed interest in coma morphology since the 1970 s has been stimulated by several factors: (1) the appearance of prominent spiral structure in the coma of comet Bennett 1970 II; (2) ongoing efforts to refine Whipple's (1950, 1951) comet model, accelerated by growing suspicion that dust-coma morphology and its rapid variations may be associated with - and therefore diagnostic of - such physical properties of the cometary nucleus as rotation and gross surface topography; (3) major advances in detector performance, especially the availability of the charge coupled device (CCD) arrays; (4) the development of sophisticated computer-processing techniques designed to carry out digital image enhancement of structural detail both on photographs (after their appropriate digitization) and on modern CCD images of comets; and (5) the pressing need for a better understanding of the nucleus environment in the era of space exploration of comets, in order to minimize hazards to spacecraft and instrumentation, to optimize the performance of the onboard experiments, and to provide a sound basis for the science definition of cometary missions.

Reviewed below is a broad range of topics, from conceptual models for dust-coma morphology to hydrodynamic models of jet formation; from descriptions of synthetic comet images that simulate the ground-based imaging observations to the production curves for comets with isolated sources (active regions) on their surfaces; and from physical characteristics of cometary outbursts and nongravitational perturbations of the orbital motions of comets to the implications that the existence of active centers has for the evolution of cometary nuclei.

Two related areas of investigation not reviewed here, except for remarks closely connected to discussed topics, are: (1) the closeup observations of the dust coma of Halley's comet from spacecraft, a problem that is covered by $\mathrm{K}$. Szegö in his paper in these proceedings (see also, e.g., Keller et al. 1987); and (2) the image processing techniques, whose excellent summary review was presented by Larson (1986).

\section{Modern Studies of Coma Morphology}

Early investigations of the morphology of cometary heads were limited to descriptions and measurements of the observed features. Although Bessel $(1836 \mathrm{a}, \mathrm{b})$ called attention to what is today perceived as overwhelming evidence for anisotropic outgassing from comets and pioneered the interpretation of nongravitational perturbations of comets' orbital motions as an effect of recoil forces that are exerted on the nucleus by the mass ejected asymmetrically with respect to perihelion, his ideas (now, in modern form, universally accepted) had been abandoned or completely ignored for more than a century, until the dawn of the icy-conglomerate model's era (Dubiago 1948; Whipple 1950). Not until Whipple's finding that anisotropic sublimation of water provides an adequate momentum-transfer mechanism and that rotation plays a role in the momentum's distribution had a major contribution been offered since Bessel's times.

Sharp jets and other distinct features on the sunward side of the coma are-with rare exceptions-composed of dust. For early observations this was inferred: (1) from the color of the structures, which was usually reported as yellow, dark yellow, or reddish; (2) from 
observed changes in their shape and configuration, which implied subkilometer-per-second particle velocities; and (3) especially from their well-defined contours and slow rate of fading, which were indicative of highly organized motions, in contrast to semichaotic molecular motions. More recently, the dust nature of most jet phenomena was confirmed by the direct imaging of jets in the various continuum passbands. Coherent motions of dust particles (except for grains smaller than a few tenths of a micron in diameter; cf. Sec. 2.3) through the coma allow one to argue that their momentum acquired upon ejection from the nucleus is preserved to the extent that quantitative dynamical modelling of each jet feature should result in identification of its source's location on the nucleus by extrapolating particle trajectories back to the surface. Although the gas distribution in the coma was also found to display structural nonuniformities (cf. Sec. 2.2 for references), these data are less suitable for extracting information on discrete active regions.

\subsection{CONCEPTUAL MODELS IN THE PRE-HALLEY ERA}

Quantitative analyses aimed at interpreting discrete dust-coma features in comets began with a paper by Larson and Minton (1972) on comet Bennett 1970 II (see also Larson 1978). They used the lawn-sprinkler analogy to explain the spiral dust jets in the comet's head and to show that the jet curvature readily yields the comet's spin rate when Earth is located in the direction of one of the rotation poles and the particle ejection velocity is known. Several years later, Whipple $(1978,1980)$ employed the separations between expanding dust halos in the coma to derive the rotation parameters of comets Donati 1858 VI and SchwassmannWachmann 1 . He concluded that the activity of the two comets was largely confined to single areas that apparently migrated over the nucleus surface. At about the same time, observations of sunward fan-shaped heads of several comets were studied in an effort to gain information on the nucleus spin vector and thermal lag in sublimation (Sekanina 1979, 1981a). From temporal variations in the configuration of sharp, narrow dust jets in comet Swift-Tuttle, it was possible to determine the comet's complete spin vector, to establish that the jets were products of relatively brief bursts of dust from several discrete regions whose total area was not more than 1 percent of the nucleus surface, and to map the source distribution function (Sekanina 1981b).

\subsection{MODELS FOR COMET HALLEY AND EVIDENCE OF OUTGASSING ANISOTROPY}

In 1983, a new conceptual model of dust emission from comets was developed and subsequently applied to more than 50 coma features detected on Halley's high-resolution photographs that were taken in May-June 1910 and recently computer processed (Sekanina and Larson 1983, 1984, 1986a; Larson and Sekanina 1984a, 1985). Dust features observed for more than one day were found to begin characteristically as spiral jets that "unwind" from the nuclear condensation on the sunward side of the comet's head and to develop rapidly into expanding quasi-parabolic envelopes. It was demonstrated that this evolution is diagnostic of a collimated flow of dust ejecta emanating essentially continuously from discrete regions that get activated on the dayside of the spinning nucleus but become dormant as they rotate out of sunlight. The highly structured computer-simulation code, which generates synthetic images of a dust comet, employs a trial-and-error approach to match the rate of expansion of the feature boundaries observed as they propagate through the coma and to map the parent sources on the nucleus surface, with the comet's spin vector determined in the process (Sec. 3.2). The model was applied to Halley's comet on the assumption of 
an inertially fixed spin axis. In retrospect, with the complex rotation of the comet's nucleus at least conceptually understood or constrained (cf. M.J.S. Belton's paper in these proceedings), the problem of Halley's source function of dust in 1910 should be revisited.

Other models of anisotropic dust emission have been proposed for Halley's comet, most of them primarily aimed at interpreting the results of the various in situ dust-impact experiments on board the VEGA-1, VEGA-2, and Giotto spacecraft (McDonnell et al. 1987; Mazets et al. 1987; Simpson et al. 1987; Vaisberg et al. 1987) and of the Giotto RadioScience Experiment (Edenhofer et al. 1987). Carey et al. (1984) considered, among other scenarios, jetlike streams of dust particles of a certain mass ejected from a discrete region on the rotating nucleus to show the expected intercept patterns for the Giotto spacecraft as a function of the spin rate. Fertig and Schwehm's (1984) model for Halley's dust environment, which had originally been designed to study the spatial-density distribution of dust particles in the coma brought about by smooth emission variations over the nucleus surface (cf. Divine and Newburn 1983), was subsequently upgraded to generate synthetic images of jets (Massonne 1986; Massonne et al. 1986; Massonne and Grün 1986; Grün, Massonne, and Schwehm 1987). A special feature of this model is the linearization of the solution in terms of the ratio between the nucleus-particle distance and heliocentric distance. At the cost of a negligible loss of accuracy in most instances, this model substantially reduces the amount of computer time used compared with that needed for the rigorous solution. Chakaveh et al.'s (1986) model for dust emissions from comet Halley is based on the Finson-Probstein (1968) approach and assumes azimuthal symmetry with respect to the comet-Sun line and a maximum emission rate at the subsolar point. Highly simplified models for interpreting local peaks on the dust-flux curves measured by the in situ dust-impact experiments were proposed by Pankiewicz and McDonnell (1986) and by Vaisberg et al. (1987); these models neglect radiation pressure effects on particles and therefore have only limited validity.

The most sophisticated analysis of the Halley data from the in situ dust experiments was offered by Rabinowitz (1988). Based primarily on information from the DUCMA detectors on board VEGA-1 and VEGA-2 (Simpson et al. 1987), Rabinowitz's major conclusions are as follows: (1) the flux enhancements of $\sim 10^{-13} \mathrm{~g}$ dust particles are explained by the spacecraft's encounters with spiral jets, in which the particles were accelerated to a narrow range of velocities that decrease with increasing mass; (2) some of the spiral jets were crossed by the spacecraft more than once, which allows mapping of the dust sources on the nucleus surface; (3) some of the jets encountered by VEGA-1 and VEGA-2 turn out to have identical source locations on the nucleus (indicating that their activity persisted over at least a few rotations) and they correlate with the active regions observed by the multicolor camera on board Giotto and with the imaging results obtained from the ground; and (4) the source map derived from the 1986 in situ data correlates with the distribution of the active regions that was reported by Sekanina and Larson (1986a) from their analysis of the 1910 images, which suggests that the major sources could persist for a full revolution about the Sun.

Only limited attention has so far been paid to modelling the evolution of jet features on the high-resolution ground-based images of Halley's comet taken during the 1986 apparition. Grün et al. (1986) argued that a persistent southward dust jet, observed repeatedly in November 1985, originated near the sunlit rotation pole of the nucleus. Sekanina and Larson (1986b) modelled three jets observed from the ground on 27-28 February and 1415 March 1986, finding tentative correlations both with the source map from 1910 and with a jet imaged by the Giotto camera at the time of encounter. Independent studies of 
Halley's ground-based imaging observations made in the same period of time were reported by Cosmovici et al. (1986) and by Ip et al. (1987). It should be pointed out that, without exception, all the efforts aimed at mapping dust sources on Halley's nucleus have been frustrated by the incomplete understanding of the comet's rotation and that all the results achieved so far in these efforts should be considered as tentative.

Since cometary rotation is discussed separately by Belton in his paper, this review only comments on direct interaction between rotation and activity. Because of limited space, a large amount of other information that is likewise relevant to the problems of outgassing anisotropy in comets, but is not-within the scope of this review-directly related to the topics of immediate interest, is only mentioned in passing. These issues include, among others: (1) gas jets and shells (e.g., A'Hearn et al. 1986a,b; Schlosser, Schulz, and Koczet 1986; Sekanina 1986a; Combi 1987; Cosmovici et al. 1987, 1988; Larson et al. 1987; Hoban et al. 1988; Schulz and Schlosser 1989); (2) color properties and their variations (e.g., Hartmann, Cruikshank, and Degewij 1982; Hartmann and Cruikshank 1984; Jewitt and Meech 1986, 1988a; Campins, A'Hearn, and McFadden 1987; Hartmann, Tholen, and Cruikshank 1987; Millis, A'Hearn, and Campins 1988; A'Hearn et al. 1989; Hanner and Newburn 1989; Hoban et al. 1989); (3) asymmetries in the $\mathrm{H}_{2} \mathrm{O}$ and $\mathrm{OH}$ coma brightness and velocity distributions (e.g., Snyder et al. 1976; Bockelée-Morvan and Gérard 1984; Cordes et al. 1986; de Pater, Palmer, and Snyder 1986; de Pater et al. 1986; Larson et al. 1986; Larson, Mumma, and Weaver 1987; Schloerb, Claussen, and Tacconi-Garman 1987; Palmer, de Pater, and Snyder 1989); (4) variations in the ultraviolet emissions (e.g., Feldman, A'Hearn, and Millis 1984; Feldman, Weaver, and Festou 1984; Festou et al. 1986; Kaneda et al. 1986; Feldman et al. 1987); (5) infrared-flux variations, especially in the thermal region (e.g., Gehrz and Ney 1986; Lynch et al. 1986; Campins et al. 1987a,b; Hanner et al. 1987b); (6) radar observations of the nuclei and large-size ejecta (e.g., Kamoun et al. 1982; Goldstein, Jurgens, and Sekanina 1984; Campbell, Harmon, and Shapiro 1989; Harmon et al. 1989); (7) various aspects of the nature and thermal history of the nuclei, including heat-transport processes (e.g., Shulman 1972; Smoluchowski 1981b, 1982, 1986, 1989; Klinger 1983, 1986; Gombosi and Houpis 1986; Weissman 1986, 1987); and (8) laboratory simulation experiments (e.g., Grün et al. 1987, 1989; Ibadinov, Aliev, and Rahmonov 1987; Ibadinov 1989; Kochan et al. 1989; Spohn et al. 1989; see also Grün et al.'s paper in these proceedings). The literature is now so extensive that even this long list of papers represents, in fact, only a selection of all the relevant publications.

\subsection{HYDRODYNAMIC MODELS OF JETS}

In the past several years, significant progress has been reported in the understanding of the outflow mechanism and the thermophysical properties of gas and dust emissions expanding from a comet nucleus in a highly anisotropic fashion. Vergazov and Krasnobaev (1985) presented a model of an axisymmetric flow of pure water vapor with an expansion-velocity distribution fairly similar to that of a spherically symmetric flow, but with a dramatic effect on the $\mathrm{OH}$ brightness distribution in the coma. Observations of several comets, including Halley and Encke, show that both the radio spectra of $\mathrm{OH}$ at $1667 \mathrm{MHz}$ (cf. the relevant references in Sec. 2.2) and the UV spectra of OH at $3085 \AA$ (A'Hearn and Schleicher 1988) exhibit significant asymmetries in the line-of-sight velocity profile.

Kömle and Ip (1987a) formulated an axisymmetric hydrodynamic model to calculate the dynamical solutions for an anisotropic flow of pure gas, applying it to two idealized scenarios: (1) a strong source, $10^{\circ}$ across, centered on the subsolar point and superimposed 
on a much weaker ambient emission field, and (2) a ringlike source, $5^{\circ}$ wide, centered on the subsolar point at an angular distance of $25^{\circ}$ and with no activity inside the ring. Kömle and Ip calculated the quasi-steady state water flow regime for both scenarios and also transient flow conditions for the first scenario at the emission's initiation and termination times. They found that in the case of the central source, a strong lateral flow develops, causing the expanding jet to acquire, several kilometers above the nucleus surface, the outlines of an expanding cone, in which the density peak is shifting-with a gradually decreasing lateral velocity - away from the subsolar point, eventually making an angle of $40^{\circ}-45^{\circ}$ with this direction. In the case of the ringlike source, the flow characteristics are quite different: the primary density peak rapidly develops near the subsolar point (implying a strong flow toward the central "hole") and there is an inconspicuous secondary maximum about $60^{\circ}$ from the primary peak. Kömle and Ip's investigation into the time-dependent behavior following the initiation and termination of the activity suggests that a typical time scale of a few minutes is needed to achieve steady state. In their follow-up paper, Kömle and Ip (1987b) studied effects on the density profiles of the infrared radiation cooling (Shimizu 1976; Crovisier 1984) and the gas heating by the ejected dust (Marconi and Mendis 1986), introduced additional variations to their initial conditions for the anisotropy, and compared the resulting sunward/tailward asymmetry in the column density of water calculated from their models with the results of Weaver et al.'s (1986) observations of Halley's comet.

Kitamura's (1986) first hydrodynamic models for axisymmetric jets already discriminated between pure gas and dusty gas scenarios. He assumed that water is the only gas species and that the emission in the jet is superimposed on a constant background gas flux. In the special case of a narrow, pure gas jet, Kitamura's results are in many respects similar to those by Kömle and Ip for the $10^{\circ}$ cone angle. However, for a gas jet three times as broad, Kitamura's results indicate that the central density peak is preserved to larger distances from the nucleus. For a narrow dusty gas jet, the lateral expansion of dust particles to the wings of the cone follows closely that of the gas, some $35^{\circ}$ away from the normal to the source, but this effect is confined only to the innermost region of the coma. The dust-density peaks at $35^{\circ}$ survive even at larger distances from the nucleus. Kitamura found that the overall character of the emission pattern is relatively independent of the gas viscosity, the assumed profile of the ejecta at the surface, and the particle size employed (at least within the limits of 0.4 to $1.5 \mu \mathrm{m}$ ), even though the gas-dust interaction increases with decreasing grain dimension.

In his second paper, Kitamura (1987) considered the cases of isolated axisymmetric pure and dusty gas jets, that is, he assumed that there is no gas emission from the nucleus outside the discrete source. Of particular interest are his results for the dusty gas jets, calculated for an inviscid flow of $\mathrm{H}_{2} \mathrm{O}$ (slip-wall conditions). The peak densities of both the gas and the dust follow the initial direction of emission, with no signs of any side enhancement, because the flow from the discrete source can expand freely in the lateral directions without being decelerated by the ambient gas flow. For the same reason, some of the gas streams along the mantled regions of the nucleus as it tends to equalize the pressure across and around the nucleus. Large numbers of submicron dust grains, which are strongly coupled with the gas, were shown by Kitamura to be entrained in a surface breeze over the mantled regions of the nucleus (cf. Keller et al. 1987 for observational evidence of this breeze on the Giotto images of Halley's nucleus). By contrast, the cloud of micron-sized and larger particles expands primarily in the radial direction, thus producing a collimated flow diagnostic of the initial emission vector. 
From the standpoint of modelling the gross dust-coma morphology on ground-based images of comets, Kitamura's hydrodynamic treatment of isolated dusty gas jets shows that dust particles in jets cannot be smaller than a few tenths of a micron. Interestingly, this limit virtually coincides with the critical size of grains that get charged in the solar radiation field to electrostatic potentials sufficiently high that the grains' motions are strongly affected by the magnetic fields (e.g., Horányi and Mendis 1985, 1986) and become essentially unpredictable in the complex plasma environment of the coma and tail. On the other hand, when decoupled from the gas flow, the larger dust particles in the collimated cone of a jet have motions that become controlled by solar gravity and solar radiation pressure and are thus easily predictable. The fact that dust grains with randomized motions happen to be much smaller than the wavelength of visible light and therefore nearly transparent, while particles with highly organized motions are optically efficient, makes it possible to detect jet features on ground-based images of comets, even though a minor contribution to the dust-coma brightness from the tiniest grains and imperfect collimation of the flow of larger ejecta from the discrete sources do bring about a certain degree of scatter in the particle velocity field in the coma that decreases the contrast of the observed structures.

\section{Coma Morphology and Discrete Active Regions on a Rotating Nucleus}

The understanding of the formation and evolution of comets depends critically upon our perception of the nature and characteristics of comet activity. In this context, the concept of localized active regions represents a new way of thinking that contrasts with the traditional view of nearly uniform outgassing from much of the nucleus surface. Direct imaging of these regions on the nucleus of Halley's comet with the cameras on board the intercepting spacecraft (cf. Szegö's paper in these proceedings; also see, e.g., Keller et al. 1987) illustrates the overwhelming observational support for the new concept, whose recent, long-overdue incorporation into the physical models of comets exemplifies the profound role this concept has begun to play in cometary science of the post-Halley era.

\subsection{PRIMARY OBJECTIVES OF COMA-MORPHOLOGY STUDIES}

The primary objectives of morphological studies of the dust coma include the understanding of the initiation, evolution, and termination of activity from the regions that produce the dust features; the physical properties and chemical composition of the active regions and their ejecta; the source function of dust and its temporal variations; the extent of the regions, their possible migration over the nucleus surface, and the nature of their interaction with the inert mantle; the processes that may cause the regions to become dormant, rejuvenated, or extinct; and the effects of the individual regions on the evolution of the nucleus as a whole. While it may require several rendezvous and nucleus-sample return missions to settle these issues, some of the aspects can be addressed by synergistic investigations of the near-nucleus environment that include the types of approach described in this review.

The unique property of comet activity is its interaction with the nucleus rotation. Two types of changes that are generally experienced by any surface element on a spinning nucleus are diurnal and seasonal variations. The seasonal effects bring about the perihelion asymmetry of the gas- and dust-production curves, which is now extremely well documented for comet Halley as well as many other comets. The diurnal effects cause short-term variations in outgassing, but the spin modulation of the activity is at least partially masked by the 
extended residence time of dust ejecta in the coma, which is often comparable with, or longer than, the rotation period. If a comet's rotational motion is far from pure spin, the discrimination between the seasonal and diurnal variations may become difficult and, in an extreme scenario, their definitions become meaningless. Since the momentum transferred by outgassing to the nucleus is sufficient both to alter slightly the comet's orbital motion (nongravitational perturbations; Sec. 5.5) and to exert torques on its polar axis (forced precession), not only does rotation affect activity, but, conversely, rotation is affected by activity. Thus, modelling of the comet's surface morphology provides, as a byproduct, information on the object's rotation properties. The experience with Halley's comet suggests that free precession of the nucleus is involved and that analysis of temporal variations in brightness (Millis and Schleicher 1986, and many others) identifies a sequence of emission episodes that is controlled by general rotation and not by the spin rate alone.

A particularly intriguing aspect of the interaction between comet rotation and activity is a possible long-term effect on both the rotation stability and the distribution of active regions on the nucleus surface. Very little is known about potential patterns in evolutionary paths for cometary nuclei, but one of the major issues in this category is the nature and significance of the basic shape of the nucleus (Sec. 5.1). Is the elongated figure of some nuclei primordial or has it been acquired in the process of outgassing and dust emission? Are there "preferred" locations for active regions on the nucleus surface and, if so, do they depend upon the nuclear shape and/or the tilt of the angular momentum vector with respect to the comet's orbital plane? For example, is the occurrence of high-latitude sources statistically correlated with a high obliquity (a high inclination of the equatorial plane to the orbital plane)? Is the high-obliquity configuration more stable dynamically than the low-obliquity configuration? Definitive answers to these fundamental questions of cometary physics will not be known for some time to come.

\subsection{COMPUTER SIMULATION OF DUST-COMA MORPHOLOGY}

In the morphological investigations of dust comets, the next step was to address the issue of great diversity among individual objects: Why do some of them (such as Halley) display spiral jets and quasi-parabolic envelopes, while others exhibit nearly structureless fans or semi-circular halos or yet another type of feature; and why do so many comets show features only rarely or not at all? Also, why do some comets display more than one type of structure, whether simultaneously or at different times?

Any aspiring morphological concept should be able to simulate all the basic types of the observed features in a uniform manner. This requisite necessitated a revision (Sekanina 1987b) of an earlier paradigm for fanlike comets (Sekanina 1979), making it compatible with the Halley model, and led to an investigation of structural diversity that can be simulated by varying the parameters of synthetic, computer-generated images.

In the idealized case of a pointlike emission source located in the nucleus equatorial plane, which coincides with the comet's orbit plane, the cometocentric motions of dust particles ejected normal to the surface (that is, under conditions of perfect collimation) with a particular velocity $v$ and subjected to a constant acceleration $g$ by solar radiation pressure are described by the equations

$$
\begin{aligned}
& x(t, \theta)=f(t, \theta)\left[v \cos \theta-\frac{1}{2} g f(t, \theta)\right], \\
& y(t, \theta)=f(t, \theta) v \sin \theta
\end{aligned}
$$


where

$$
f(t, \theta) \doteq\left(t-t_{b}\right)-\frac{P}{2 \pi}\left(\theta-\theta_{b}\right)
$$

$x$ and $y$ are rectangular coordinates of the inertial system at time $t$ oriented, respectively, to the Sun and $90^{\circ}$ ahead of it in the direction of rotation; $P$ is the spin period; $\theta$ is the angle of ejection, reckoned from the sunward direction in the sense of rotation and measured in radians; and $t_{b}$ and $\theta_{b}$ are the time and ejection angle at the beginning of emission. Equations (1) and (2) hold for any time $t \geq t_{b}$, while the function $f(t, \theta)$ is here defined for emission during one rotation, that is, for $\theta_{b} \leq \theta \leq \theta_{f}$, where $\theta_{f}$ is the ejection angle at the time of emission termination.

The situation with actual dust features is unfortunately much more complex than in the described idealized case, because a comet's spin axis is not, as a rule, normal to its orbit plane and its position may vary with time; several regions are sometimes active at the same time, they are usually not located on the equator, and they may not always be approximated by point sources; emission may proceed over many rotations; and, most certainly, dust particles are not all ejected with the same velocity and subjected to the same radiation-pressure acceleration. In addition, particle acceleration varies with heliocentric distance-an effect that especially near the Sun becomes significant soon after ejection. Also, Earth's cometocentric position is seldom near the direction of the comet's spin axis, so that features projecting onto the plane of the sky are foreshortened by some unknown amount. As a result of all these complications, the positional analysis of actual dust features is considerably more involved than indicated by Eqs. (1) and (2) and it is essential that the rigorous equations of dust-particle motion be employed in the computer code. It will be shown in Sec. 3.3 that of particular concern in practice is the problem of multiple solutions, whose elimination demands that coma morphology always be analyzed as a function of time. This requires a sufficiently dense temporal coverage by high-resolution near-nucleus images to provide adequate information on the jets' evolution.

Retaining only the constraints of a fixed spin-axis position and perfect collimation of the ejecta (but allowing some randomization of their ejection velocities and radiation-pressure accelerations), the effects of varying the computer model's parameters on the appearance of the resulting synthetic images were investigated next. The results of the initial simulations showed conclusively (Sekanina 1987b) that the very existence of discrete emission sources on a rotating cometary nucleus produces grossly diverse patterns in the dust coma even under the conditions of a fixed Sun-Earth-comet geometry. The coma outlines on the simulated images depend strongly upon the spin-axis orientation with respect to both the Sun and Earth, the rotation period, the surface distribution of the active regions (the source function), their extent and mode of outgassing, the local topography, and the dynamical constants of the ejecta's particle-mass distribution. It was shown that certain combinations of the source function and the spin-axis position lead to diagnostic dust-coma patterns that can straightforwardly be identified. For example, a persisting sunward fan-shaped coma is indicative of outgassing, in a high-obliquity configuration (the spin axis is near the comet's orbital plane), from regions that are relatively near the sunlit rotation pole. If the nucleus is in pure spin, activity can essentially be continuous for a number of weeks or even months and the local erosion rate is substantially higher than in a low-obliquity configuration (the spin axis is nearly perpendicular to the orbital plane), which is associated with the day/night insolation regime and characterized by more pronounced diurnal variations. 
On the other hand, the presence in the coma of prominent corkscrewlike jets or expanding halos is often diagnostic of the rotation axis that points approximately in the direction of Earth. Low-obliquity configurations usually produce Halley-type spiral jets that unwind from the nucleus condensation and, on the time scales of a day or so, turn into envelopes on the sunward side of the coma (Sec. 2.2).

\subsection{COMPARISON OF OBSERVED AND SYNTHETIC IMAGES OF DUST COMETS}

Besides its capability to generate synthetic images that-based on a set of reference parameters appropriate for the given comet at the given times-match the observed dust features in the coma, the computer model discussed in Sec. 3.2 can often yield images that-based on entirely fictitious parameters-successfully imitate features observed in other comets at other times. To illustrate this point, the reader is referred to Fig. 1, which displays images of six comets (three drawings based on visual telescopic observations and three photographs, one of them image-processed) and six computer-simulated images. Each of the latter was generated for periodic comet Tempel 2-which is not among the six comets-at an arbitrarily chosen time of 44 days following its perihelion passage in 1920 . Comet Tempel 2, which is known to retain the same general appearance over the period of several months after perihelion, exhibits a distinct fan-shaped coma on the plates exposed by $W$. Baade with the $100-\mathrm{cm} \mathrm{f} / 3$ reflector at the Hamburger Sternwarte about nine weeks after the 1920 perihelion (cf. Fig. 6 of Sekanina 1987c for these digitally processed images); also, at the same apparition, the fan was detected visually and consistently reported as a sunward tail by Chofardet (1923) 41 days after perihelion and by Van Biesbroeck (1921) 6 days later. The appearance of comet Tempel 2 at the time is unquestionably approximated most closely by the image of Encke's comet in Fig. 1, except that the fan's axis deviated by $20^{\circ}-30^{\circ}$ more from the Sun's direction.

Table 1 offers the parameters for each synthetic image in Fig. 1. The columns list the image number, the constants $\Phi$ and $I$ of the spin-axis position (cf. Sekanina 1981a for their definitions), the angles the spin axis subtends with the directions of the Sun and Earth (an angle smaller than $90^{\circ}$ means that the northern pole is turned toward the Sun or Earth), the rotation period, the latitude of the active region and its angular distance from the subsolar meridian at the chosen time of $\mathbf{4 4}$ days past perihelion (a negative angle means the morning; a positive angle, the afternoon), the duration of the modelled dust emission (whether continuous or with interruptions), and the peak acceleration by solar radiation pressure, $\boldsymbol{\beta}_{\max }$ (in units of solar attraction), exerted on the ejecta.

Table 1 shows that the morphological diversity in Fig. 1 is imitated with only two values of the rotation period and, in four out of the six cases, with a single pointlike active region. The models are further constrained by employing a common parametric function that expresses the dependence of particle ejection velocity on solar radiation pressure, $v(\beta)$, assigning velocities of $25 \mathrm{~m} / \mathrm{s}$ to particles with $\beta=0.001,200 \mathrm{~m} / \mathrm{s}$ to particles with $\beta=0.1$, and $510 \mathrm{~m} / \mathrm{s}$ to particles with $\beta=2.5$. The correspondence between the observed and computersimulated images indicates that if one were able to change appropriately the morphological and rotation properties of comet Tempel 2, its dust coma could resemble that of any of the six comets.

Space allows pointing out only some of the interesting properties of the synthetic images and their diagnostic features. In Images \#1, \#4, and \#6, an important factor is the small angular distance between the spin axis and Earth's direction. The primary distinction 


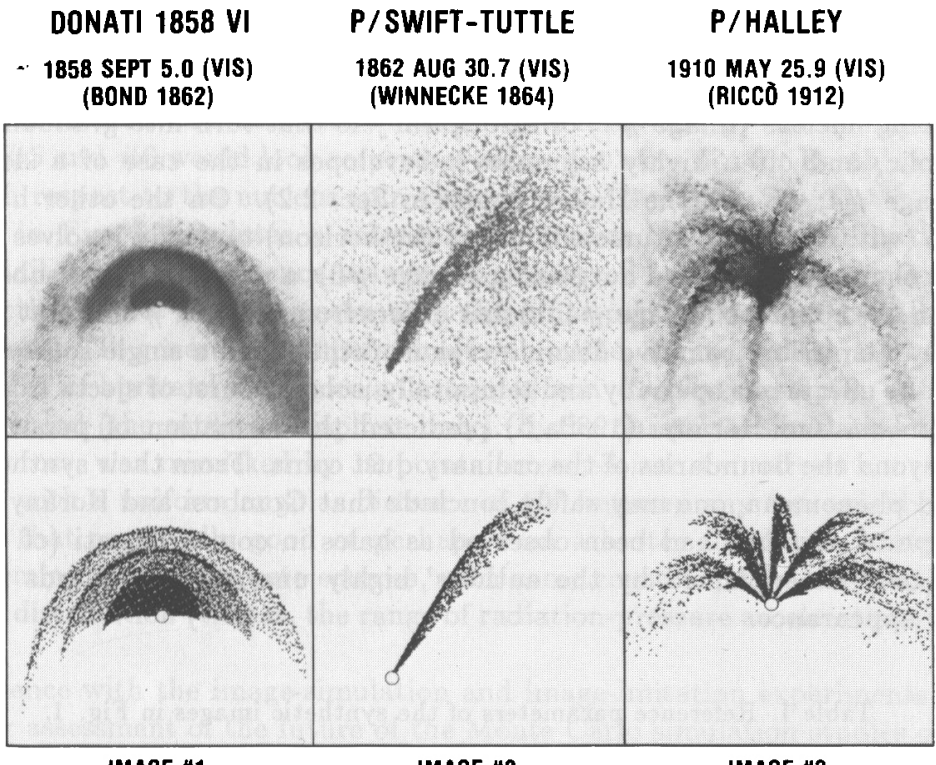

IMAGE \#1

IMAGE \#2

IMAGE \#3

BENNETT 1970 II P/ENCKE

1970 MAR 28.5 (PHOT, IP) 1980 OCT 10.5 (PHOT) (LARSON \& MINTON 1972; LARSON 1986)

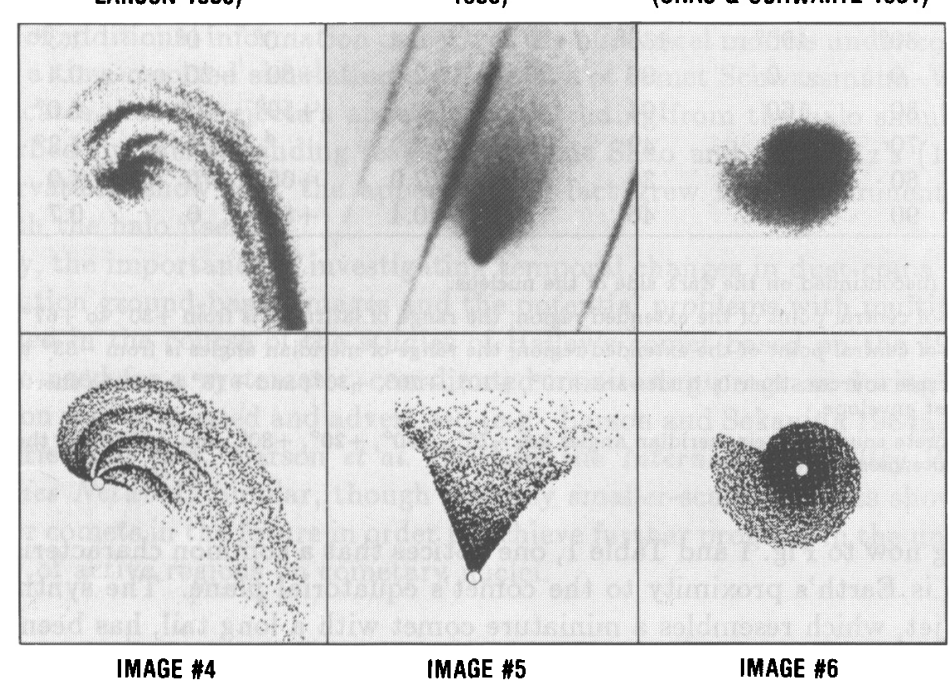

IMAGE \#4
(DJORGOVSKI \& SPINRAD 1985)

\section{P/SCHWASSMANN-} WACHMANN 1

1981 FEB 10.3 (PHOT) (SHAO \& SCHWARTZ 1981)

Figure 1. Coma morphology of six dust comets (top rows) and its imitation by synthetic, computergenerated images (lower rows). The observation times are in UT. A drawing is identified by VIS, a photograph by PHOT, and a digitally processed image by IP. The synthetic images, calculated for comet Tempel 2, are marked \#1 through \#6. The Sun is always at the top, but the image scales are not normalized. (Original photographs courtesy of S.M. Larson, H. Spinrad, and C.-Y. Shao.) 
between Images \#1 and \#4 on the one hand and \#6 on the other is the latitude difference of the active regions: their near-equatorial locations dictate an on-and-off diurnal cycle of activity, which organizes the dust ejecta into concentric halos on the sunward side of a rapidly rotating nucleus (Image \#1) or into spiral jets that turn into gradually expanding, quasi-parabolic, and often highly asymmetric envelopes in the case of a slowly rotating nucleus (Image \#4; cf. also the Halley model in Sec. 2.2). On the other hand, a polarday scenario (with the Sun continuously above the horizon) typically involves high-latitude sources with their uninterrupted outgassing (Image \#6), a pattern that is substantially less dependent on the spin rate. Image \#4 further differs from \#1 and \#6 in that it is modelled as a product of emission from five discrete regions rather than a single source.

Studying the effects of a spatially and temporally isolated burst of ejecta from a cometary nucleus, Gombosi and Horányi $(1986 \mathrm{a}, \mathrm{b})$ predicted the formation of persistent sunward envelopes beyond the boundaries of the ordinary dust coma. From their synthetic images of the proposed phenomena, one may safely conclude that Gombosi and Horanyi's suggestion that this type of envelope had been observed as halos in comet Donati (cf. Image \#1 in Fig. 1) can only be explained by the authors' highly uncritical standards for judging a similarity of appearance.

Table 1. Reference parameters of the synthetic images in Fig. 1.

\begin{tabular}{|c|c|c|c|c|c|c|c|c|c|}
\hline \multirow{2}{*}{ Image } & \multicolumn{2}{|c|}{$\begin{array}{c}\text { Spin axis } \\
\text { orientation }\end{array}$} & \multicolumn{2}{|c|}{$\begin{array}{l}\text { Angle between } \\
\text { spin axis and }\end{array}$} & \multirow{2}{*}{$\begin{array}{c}\text { Rota- } \\
\text { tion } \\
\text { period } \\
P \\
\text { (days) }\end{array}$} & \multicolumn{2}{|c|}{$\begin{array}{l}\text { Location of } \\
\text { dust source }\end{array}$} & \multirow{2}{*}{$\begin{array}{l}\text { Activ- } \\
\text { ity } \\
\text { dura- } \\
\text { tion } \\
\text { (days) }\end{array}$} & \multirow{2}{*}{$\begin{array}{c}\text { Peak } \\
\text { accel- } \\
\text { era- } \\
\text { tion } \\
\beta_{\text {max }}\end{array}$} \\
\hline & $\begin{array}{l}\text { Obliq- } \\
\text { uity I }\end{array}$ & $\begin{array}{c}\text { Argu- } \\
\text { ment } \Phi\end{array}$ & Sun & Earth & & $\begin{array}{l}\text { Lati- } \\
\text { tude }\end{array}$ & $\begin{array}{c}\text { Merid- } \\
\text { ian }\end{array}$ & & \\
\hline 1 & $80^{\circ}$ & $190^{\circ}$ & $136^{\circ}$ & $170^{\circ}$ & 0.4 & $0^{\circ}$ & $0^{\circ}$ & $1.3^{a}$ & 2.5 \\
\hline 2 & 0 & 0 & 90 & 90 & 2.0 & +30 & -20 & 0.4 & 1.1 \\
\hline 3 & 80 & 160 & 104 & 149 & 0.4 & $+50^{b}$ & $0^{c}$ & $2.0^{a}$ & 2.5 \\
\hline 4 & 70 & 10 & 49 & 20 & 2.0 & $d$ & $e$ & $1.2^{a}$ & 2.5 \\
\hline 5 & 80 & 90 & 36 & 81 & 2.0 & +65 & -70 & 4.9 & 0.2 \\
\hline 6 & 90 & 10 & 46 & 1 & 0.4 & +50 & 0 & 0.7 & 0.1 \\
\hline
\end{tabular}

a Activity discontinued on the dark side of the nucleus.

${ }^{b}$ Position of central point of the extended region; the range of latitudes is from $+30^{\circ}$ to $+67^{\circ}$.

$c$ Position of central point of the extended region; the range of meridian angles is from $-33^{\circ}$ to $+33^{\circ}$.

$d$ Five discrete sources: their latitudes are $+10^{\circ},+10^{\circ},+20^{\circ},+30^{\circ}$, and $+10^{\circ}$ from the shortest jet to the outermost envelope.

$e$ Five discrete sources: their meridian angles are $-70^{\circ},-30^{\circ},+20^{\circ},+80^{\circ}$, and $+90^{\circ}$ from the shortest jet to the outermost envelope.

Returning now to Fig. 1 and Table 1, one notices that a common characteristic of Images \#2 and \#5 is Earth's proximity to the comet's equatorial plane. The synthetic image of the slender jet, which resembles a miniature comet with a long tail, has been generated as a continuous flow of dust particles, in an almost edge-on projection onto the plane of the sky, ejected since sunrise from a source at a moderate latitude. The jet's deviation from the Sun's direction is, in this case, a measure of the source's angular distance from the equatorial plane. On the other hand, Image \#5 is a simulation of the typical fan-shaped coma, a rather frequently observed phenomenon. The parameters used to generate the 
image confirm the diagnostic nature of this type of feature, which was briefly described in Sec. 3.2: it is, indeed, characteristic of the near- $90^{\circ}$ tilt of the comet's equatorial plane to the orbit plane, with the source at a high latitude of the predominantly sunlit hemisphere and with Earth located outside of the emission cone described by the spinning source. In fact, Images \#5 and \#6 would look somewhat alike, if it were not for Earth's very different positions with respect to the nucleus spin vector.

Image \#3 in Fig. 1 simulates a relatively complicated morphological pattern. Even though in detail the correspondence between the observed and the synthetic images in this pair is distinctly inferior in comparison with the other pairs, the general outlines do show some resemblance. To generate the "palm-tree" silhouette, the individual jets were modelled as products of brief bursts of dust from an extended active region, assuming one burst every 0.1 rotation period (the sunlit hemisphere only). In the numerical simulation the extended region, $45^{\circ}$ across, was mimicked by 12 points arranged into two circles concentric about a 13th point, with a uniform angular distance of $12^{\circ}$ between any two neighboring points. It is possible that an equally good or perhaps even a better simulation pattern could be achieved assuming several discrete sources, with some constraints on their activity and/or particle mass distribution (that is, the range of radiation-pressure accelerations and ejection velocities).

The experience with the image-simulation and image-imitation experiments achieved so far is vital for assessment of the future of the Monte Carlo simulation studies of dust-coma morphology. The good news is that the technique applied, even though trial-and-error in nature, is sufficiently powerful to match extremely diverse patterns with a very restricted set of reference parameters. The bad news is the obvious existence of multiple solutions, which-unless properly discriminated and prioritized as to their physical accuracy-would render the whole approach effectively useless. Luckily, temporal morphological variations in the dust coma represent a discriminating factor of enormous diagnostic value, providing a large amount of additional information on each of the numerical models under consideration. For example, a time-resolved simulation of the image of comet Schwassmann-Wachmann 1 in Fig. 1 indicates that the ejecta's appendage protruding from the halo should gradually become absorbed by this expanding feature, whereas Shao and Schwartz's (1981) photographic observations show that the appendage in fact grew more prominent with time, compared with the halo itself.

Fortunately, the importance of investigating temporal changes in dust-coma morphology on high-resolution ground-based images and the potential problems with multiple solutions were recognized in the course of the studies of Halley's comet based on the 1910 observations, and the need for a systematic, coordinated monitoring program during the comet's 1986 apparition was advocated and advertised (e.g., Larson and Sekanina 1984a,b), and subsequently carried out (e.g., Larson et al. 1987) by the International Halley Watch's Near Nucleus Studies Network. Similar, though possibly smaller-scale, projects should be organized for other comets in the future in order to achieve further progress in the understanding of the history of active regions on cometary nuclei.

\section{Temporal Variations in Gas Production From Discrete Active Regions}

In conformity with the existing terminology, a comet's production rate for any gaseous species at a given time is defined as the number of molecules (or atoms) released by the comet 
from its whole nucleus surface per unit time (usually per second). The mass production rate is the product of the mass of a molecule/atom of the species and the production rate and is expressed in $\mathrm{g} / \mathrm{s}$ or $\mathrm{kg} / \mathrm{s}$. The mass production rate can also be calculated for dust ejecta, if their particle-mass distribution function is known or assumed. The sublimation rate of a gas species is defined as the number of molecules/atoms released from a unit surface area (usually $1 \mathrm{~cm}^{2}$ or $1 \mathrm{~m}^{2}$ ) of the nucleus surface per unit time and it is calculated from a sublimation theory applied to the expected physical conditions on the nucleus; it is therefore model-dependent and a function of both time and location. If the sublimation rate does not vary significantly over the active region, or if the average sublimation rate is known or can be estimated, the production rate is given as the product of this sublimation rate and the surface area involved.

\subsection{THE PRODUCTION, SUBLIMATION, AND LIGHT CURVES}

Arguments based on both observational (e.g., Huebner et al. 1986; Reitsema et al. 1989) and theoretical (Kitamura 1987; cf. Sec. 2.3) evidence indicate that the discrete active regions on Halley's nucleus imaged by the Giotto camera represented the primary source for both dust and gas production and that the mantled areas either did not outgas at all or contributed, at best, only a minor fraction of the comet's total gas output at the time. Huebner et al. showed that the observed gas production rate was indeed reasonably compatible with the theoretical sublimation rates, calculated from a simple energy-balance equation, multiplied by the estimated surface area of the imaged active regions. If-as seems likely-outgassing from discrete sources dominates the activity of many comets, then studies of their gas production are essentially reduced to studies of their active regions. Insolation and sublimation patterns of anisotropically outgassing comets were studied by Cowan and A'Hearn (1979), Sekanina (1979), and others.

Temporal variations in a comet's production rate determine its production curve and one can similarly define a sublimation curve. These may or may not correlate with the light curve, which is defined by temporal variations in the comet's brightness normalized to $1 \mathrm{AU}$ from Earth. The physical meaning of a light curve depends upon the instrument, the detector, and the filter employed (continuum, emission-band, broadband; visual, photographic, photoelectric, CCD, infrared; nuclear, integrated, eye estimate, etc.). Under ideal conditions of uniform gas and dust emission, one could expect a high degree of correlation among the diverse production and light curves for a given comet. Unfortunately, departures of any kind from emission uniformity tend to destroy these correlations and introduce uncertainties in the various mass-loss characteristics of comets. Two examples illustrating the range of scenarios are comets Halley and Encke. For Halley, available information suggests that the production rate of water and the normalized integrated visual brightness correlate fairly well both before and after perihelion, indicating that the brightness varies, on the average, as a 1.6 power of the water-production rate (Sekanina 1989). For Encke's comet, on the other hand, A'Hearn et al. (1985) concluded that the visual light curve is consistent with the production curves for $\mathrm{C}_{2}$ and $\mathrm{CN}$ but not $\mathrm{OH}$ (and therefore $\mathrm{H}_{2} \mathrm{O}$ ) and that the two groups of curves exhibit perihelion asymmetries of opposite sense (Sec. 4.3).

The traditional tacit assumption of an essentially symmetric light curve (peaking at perihelion) applied to predict a comet's brightness over extended periods of time is known to have been responsible for numerous fiascos in the past. One of the most memorable instances was comet Kohoutek 1973 XII, whose disappointing postperihelion performance was contrary to expectations based on such predictions. 


\subsection{MODELLING WATER-PRODUCTION CURVES OF COMETS WITH ACTIVE REGIONS}

The seasonal changes that active regions experience on a rotating nucleus (Sec. 3.1) rule out symmetric light or production curves except under special circumstances. In order for the reader to appreciate the variety of scenarios possible for comets with morphologically heterogeneous nuclei, Figs. 2-4 show examples of water sublimation curves calculated for a spherical nucleus in an unperturbed heliocentric orbit with a perihelion distance of $1.5 \mathrm{AU}$, an eccentricity of 0.58 , and a revolution period of $6.75 \mathrm{yr}$, typical for many short-period comets of Jupiter's family. The rotation-averaged sublimation rates of pointlike sources at various locations on the nucleus surface are calculated as a function of time, employing the technique described in Sekanina (1988b). In all the cases displayed, the spin axis is assumed to be inertially fixed in the comet's orbit plane (obliquity $90^{\circ}$ ). The axial position and the locus described by the active region during rotation are shown schematically to the right of each sublimation curve in an orbital coordinate system (in two projections, onto the orbit plane and normal to it), where $\mathbf{P}$ is the Sun-comet vector at perihelion, $\mathbf{Q}$ the vector pointing $90^{\circ}$ ahead of $\mathbf{P}$ in the orbit plane in the direction of the orbital motion, and $\mathbf{R}$ the vector directed to the northern orbital pole. The rotation parameters $\Phi$ and $I$ are for each configuration listed in the captions. Three symmetric sublimation curves are shown in Fig. 2: the symmetry is seen to be a result of the spin axis pointing at the Sun at perihelion in panels $a$ and $b$, while in panel $c$ the symmetry follows from the fact that the spin axis is normal to the Sun's direction at perihelion and the active region is located on the equator. Comparison of panels $a$ and $b$ shows that for the same orientation of the spin axis, the sublimation curve depends critically upon the latitude of the active region: the difference of $30^{\circ}$ turns the curve with a broad maximum at perihelion in panel $a$ into a double-peaked curve with a deep minimum at perihelion in panel $b$, a change that is caused by the dramatic drop in the insolation rate at the location of the source near perihelion. On the other hand, panel $c$ illustrates that an equatorial active region can also display a steep curve, sharply peaked at perihelion, if the spin axis is turned in the orbit plane by $90^{\circ}$ : appreciable activity then proceeds only during a time interval of 300 days centered on the perihelion point. Theoretically, a secondary period of activity, symmetric with respect to aphelion, takes place at larger heliocentric distances (more than 250 days from perihelion).

More typical, asymmetric sublimation curves are displayed in Figs. 3 and 4. Panel $a$ of Fig. 3 shows that when an active region is at a moderate latitude, the production is greater along the inbound or outbound leg of the orbit (with the peak shifted correspondingly), depending upon whether the spin axis points at the Sun a little before or after perihelion. A peculiar curve with two uneven peaks and a deep minimum shifted to either side of perihelion is shown in the figure's panel $b$ to result for the same spin-axis position but with the active region located on the equator. The even more asymmetric sublimation curves, presented in Fig. 4, formally simulate two phenomena that are commonly described by observers as, respectively, an outburst and a disappearance of a comet. As shown, the precipitous increase in outgassing can be interpreted as a result of an active region's emersion from the polar night (during which the Sun is permanently below the horizon), while its immersion into the polar night brings about rapid deactivation. The highly asymmetric curves are generally associated with active regions at large angular distances from the equatorial plane and they can peak either before or after perihelion, depending primarily upon the rotation-pole orientation (cf. panels $a$ and $b$ in Fig. 4). Examples of potential applications of the described model are discussed in some detail in Secs. 4.3-4.5. 
WATER SUBLIMATION FROM ACTIVE REGION ON COMETARY NUCLEUS

$(q=1.5 \mathrm{AU}, \mathrm{e}=0.58)$

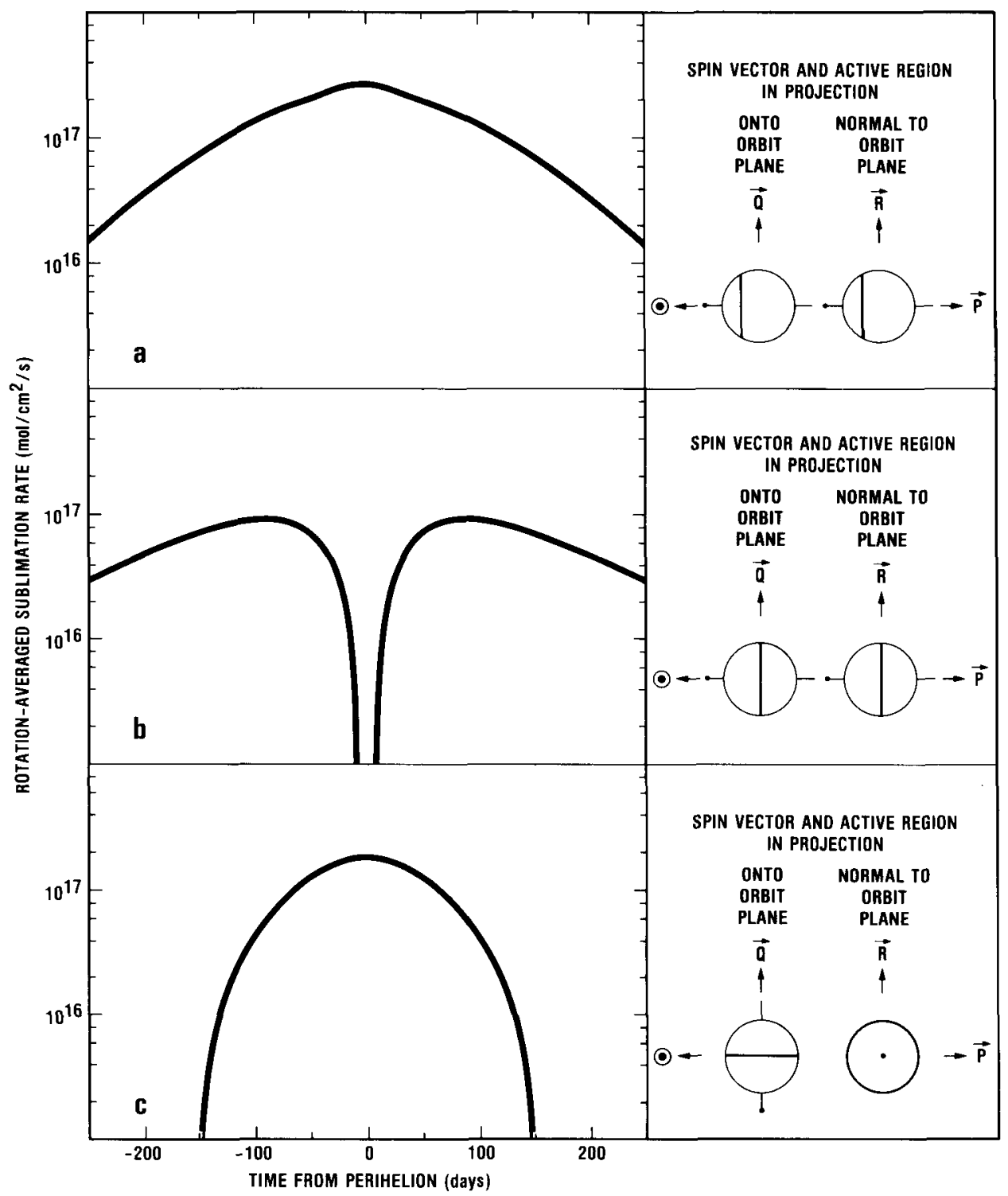

Figure 2. Calculated rotation-averaged sublimation curves for the nuclei of comets with a single active region on the surface. The spin-axis orientation and the locus described by the active region during rotation are shown schematically to the right of each sublimation curve in an orbital coordinate system, identified by the standard vectors $\mathbf{P}, \mathbf{Q}$, and $\mathbf{R}$ (cf. text). The two rotation parameters (the argument $\Phi$ and the obliquity $I$ ) and the active region's latitude $\phi$ are: $90^{\circ}, 90^{\circ},+30^{\circ}$ for panel $a ; 90^{\circ}, 90^{\circ}, 0^{\circ}$ for panel $b$; and $0^{\circ}, 90^{\circ}, 0^{\circ}$ for panel $c$. The northern pole is identified with a dot on the spin axis and the Sun's direction is indicated by a circled dot (in the direction of $-\mathbf{P}$ ). 
WATER SUBLIMATION FROM ACTIVE REGION ON COMETARY NUCLEUS

$(q=1.5 \mathrm{AU}, \mathrm{e}=0.58)$

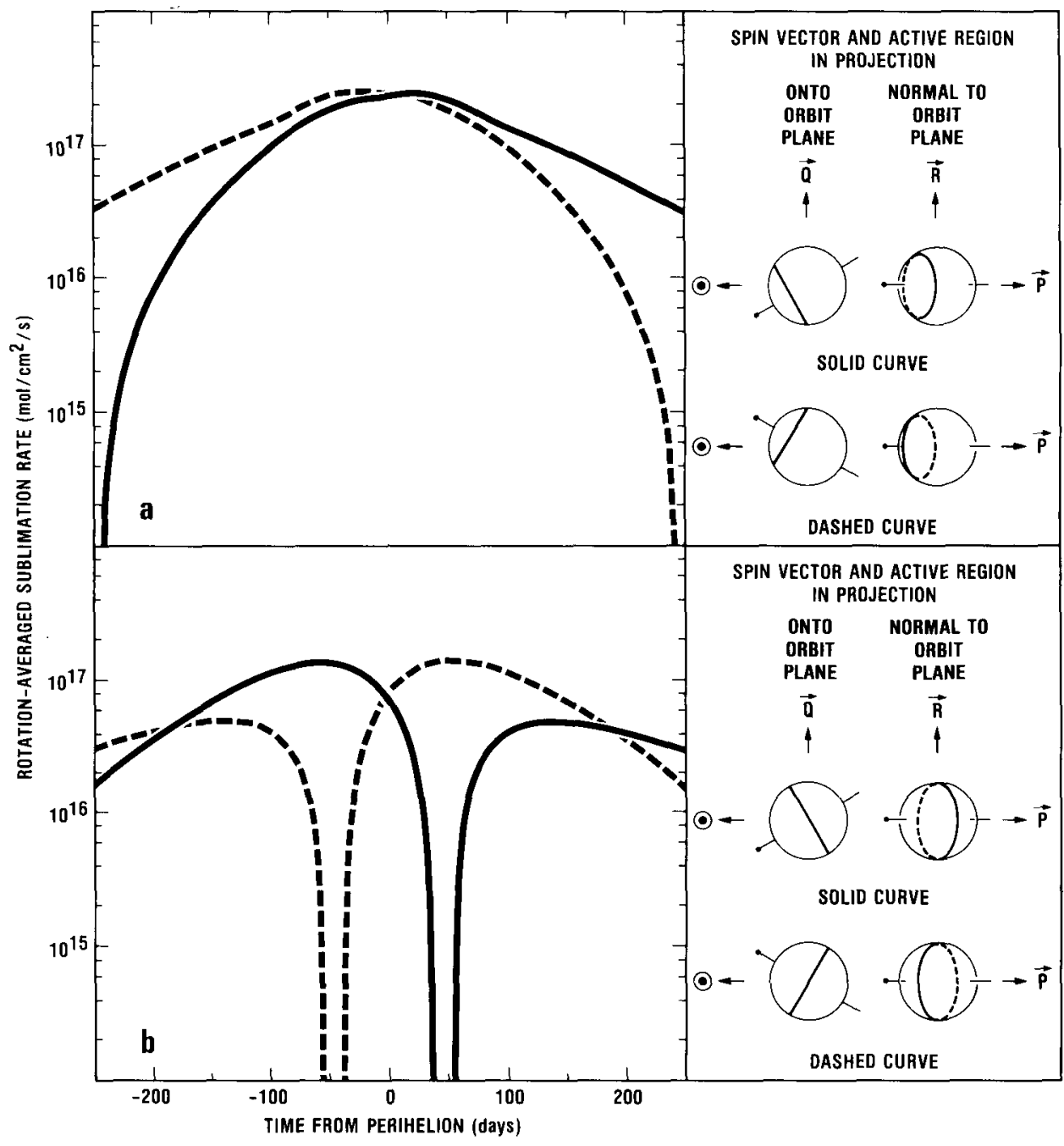

Figure 3. Calculated rotation-averaged sublimation curves for the nuclei of comets with a single active region on the surface. The parameters $\Phi, I$, and $\phi$ for the solid/dashed curves are: $60^{\circ} / 120^{\circ}$, $90^{\circ},+30^{\circ}$ for panel $a$ and $60^{\circ} / 120^{\circ}, 90^{\circ}, 0^{\circ}$ for panel $b$. See the caption to Fig. 2 for further information.

\subsection{PERIODIC COMET ENCKE}

Observed at more than 50 returns to the Sun, Encke's comet is one of the best studied short-period comets and the only one for which the conditions required for modelling the production curve are satisfied: (1) the water-production rates are available over a fairly 
long arc of the orbit; (2) outgassing from the discrete sources on its nucleus is established with a fair degree of confidence; and (3) the spin vector and the source distribution function are sufficiently well known. (Halley's comet will qualify, when the problem of its rotation is fully resolved.)

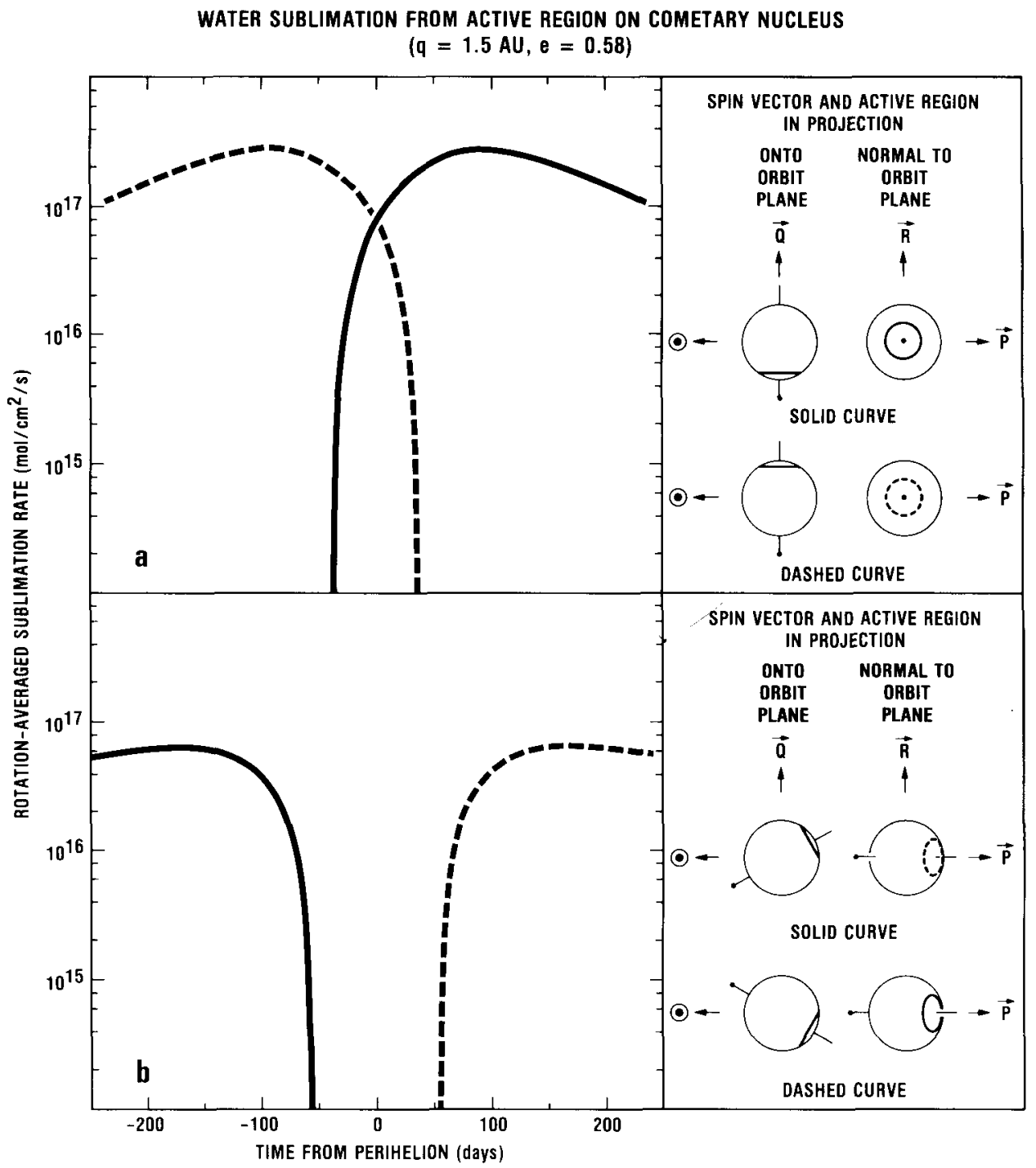

Figure 4. Calculated rotation-averaged sublimation curves for the nuclei of comets with a single active region on the surface. The parameters $\Phi, I$, and $\phi$ for the solid/dashed curves are: $0^{\circ}, 90^{\circ}$, $+60^{\circ} /-60^{\circ}$ for panel $a$ and $60^{\circ} / 120^{\circ}, 90^{\circ},-60^{\circ}$ for panel $b$. See the caption to Fig. 2 for further information. 
Application of the concept of a collimated flow of ejecta from discrete sources on the rotating nucleus to images of comet Encke's sunward fanlike coma (Fig. 1) resulted in the determination of the rotation-pole position, which appears to have changed little in the past several decades and is given by R.A.(1950) $=205^{\circ}$ and $\operatorname{Dec}(1950)=+2^{\circ}$ (equivalent to the argument $\Phi=230^{\circ}$ and obliquity $I=70^{\circ}$ at the epoch of 1980), with an estimated uncertainty of $5^{\circ}-10^{\circ}$ (Sekanina 1988b). Two active regions were identified on the nucleus: one, at an angular distance of $35^{\circ}$ from the northern rotation pole (latitude $+55^{\circ}$ ), which is found to dominate the comet's activity on the inbound leg of the orbit until several days before perihelion and again beginning some 2 months postperihelion; and the other, at a distance of $15^{\circ}$ from the southern pole (latitude $-75^{\circ}$ ), which is found to dominate in the intervening time near and after perihelion.

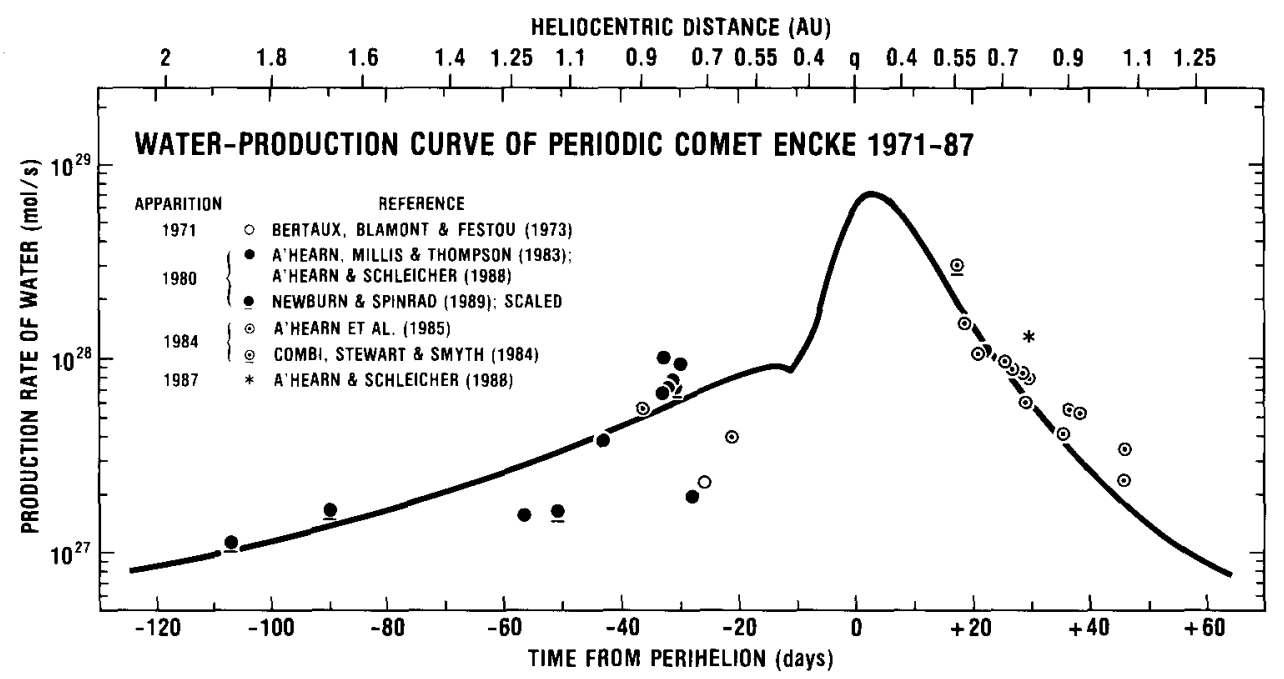

Figure 5. Production curve of water for Encke's comet at the apparitions of 1971-87. The various symbols represent reduced observations. The solid line is a rotation-averaged production curve of water calculated from the two-source model.

The production curve of water, calculated for Encke's comet from this model, is compared in Fig. 5 with available information, which consists primarily of the data summarized by A'Hearn, Millis, and Thompson (1983), by A'Hearn et al. (1985) (who, besides their own data, also included those by Bertaux, Blamont, and Festou 1973, by Bockelée-Morvan et al. 1981, and by Weaver et al. 1981), and by A'Hearn and Schleicher (1988). These sets are supplemented with the data by Newburn and Spinrad (1989; normalized to A'Hearn et al.'s production-rate scale) and by Combi, Stewart, and Smyth (1984). The observed emissions employed to derive the water-production rates are those of $\mathrm{OH}$ (in the $\mathrm{UV}$ and the microwave regions), the Lyman-alpha line of hydrogen, and the red line of [O I]. It should be noted that all the data on the water-production rate, although based on observations, are model-dependent (see the quoted references for the descriptions of the reduction methods). It should also be noted that the modelled, rotation-averaged water-production curve (the heavy curve in Fig. 5) is not a fit to the data points, except for vertical shifts that determine 
the surface areas of the active regions (assumed not to vary with time). The outgassing area of the north hemisphere's source was found to be $0.4 \mathrm{~km}^{2}$, and that of the south hemisphere's source, $0.6 \mathrm{~km}^{2}$.

The agreement between the model and the observations is generally quite good, but major, sudden jumps in the observed production are apparent along much of the inbound leg of the orbit and the drop near 30 days before perihelion, commented on by A'Hearn, Millis, and Thompson (1983), is almost certainly not the only one. These fluctuations, especially the ones during the several weeks immediately preceding the perihelion passage, can possibly be explained by the fact that the production-dominating north hemisphere's source is then in a transition from the polar-day regime (calculated to terminate 20 days before perihelion) to the polar-night regime (found to begin 2 days before perihelion). Almost simultaneously, the south hemisphere's region transits from a polar-night regime (which is calculated to end 12 days before perihelion) to a polar-day regime (to begin 5 days before perihelion). The argument is that even for some time before the termination of the polar-day regime, the diurnal variations in the insolation rate at the north hemisphere's active region become very large (calculated to reach, for example, a factor of 4 at 30 days before perihelion) because of the large difference between the Sun's elevations above the local horizon at noon and at midnight. This situation can be expected to result in major diurnal production variations, which could become erratic as the source struggles to adjust to the rapidly changing thermal conditions brought about by the approaching sunset/sunrise and polar-night regimes.

A'Hearn and Schleicher (1988) reported grossly disparate magnitudes of the Greenstein (1958) effect from analysis of high-dispersion spectra of Encke's comet taken with the International Ultraviolet Explorer about 33 days preperihelion in 1980 and 29 days postperihelion in 1987. From the intensity distribution in the $0-0$ band of the $\mathrm{OH}$ population in the coma, they found that the best match is achieved by models that imply a residual sunward radial component of $1.6_{-0.7}^{+0.3} \mathrm{~km} / \mathrm{s}$ in 1980 but only $0.3_{-0.6}^{+0.9} \mathrm{~km} / \mathrm{s}$ in 1987 , after the relevant component of the comet's orbital motion has been subtracted. These results obviously refer to the systematic velocity component of the parent molecules at the time of their dissociation, not at sublimation. The fairly large errors involved do not allow one to argue convincingly about perihelion asymmetry in the outgassing momentum, but A'Hearn and Schleicher's conclusion on the preperihelion fan pointing roughly toward the Sun to avoid unrealistically high expansion velocities is appropriate. The asymmetry, if it can be traced to the sublimation velocity (rather than to processes that accelerate water molecules between sublimation and dissociation) and if confirmed by future observations, would provide supporting evidence for the two-source model described.

The light curves of Encke's comet, based on more than 350 total magnitude estimates made by 37 selected visual observers between 1918 and 1987, are displayed in Fig. 6. The career observations by M. Beyer, J.E. Bortle, and A.F. Jones were essential for setting the magnitude-scale standards (based on the international photovisual system of magnitudes) to account for systematic personal and instrumental effects among all the observers. In the past, the comet's light curve was known for its perihelion asymmetry, in the sense that at the same heliocentric distance the object was intrinsically brighter inbound than outbound. This asymmetry is documented by brightness observations from earlier apparitions and especially by the comet's qualitative descriptions from the 19th century. Kamél (1989) has now found from his analysis of Encke's light curve that the object has recently been about equally bright before and after perihelion. Figure 6 shows that in the 1980s, the comet's postperihelion fading was indeed much less steep than it had been in the 1950s, the 1960s, 
HELIOCENTRIC DISTANCE (AU)

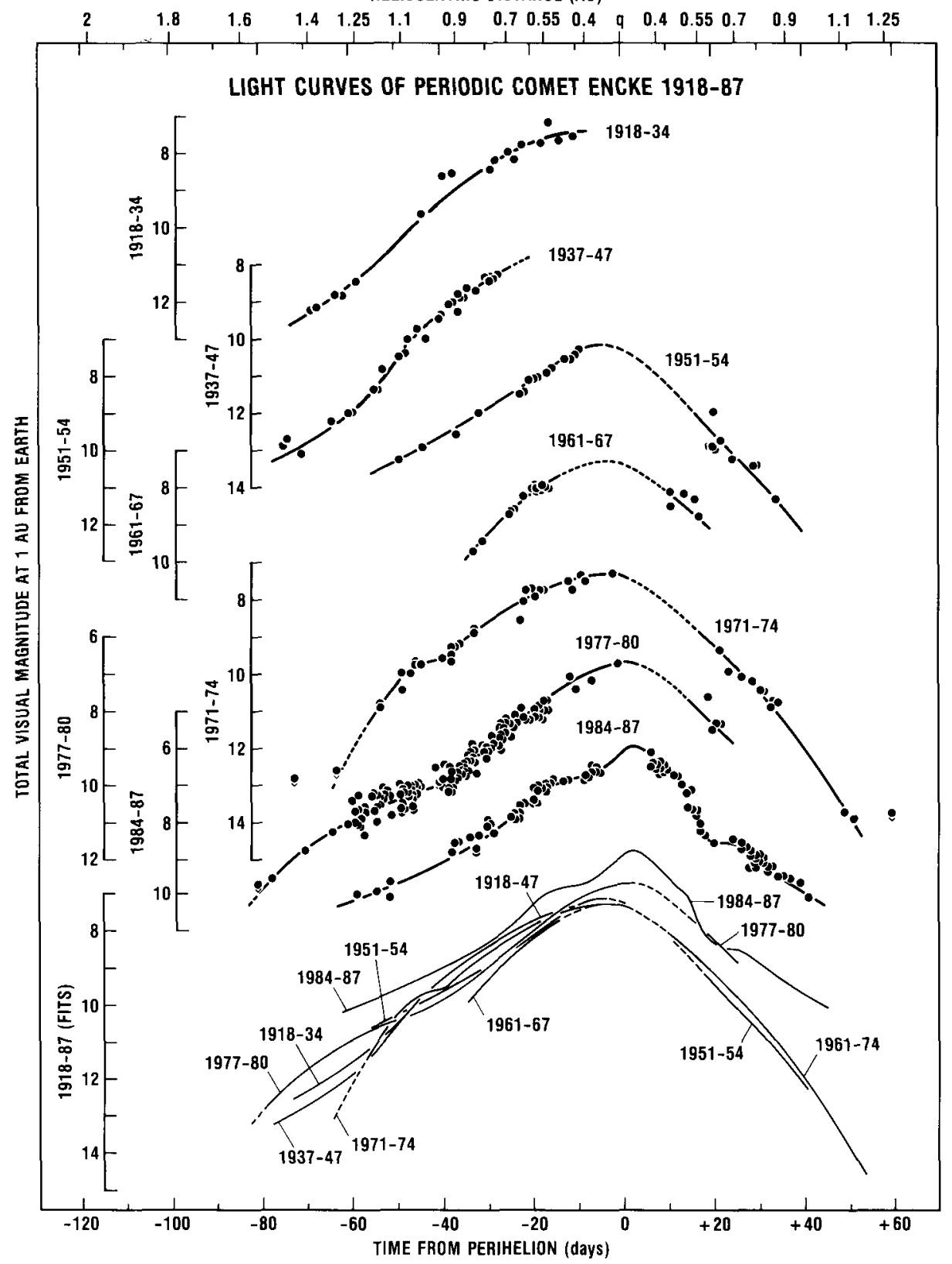

Figure 6. Visual light curves of Encke's comet at the apparitions 1918-87. The observations are normalized to $1 \mathrm{AU}$ from Earth and to a standard magnitude scale. Inverted carets indicate the data points are upper limits to the brightness (nondetections). The bottom panel compares the seven fitted light curves to show the long-term variations. 
and the early 1970s, and that the light curve's peak was shifting from before perihelion in 1951-54 to after perihelion in 1984-87. Figure 6 also contrasts the relative stability of the curve's inbound branch in the decades before 1950 with its changing shape more recently. For example, the arc of the curve that had been the steepest before 1950-at approximately 50 days before perihelion--became a saddle by 1980. Corroborating Kamél's (1989) results, Fig. 6 further indicates that Encke's comet has recently been growing brighter, a discrepancy with the previous apparitions that is particularly striking near and after perihelion. For the period 1951-87, spanning 11 revolutions about the Sun, this effect is illustrated in Table 2. Listing the parameters fitted to the standard empirical brightness law $r^{-n}$ ( $r$ is heliocentric distance), the table shows that Encke's intrinsic postperihelion brightness increased by at least 2 magnitudes between the early 1950s and the late 1980s, as seen from the magnitude $H_{0}$ (normalized to $1 \mathrm{AU}$ from the Sun and Earth). The light curves exhibit a parallel decrease in the slope $n$, reflecting the slower postperihelion fading in the $1980 \mathrm{~s}$. Even though Encke's water-production curve in Fig. 5 is not matched by the comet's light curves from the 1980 s, the discrepancy is substantially reduced compared with that displayed by the light curves from the earlier apparitions.

Table 2. Postperihelion light curves of comet Encke 1951-87.

\begin{tabular}{ccc}
\hline Apparition & $\begin{array}{c}\text { Normalized } \\
\text { magnitude } H_{0}\end{array}$ & $\begin{array}{c}\text { Heliocentric } \\
\text { slope } n\end{array}$ \\
\hline 1951 & $11.80 \pm 0.41$ & $4.58 \pm 1.02$ \\
$1954^{a}$ & $12.27 \pm 0.18$ & $(4.6)$ \\
& $11.93 \pm 0.13$ & $(3.9)$ \\
$1961^{a}$ & $11.86 \pm 0.45$ & $(4.6)$ \\
& $11.32 \pm 0.38$ & $(3.9)$ \\
$1974^{b}$ & $11.40 \pm 0.12$ & $3.93 \pm 0.40$ \\
$1977^{a}$ & $10.27 \pm 0.32$ & $(3.9)$ \\
& $9.75 \pm 0.33$ & $(2.9)$ \\
1984 & $9.81 \pm 0.08$ & $2.86 \pm 0.19$ \\
1987 & $9.72 \pm 0.05$ & $3.32 \pm 0.07$ \\
\hline \multicolumn{3}{c}{} \\
${ }^{a}$ Because of a short interval of heliocentric distance covered, $n$ could \\
not be determined from observations and is assumed. \\
${ }^{b}$ Observations satisfy the empirical law only at $r<0.9$ AU. Actual \\
$H_{0}>11.4$.
\end{tabular}

Figure 7 provides more information on perihelion asymmetry of Encke's activity in recent years, comparing the 1984-87 visual light curve with the 1984 production curves for $\mathrm{C}_{2}$ and CN, with the 1984 flux in the blue continuum (all from A'Hearn et al. 1985), and with the 1984 thermal infrared flux near $10 \mu \mathrm{m}$ that was reduced on two different assumptions by Campins (1988). Again, as with the water-production rates in Fig. 5 , the data on $\mathrm{C}_{2}, \mathrm{CN}_{\text {, }}$ etc. plotted in Fig. 7 are model-dependent quantities based on the original observations. The magnitude of the asymmetry as a function of time from perihelion is apparent from the degree of correspondence between the postperihelion data points and the mirror image of 
HELIOCENTRIC DISTANCE (AU)

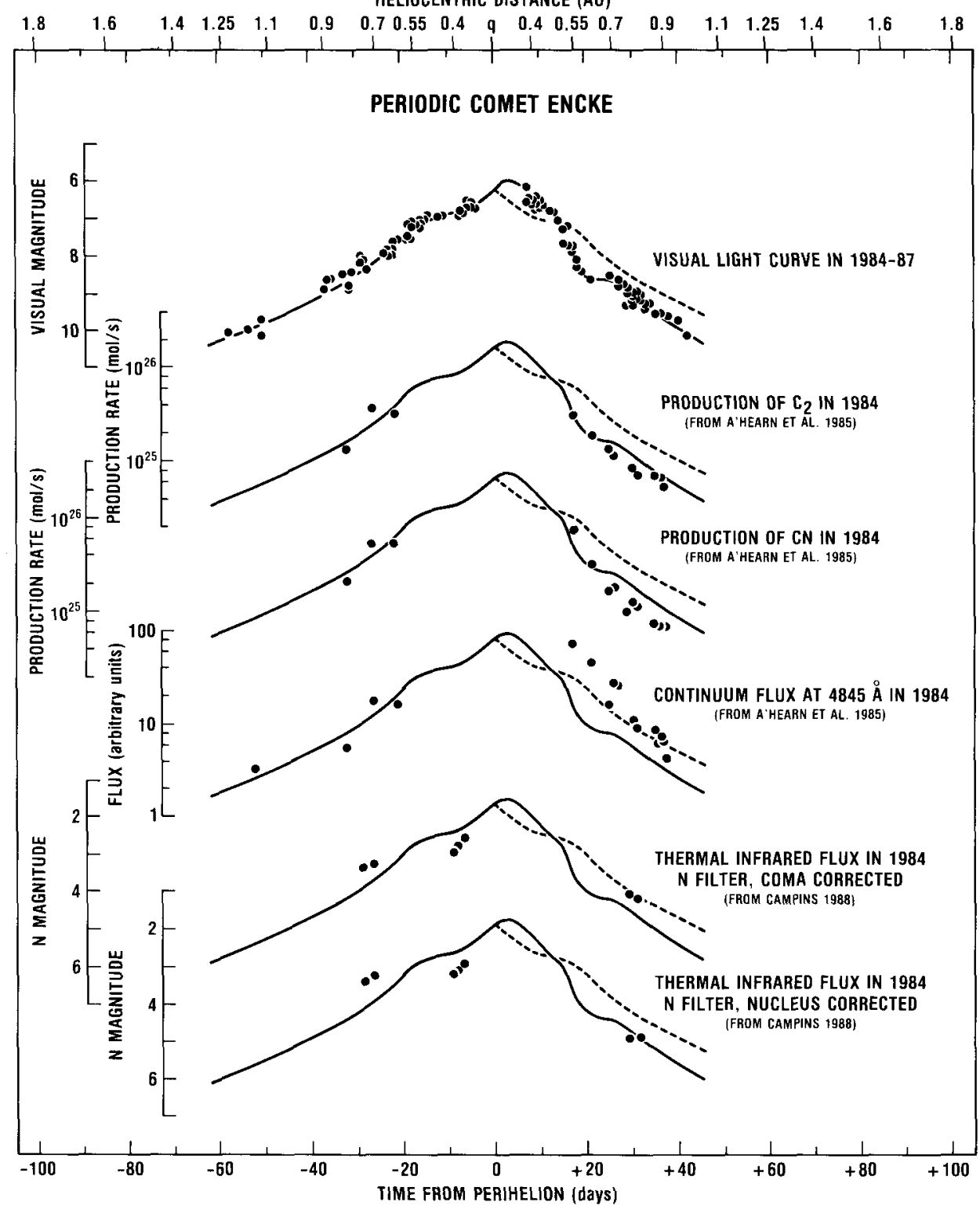

Figure 7. Comparison of the 1984-87 visual light curve of Encke's comet (cf. Fig. 6) with its production curves of $\mathrm{C}_{2}$ and $\mathrm{CN}$, continuum flux at $4845 \AA$, and thermal infrared flux at $10.6 \mu \mathrm{m}$ ( $N$ filter) in 1984. The flux data are corrected for the effects of aperture and geocentric distance. The solid curves are the fit to the visual light curve and the dashed curves show what its postperihelion shape would be if it were symmetric with respect to perihelion. The preperihelion branch of the fitted light curve is forced through the preperihelion data points of each of the production/flux curves to illustrate the degree of correlation. 
the preperihelion light curve, represented by the broken line. The light curve itself shows an excess brightness outbound within $\sim 13$ days of the perihelion passage, while the opposite is true at larger heliocentric distances. Beyond $\sim 25$ days from perihelion, the inbound and outbound branches run approximately parallel to each other, separated by some 0.6 magnitude on the average, out to at least 40 days from perihelion.

The degree of correlation between the visual light curve and the other characteristics of Encke's activity is illustrated in Fig. 7 by forcing the light curve through the preperihelion data points from 1984. A very good to fair match is found with the production curves of $\mathrm{C}_{2}$ and CN (confirming A'Hearn et al.'s 1985 conclusion), but the light curve is inconsistent with the other three curves. An excess continuum flux occurs throughout the outbound branch of the comet's orbit covered by the observations. The phase angle in 1984 varied from $110^{\circ}$ at 17 days after perihelion to $70^{\circ}$ three weeks later, so that the excess flux cannot readily be attributed to forward-scattering effects. Interestingly, the water-production curve in Fig. 5 is matched by the continuum-flux curve better than by any other curve in Fig. 7 . The preperihelion thermal infrared flux increases with decreasing heliocentric distance much too slowly compared with the light curve, but its interpretation is model-dependent and the temporal coverage very fragmentary (Campins 1988). With the exception of the correlation between the visual light and $\mathrm{C}_{2}$ (and, perhaps, $\mathrm{CN}$ ), more definite conclusions will have to await observations at Encke's future apparitions.

\subsection{COMETARY OUTBURSTS}

The term outburst is one of the most common and least well defined expressions used in cometary terminology. It is often applied quite indiscriminately and can indicate any rapid surge in brightness, a sudden increase in the production of a volatile species and/or dust, or even the appearance of a short-term phenomenon in a comet's head. Because of the usually inadequate temporal coverage by observations, the time scales of outbursts have rarely been determined with a meaningful accuracy and the variations in an object's physical behavior (including its appearance) during outbursts are known rather poorly. As an example, Table 3 lists descriptions of four events observed in Halley's comet in the period of 23-29 March 1986. Each of them either was, or could be, termed an outburst, even though their physical characteristics and time scales vary considerably.

Table 3. Outburst of comet Halley in late March 1986.

\begin{tabular}{|c|c|c|}
\hline Description of event & Dates (UT) & Reference(s) \\
\hline $\begin{array}{l}\mathrm{H}_{2} \mathrm{O} \text { infrared line flux increase by } 40 \text { per- } \\
\text { cent in } 1.8 \mathrm{hr}\end{array}$ & March 24.7 & $\begin{array}{l}\text { Weaver et al. (1986); Weaver, } \\
\text { Mumma, and Larson (1987) }\end{array}$ \\
\hline $\begin{array}{l}\text { Outburst associated with visual appear- } \\
\text { ance of sharp antisunward jet; duration } \\
\geq 10.7 \mathrm{hr}\end{array}$ & March 24-25 & Rettig et al. (1987) \\
\hline $\begin{array}{l}\text { Large but gradual flux increase by a factor } \\
\text { of at least } 2.5 \text { for } O H \text { and } 6 \text { for } C S \text { in } \\
1.2 \text { days }\end{array}$ & March 23-25 & McFadden et al. (1987) \\
\hline $\begin{array}{l}\text { OH burst at radio wavelengths; a rise for } \\
2 \text { days, a decrease for } 4 \text { days }\end{array}$ & March 24-29 & Silva \& Mirabel (1988) \\
\hline
\end{tabular}


Outbursts are a relatively frequent phenomenon amongst comets. In fact, Richter (1948) concluded from analysis of brightness flare-ups that a high proportion of unconfirmed comet discoveries may relate to very faint objects accidentally caught in outburst and promptly lost as their brightness subsides to normal level. Statistical investigations of outburst occurrences (e.g., Pittich 1972) show that most events are observed at heliocentric distances of $<2$ AU. However, since physical observations of comets at larger distances are significantly less complete than near the Sun, strong selection effects involved make statistical samples difficult to interpret.

Curiously, the object whose name is almost synonymous with outbursts-periodic comet Schwassmann-Wachmann 1-never gets anywhere near 2 AU of the Sun. Travelling in a nearly circular orbit just beyond Jupiter, the comet is known to flare up frequently by a factor of 100 or more with respect to its quiescent-phase brightness. As described by Richter (1941, 1954), Roemer (1958, 1962), Beyer (1962), Shao and Schwartz (1981), and others, the object appears absolutely stellar in the initial hours of an outburst. When observed photographically, the starlike condensation gradually expands into a disk or halo, which becomes diffuse with time and may display structure, sometimes of corkscrew shape. An essentially stellar nucleus is seen to be embedded in the expanding and fading halo. Eventually the structure disappears completely, leaving behind only a very faint, nearly stellar object, presumably the true nucleus in a state of low activity. The total brightness increases dramatically during the first hours after the onset of the outburst, reaching rapidly the peak and then subsiding at a slower rate. The apparent expansion velocity, as measured independently by Bobrovnikoff (1954), Richter, Roemer, Beyer, etc., is, in most cases, in a range of 100-200 m/s. From color variations with wavelength, Cochran, Cochran, and Barker (1982) determined the size distribution of dust particles, finding a typical grain size in the range of $0.1-1 \mu \mathrm{m}$. Although their result is model-dependent, it is consistent with the observed expansion velocities at $\sim 6 \mathrm{AU}$ from the Sun. The time scales of two events, one observed photographically by Roemer (1958) in August-September 1957 and the other visually by Beyer (1962) in October 1959-January 1960, differ dramatically. According to Roemer, the comet regains its quiescent appearance within 3-4 weeks, unless the outburst is followed by continuing activity. This appears to be what happened in late 1959, when Beyer reported the halo to be expanding steadily for the whole month, attaining about $10^{6} \mathrm{~km}$ in diameter at the maximum. The total brightness, having reached its peak in a few days after the onset of the outburst, did not begin to subside appreciably until a month or so later. The whole event extended over a period of more than three months and the apparent short-term variations in brightness when at its maximum did indeed suggest the occurrence of multiple outbursts. This is nothing unusual, since the comet was repeatedly reported to display essentially continuous low activity over very extended periods of time (Roemer 1958; Roemer, Thomas, and Lloyd 1966; Shao 1981; Jewitt 1990). In earlier times, occasional spectra obtained when the comet was in outburst always showed a pure continuum, with no emissions whatsoever (Mayall 1941; Jeffers 1946; Walker 1959). More recently, however, a $\mathrm{CO}^{+}$emission was detected both during an outburst and in the quiescent phase, along with the continuum (Cochran, Barker, and Cochran 1980; Larson 1980; Cochran, Cochran, and Barker 1982). The corkscrew-shaped nebulosity referred to above is illustrated on the comet's image in Fig. 1. The halo's maximum diameter at the time of exposure was almost $150,000 \mathrm{~km}$, and it is estimated that the outburst had begun about one week before this image was obtained. The reader is referred to Roemer (1958) for more details on this comet's appearance and behavior. 
A number of interpretations have been offered in the literature, the principal issue being that of the energy sources needed for the outbursts. Richter (1954) reported a correlation between the outbursts of comet Schwassmann-Wachmann 1 and terrestrial magnetic storms, implying a role of solar corpuscular streams. However, the concept was criticized (e.g., Weigert 1959) and the relationship between the comet's outbursts and solar activity was not confirmed by subsequent investigations (e.g., Cochran, Cochran, and Barker 1982). Whitney (1955) proposed the existence, in a comet nucleus, of pockets of highly volatile species, in which pressure would build up and lead to explosions-a notion to which Huebner (1975) also subscribed to explain jet activity in general. Donn and Urey (1956) suggested exothermic chemical reactions that involve radicals, Feldman et al. (1986) suspected a role of $\mathrm{CO}_{2}$, while Harwit (1968) speculated that the trigger could be random collisions with interplanetary boulders - an ad hoc hypothesis. On the other hand, Patashnick, Rupprecht, and Schuerman (1974) came up with the idea of phase transition of water ice, from amorphous below about $140^{\circ} \mathrm{K}$ to crystalline (cubic) at higher temperatures. This process is exothermic, giving off about $24 \mathrm{cal} / \mathrm{g}$, an amount of energy that is consistent with the observed velocities of expanding halos in the outbursts of comet SchwassmannWachmann 1. Smoluchowski (1981a) argued in favor of the same mechanism, considering the roles of porosity and nucleus rotation. Prialnik and Bar-Nun (1987) recently confirmed that the phase transition constitutes a major internal source of energy, showing that in pure-ice nuclei the process occurs in distinct steps and that some of the gas trapped in the amorphous ice accumulates in pockets that may eventually explode. Further extending Patashnick et al.'s ideas, Jewitt (1990) suggested that sublimation of the crust of crystalline ice accounts for the persistent low-activity coma of comet Schwassmann-Wachmann 1, while an occasional local exposure of the subsurface amorphous ice triggers an outburst, which is subsequently quenched by the phase change. This model requires that much of the nuclear surface be covered with water ice (instead of an inert dust mantle) and it predicts that low activity is present at all times.

It is sometimes argued that the propensity for outbursts is an inherent property of comet Schwassmann-Wachmann 1 , since they have been completely absent in periodic comet Oterma, which, until recently, had travelled about the Sun in a nearly circular orbit of similar, but slightly smaller dimensions. While the phase change of water ice, if indeed relevant, may make the outbursts critically temperature-dependent (and therefore solardistance-dependent), it also is plausible that outbursts in different comets have different causes. For comet Schwassmann-Wachmann 1, Whipple (1980) found that only one large area of the nucleus is usually active during one opposition and argued that the activation process may involve sudden exposure of amorphous ice caused by erratic phenomena, such as collapse of pedestals or small meteoroid impacts. He proposed that because of the large size of this comet's nucleus, more sizable fragments of the broken crust reach velocities too low to escape but high enough to break more crust, thus providing a trigger and a chain-reaction process. To terminate an outburst, Whipple resorted to the sort of heatstorage mechanism that was suggested by Whitney. On the other hand, to explain two huge outbursts (brightness jumps by a factor of more than 1000) of periodic comet Holmes in 1892-93, Whipple (1984) considered a grazing encounter of a small companion with the principal nucleus, followed by their collision on the next passage, with each event activating new areas on the surface of the main mass.

Because of the angular momentum differences between the orbits of a comet and its particulate ejecta, the comet's history of dust production is, for a limited period of time, 
preserved in the light distribution of its dust tail. In particular, sudden outbursts can readily be recognized as narrow streamers extending from the nucleus to the tail's extremity and the outburst times determined from the orientation of these tail structures. For details, the reader is referred to another review (Sekanina 1980). This method was successfully applied to several comets, including Halley (Lamy 1986; Sekanina 1986b; Beisser and Boehnhardt 1987) and West 1976 VI, for which the times of outbursts were shown to match closely those of nucleus breakups (Sekanina and Farrell 1978). Correlations between outbursts and splitting were also documented for other comets; cf. Sekanina (1982) for a review.

It thus seems fairly probable that there is no single cause for cometary outbursts. Rather, different mechanisms appear to be responsible for outbursts in different comets, and more than one process may bring about outbursts even in the same comet. Thanks especially to the enormous amount of information accumulated on Halley's comet, there is now no doubt that some of the alleged outbursts are merely products of diurnal and seasonal variations experienced by the discrete active regions on the morphologically heterogeneous nuclei of comets (Sec. 4.2).

\subsection{DISAPPEARANCE OF COMETS}

A relatively small group of objects, which have been called dissipating comets (Sekanina 1984a), are observed to undergo major physical changes and dramatic variations in their activity on the time scales of several days to a few weeks. When discovered, these comets often display a prominent, starlike central condensation, probably indicating that an outburst is in progress. The fading sets in very suddenly and the condensation disappears usually in a matter of days. At the same time, the coma expands gradually and becomes progressively elongated. Its surface brightness decreases (sometimes with erratic variations superimposed) until the comet's head completely vanishes. The dust tail can become the brightest part of the comet and survive the head. There is evidence that the dramatic changes in the appearance of some of these objects are triggered by a prediscovery eruptive event that facilitates (and may, in some cases, become essential for) the comet's detection-a scenario that is reminiscent of Richter's (1948) suggestion regarding the unconfirmed comet discoveries (Sec. 4.4).

The most puzzling property of the dissipating comets is that the process of disappearance proceeds at such an alarmingly fast pace and typically without any warning. The terminal changes experienced by these objects bear a resemblance to the physical behavior of the secondary nuclei of the split comets (Sekanina 1982). The most important similarity is in the fact that none of the dissipating comets and none of the secondary nuclei have ever been observed again following their disappearance. By contrast, there is no evidence whatsoever that outbursts - regardless of their magnitude and frequency distribution-are, by themselves, a sign of imminent disintegration.

It has been stated in Sec. 4.2 that a phenomenon often described by observers as the disappearance of a comet can formally be simulated-noncatastrophically-as an effect of seasonal variations. While this is correct, the global physical characteristics displayed by the dissipating comets and, in particular, the fact that they are never observed again indicate that a true physical disintegration may be involved. Unfortunately, the investigation of these intriguing objects is frustrated by their very nature: the sudden disappearance, often near the perihelion point, abruptly terminates all observations, physical as well as positional. As a result, information on these comets' physical evolution and orbital motion is inevitably incomplete. 


\section{Evolution of Cometary Nuclei With Discrete Active Regions}

Theoretical and observational evidence reviewed in Sec. 4 illustrates a tremendous diversity of physical behavior that morphologically heterogeneous, nonuniformly outgassing nuclei of comets can generate. From what has been said, it already is obvious that the existence of discrete centers of activity has important implications for the physical appearance of comets. This section summarizes information that offers further insights into the structure of the nuclear surface and the processes involved, and integrates this information with plausible conjectures on the long-term evolution of cometary nuclei.

\subsection{NUCLEUS SHAPE}

Traditionally, cometary nuclei were modelled as spherical objects. The assumption of an oblate nucleus was introduced by Whipple and Sekanina (1979) for Encke's comet in an effort to interpret the nongravitational perturbations of its orbital motion as a product of torques exerted by anisotropic outgassing (Sec. 5.5). Reviewing the subject of the split comets, Sekanina (1982) concluded from a disparity between the large differential nongravitational effects and the small brightness differences among the individual fragments that especially the minor companions should be considerably nonspherical, possibly pancake-shaped, and that the parent nuclei have a tendency to "peel off" rather than to break up. Recent detections of large periodic variations in the visual/infrared light curves of several lowactivity comets have been interpreted consistently as being due to a changing projected cross-sectional area of the rotating nucleus viewed from a point not too distant from its equatorial plane (cf. A'Hearn 1988; A'Hearn et al. 1989; Jewitt and Luu 1989). These observations show decidedly that comet nuclei are neither spheres nor oblate spheroids rotating about the axis of maximum moment of inertia. The figures of two comet nuclei were successfully approximated by triaxial ellipsoids (Goldstein, Jurgens, and Sekanina 1984; Sekanina 1988a,d). Direct imaging of Halley's nucleus showed it to be, in fact, quite irregular (cf. Szegö's paper in these proceedings), although it can be very crudely approximated by a prolate spheroid, in the sense that the two short body axes are about equal (but not in the sense that the longest axis should coincide with the principal rotation axis).

Nucleus shape plays a role in considerations of the formation and evolution of comets. Low-velocity random accretion of comets from grain aggregates, which can be described by the fractal model, was recently proposed by Donn and Hughes (1986) to explain some of the properties of cometary nuclei. An advantage of this formalism is the availability in the literature of random-walk schemes for growing fractal structures by numerical experiments, with implications for shape, porosity, and other physical signatures of the aggregates. Donn, Daniels, and Hughes (1985) and Donn and Hughes (1986) argued that the results of the computer simulations of dust growth presented by Daniels and Hughes (1981) could be scaled up to dimensions of cometary nuclei after allowing for some compression. One of the conclusions of the 1981 study was that random accretion leads to generally spherical aggregates, although occasional significant deviations from sphericity were detected during growth. However, nonsphericity in Daniels and Hughes' (1981) investigation means random irregularities in an aggregate's shape, not a major elongation in any particular direction. Jewitt and Meech (1988b) found, from their numerical experiments restricted to a constant size of impinging grains, that aggregates of $\sim 1000$ of these "cometesimals" often exhibit approximately triaxial or pancake-shaped outlines. Obviously, nucleus shape remains a major issue to be addressed in greater detail in the future. 


\subsection{OUTGASSING FRACTION OF THE NUCLEUS SURFACE}

As already pointed out at the beginning of Sec. 4, the outgassing surface area of a cometary nucleus is determined as a ratio of the measured production rate and the calculated sublimation rate from a unit surface area. In practice, the outgassing areas are calculated from information available on water production. The results-still very incomplete and uncertain - are usually presented as a fraction of the nucleus surface, whose estimate is unfortunately an additional source of error. Furthermore, because of uneven and changing exposure of the nucleus to the Sun, one should distinguish between the fraction of the surface that actually is active at a given time and an "integrated" outgassing area, which is the sum of the areas of the individual regions, each of which becomes active at one time or another during a reasonably long period, such as one revolution about the Sun. Most of the entries in Table 4 describe the state of outgassing on short time scales.

Strictly, the sublimation rates used to calculate the extent of the active areas should be rotation-averaged, requiring the knowledge of the spin-axis orientation and the location of each discrete source on the nucleus surface, and should further be corrected for an enhancement effect that arises from thermal energy trapped in concave topographic features, such as craters or other depressions. This focussing was demonstrated by Colwell and Jakosky's (1987) modelling of sublimation from an infinite cylindrical trench as a function of its diameter and depth with respect to the surrounding surface. Their model is based on a standard steady-state sublimation theory with no heat conduction into the nucleus, but it includes such physical processes as shadowing, radiative heating from the opposite walls of the trench, and the condensation energy of the gas striking the walls. The major conclusion of Colwell and Jakosky's study is that the sublimation rate from such a depression is greater than that from an equivalent flat surface and that the maximum effect occurs at the trench's floor, except in deep depressions (diameter-to-depth ratio of 2:1 or less), whose shape is essentially maintained because less direct sunlight reaches the floor. Colwell and Jakosky calculated that the relative excess at the floor over the sublimation rate from a flat surface is about 30 percent for a diameter-to-depth ratio of 3.5 at $1 \mathrm{AU}$ from the Sun and that this excess increases with heliocentric distance and-for shallower depressions-also with the reciprocal diameter-to-depth ratio. Since the results in Table 4 are only crude estimates, one can neglect this effect with the caveat that the tabulated entries are, in fact, upper limits to the probable values of the outgassing surface area.

Rotation information is not available for some of the comets in Table 4, and their sublimation rates were approximated by the average values over the sunlit hemisphere. Even for the best entries in Table 4, the locations of the discrete outgassing areas are de facto unknown and were assumed to coincide with the locations of the observed dust sources. The data for comets Swift-Tuttle, Pons-Winnecke, Schwassmann-Wachmann 3, and Machholz are particularly uncertain, since the water-production rates for these objects were determined indirectly and not from observations of the dissociation products of water. With the exception of the first three entries on the list, outgassing is found to proceed from only a small fraction of the nucleus surface. For a larger number of comets, the extent of their outgassing areas was calculated on similarly simplified assumptions by Newburn and Spinrad (1989), who found major temporal variations and a range generally between 0.1 and $10 \mathrm{~km}^{2}$, with Halley's comet being the only exception, and by Rickman et al. (1987), who listed-besides Halley-only three short-period comets (among nearly 30 ) with their outgassing areas greater than $10 \mathrm{~km}^{2}$. 
Table 4. Outgassing fraction of nucleus surface.

\begin{tabular}{|c|c|c|}
\hline Comet $^{a}$ & $\begin{array}{c}\text { Fraction of } \\
\text { nucleus surface } \\
\text { active (percent) }\end{array}$ & Reference(s) \\
\hline P/Kopff & 30 & Rickman et al. (1987) \\
\hline P/Giacobini-Zinner & 24 & Rickman et al. (1987) \\
\hline Sugano-Saigusa-Fujikawa (1983 V & $\gg 20$ & Hanner et al. (1987a) \\
\hline $\mathrm{P} /$ Halley & $\sim 10$ & Keller et al. (1986) \\
\hline P/Crommelin & 9 & Rickman et al. (1987) \\
\hline $\mathrm{P} /$ Encke & $\begin{array}{c}5-10 \\
<10 \\
2.6 \\
\sim 1 \\
0.5-1.3\end{array}$ & $\begin{array}{l}\text { Delsemme \& Rud (1973); Delsemme (1982) } \\
\text { Fanale \& Salvail (1984) } \\
\text { Rickman et al. (1987) } \\
\text { Jewitt \& Meech (1987) } \\
\text { Sekanina (1988b) }\end{array}$ \\
\hline P/Schwassmann-Wachmann 3 & (6) & Sekanina (1989) \\
\hline P/Pons-Winnecke & $3-7$ & Sekanina (1989) \\
\hline P/Tempel 2 & $\begin{aligned} & 5 \\
& 4 \\
\sim & 0.5 \\
0.15-1.5 & \sim 1 \\
& \sim 1\end{aligned}$ & $\begin{array}{l}\text { Rickman et al. (1987) } \\
\text { Sekanina (1987c) } \\
\text { Sekanina (1988d) } \\
\text { Jewitt \& Luu (1989) } \\
\text { A'Hearn et al. (1989) }\end{array}$ \\
\hline IRAS-Araki-Alcock (1983 VII) & $\begin{array}{c}1 \\
0.2-1\end{array}$ & $\begin{array}{l}\text { Hanner et al. (1985) } \\
\text { Sekanina (1988a) }\end{array}$ \\
\hline P/Machholz & $\sim \underset{4-6^{b}}{\sim 1}$ & $\begin{array}{l}\text { Sekanina }(1990) \\
\text { Sekanina }(1990)\end{array}$ \\
\hline P/Swift-Tuttle & $\lesssim 1$ & Sekanina (1981b) \\
\hline P/Tempel 1 & $\leq 1$ & Luu \& Jewitt (1988) \\
\hline P/Arend-Rigaux & $\ll 1$ & Millis, A'Hearn \& Campins (1988) \\
\hline P/Grigg-Skjellerup & 0.8 & Rickman et al. (1987) \\
\hline $\mathrm{P} /$ Neujmin 1 & $0.1-0.3$ & Campins, A'Hearn \& McFadden (1987) \\
\hline
\end{tabular}

a The symbol P/ means that the comet has an orbital period of less than 200 years.

${ }^{b}$ Maximum outgassing area during an outburst.

Supported both by the results from the closeup imaging observations of Halley's comet and by very low albedos that were derived for the nuclei of several comets from the combined ground-based visual/infrared brightness observations (cf. A'Hearn 1988 for a review), a consensus appears to have emerged that the small extent of the outgassing areas is evidence for the presence of extensive, dark, inert mantles on cometary nuclei. The issues of the mantle's formation, propagation, thickness, and long-term evolution are still largely in the realm of speculation and are not addressed in any detail in this review. The reader is referred to a number of papers that describe the mantle formation as a product of the sublimation process (e.g., Brin and Mendis 1979; Brin 1980; Fanale and Salvail 1984; Ip and Rickman 1986; Rickman and Fernández 1986; Wallis and Wickramasinghe 1987) or as a product of the cosmic-ray irradiation processes during the stay of comets in the Oort cloud (e.g., Donn 1976; Johnson et al. 1987; Sekanina 1987a). 


\subsection{EROSION RATES OF THE ACTIVE REGIONS}

Free sublimation of water and other ices and ejection of the dust entrained in the gas flow gradually erode the surface of discrete active regions. One can expect that especially the regions that have been active for a long time should appear as major depressions with respect to the surrounding mantled areas. With certain assumptions, it is possible to estimate an active region's erosion rate per revolution about the Sun as a function of the comet's orbital dimensions, the nucleus spin vector, and the region's location on the nucleus surface. This erosion rate is a measure of the potential life span of the source and, indirectly, it yields information on the history of a comet's activity.

To investigate the erosion rate quantitatively, let us assume that gas and dust emission proceeds from $k$ active regions on the nucleus. If $Z_{i, j}(t)$ is the representative sublimation rate, at time $t$, of the $j$-th volatile species $(j=1, \ldots, m)$ from the $i$-th active region $(i=1, \ldots, k)$, the region's total erosion rate per revolution is

$$
E_{i}=\frac{1+\psi_{i}}{\rho_{i}} \sum_{j=1}^{m} \mu_{j} \int_{\left(P_{\text {orb }}\right)} Z_{i, j}(t) d t
$$

where $\mu_{j}$ is the mass of a molecule (or atom) of the $j$-th volatile species, $P_{\text {orb }}$ indicates that the expression is integrated over the entire orbit, and $\psi_{i}$ and $\rho_{i}$ are the dust-to-ice ratio by mass and the bulk density of the material in the active region before evacuation. The total mass lost by the comet per revolution is

$$
\Delta M=\sum_{i=1}^{k} A_{i} E_{i} \rho_{i}
$$

where $A_{i}$ is the effective outgassing area of the $i$-th active region.

Figure 8 is a plot of the total erosion effect during one revolution about the Sun. The individual active regions on the nuclei of several comets are identified by their cometographic latitudes. The erosion rate was derived on the assumptions that the outgassing proceeds whenever the Sun is above the local horizon and that the activity's variable rate is determined by the changing local energy budget. If these assumptions are not satisfied, the erosion rate as plotted may represent an upper limit. Only the rate of water production was actually calculated, the contributions by the other volatile species and by the dust being accounted for by application of approximate scaling factors. Information on the rotation and on the locations of the active regions on the nuclei of the comets plotted in Fig. 8 was taken from Sekanina (1987c, 1988a,b,c, 1989) for Tempel 2, IRAS-Araki-Alcock, Encke, PonsWinnecke, and Schwassmann-Wachmann 3, while the result for comet Halley is presented as a function of cometographic latitude, based on the rotation parameters by Wilhelm et al. (1986). The ratio of the total erosion rate to that of water was conservatively estimated at 2.1 for Halley, 1.8 for Tempel 2, Pons-Winnecke, and Schwassmann-Wachmann 3, and 1.2 and 1.1 for the north hemisphere's and the south hemisphere's regions (Sources I and II) of Encke's comet, respectively.

The erosion rate depends critically upon the local bulk density. Attempts have so far been made to estimate only an average bulk density of the whole nucleus and no information is available on its variations, horizontal or vertical. Even the average density determinations are quite uncertain and the quantity may vary significantly from comet to comet. 
Yet, several investigators independently arrived at densities in the range of $0.2-0.3 \mathrm{~g} / \mathrm{cm}^{3}$, employing very different lines of evidence. A density of about $0.3 \mathrm{~g} / \mathrm{cm}^{3}$ was advocated for aggregated frozen gases in cometary nuclei by Donn (1963), primarily because of the low accretion velocities involved. From evidence on the ablation of meteors in the Earth's atmosphere, Öpik (1958 and references therein) concluded that meteoroids of cometary origin are typically loose aggregates of dust particles, called by him "stoneflakes" or "dustballs". More recent studies of meteors (e.g., Ceplecha 1986) showed that there are at least two categories of cometary meteoroids-Types IIIa and IIIb-and analysis of atmospheric paths of very bright meteors (fireballs) indicated that their bulk densities are $0.6-0.9 \mathrm{~g} / \mathrm{cm}^{3}$ for Type IIIa and $0.2-0.34 \mathrm{~g} / \mathrm{cm}^{3}$ for Type IIIb. The Draconids-the stream associated with periodic comet Giacobini-Zinner-belong entirely to the low-density group. This is significant, because the Draconids contain the most pristine cometary ejecta that Earth encounters: they could not have been expelled from the parent comet long before 1900 and are therefore by far the least modified by processing in interplanetary space.

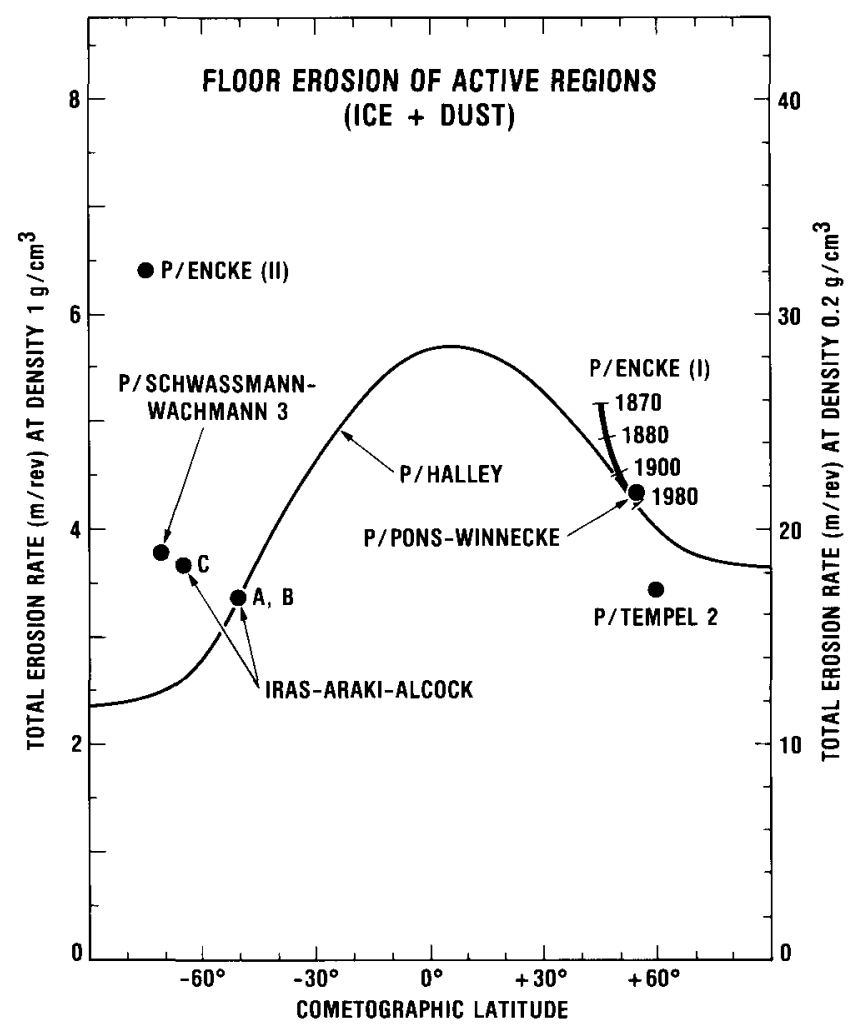

Figure 8. Calculated total erosion rate of discrete active regions on the nuclei of six comets. P/ refers to comets that have an orbital period of less than 200 years. For comet IRAS-Araki-Alcock, the three active regions are identified as A, B and C (Sekanina 1988a). For Encke's comet, the two active regions are marked $I$ and II (cf. Sec. 4.3). The heavy curve for the region I shows the effects of the source's inferred migration and the gradual variations in its outgassing rate due to nuclear precession between 1870 and 1980. The lighter curve for Halley's comet depicts the expected variations in the erosion rate with latitude, assuming an inertially fixed orientation of the spin axis determined by Wilhelm et al. (1986). The two scales illustrate the effect on the erosion rate of the material bulk density in the active regions before their evacuation.

The approach most commonly employed to appraise the bulk density of cometary nuclei utilizes the dynamical nucleus-mass determination based on modelling the nongravitational perturbations of the orbital motions of periodic comets (cf. Sec. 5.5). A density lower than 
$0.5-0.7 \mathrm{~g} / \mathrm{cm}^{3}$ was advocated by Wallis and Macpherson (1981) from comparison of their model for jet thrusting of comets with Whipple's (1977) results to reconcile the nongravitational forces with free sublimation of water. However, in the early 1980s, the volumes of cometary nuclei were still largely unknown and Wallis and Macpherson's arguments were not very compelling under the circumstances. The situation changed after the nucleus of Halley's comet was imaged with spacecraft cameras and when its mass became the only quantity in the expression for its bulk density to be derived. Taking account in his calculations of the perihelion asymmetry of gas production, Rickman (1986) concluded that the bulk density of Halley's nucleus is merely $0.1-0.2 \mathrm{~g} / \mathrm{cm}^{3}$ and that periodic comet Kopff apparently has a nucleus of an even lower density. In a follow-up investigation, Rickman et al. (1987) determined the bulk density for Encke's comet to be $0.09-0.15 \mathrm{~g} / \mathrm{cm}^{3}$ and the upper limits to the density of periodic comets Kopff, Tempel 2, Giacobini-Zinner, and GriggSkjellerup to be $0.10,0.54,0.19$, and $0.04 \mathrm{~g} / \mathrm{cm}^{3}$, respectively. Sagdeev, Elyasberg, and Moroz (1988), employing the same approach with somewhat different assumptions regarding the details of the outgassing mechanism, concluded that, depending upon the emphasis assigned to the various sources of information on the comet's water production, the bulk density of Halley's comet could be anywhere between 0.2 and $1.5 \mathrm{~g} / \mathrm{cm}^{3}$. However, the maximum model-which gives the upper limit to the density-is seen on Sagdeev et al.'s Fig. 2 to overestimate grossly the perihelion asymmetry of water production. Subsequently, Rickman (1989) reanalyzed the problem, fitting two curves through the data points and finding, respectively, the densities of $0.28 \pm 0.10$ and $0.65 \pm 0.19 \mathrm{~g} / \mathrm{cm}^{3}$.

Other investigators likewise favor a low density and high porosity for cometary nuclei. Describing them as fluffy, loosely packed structures, Greenberg (1986) argued that at least 60 percent of their volume is vacuum, that their mean density amounts to less than $0.5 \mathrm{~g} / \mathrm{cm}^{3}$, and that very little internal heating is involved. Sekanina and Yeomans (1985) concluded that the tidal splitting of periodic comet Brooks 2 near Jupiter in 1889 requires the comet's bulk density to be in the range of $0.25 \pm 0.08 \mathrm{~g} / \mathrm{cm}^{3}$. Further density estimates are provided by studies of the particulate material, especially in Halley's comet. Krasnopolsky et al. (1987) evaluated the density of dust particles in this object from comparison of the dust-impact measurements with the data obtained with a three-channel spectrometer on VEGA-2, finding a value of $\sim 0.3 \mathrm{~g} / \mathrm{cm}^{3}$ with an uncertainty of about a factor of 2 . Crifo (1988) arrived at a density of $0.3 \mathrm{~g} / \mathrm{cm}^{3}$ or less for Halley's dust grains from his fitting the observed thermal infrared emission features in the 10-20 $\mu \mathrm{m}$ region. Smirnov, Vaisberg, and Anisimov (1987) concluded from their analysis of the foil penetration of the dust sensors on VEGA-1 and VEGA-2 by Halley's impacting dust that particles less massive than $\sim 10^{-14} \mathrm{~g}$ had a density of $0.1-0.3 \mathrm{~g} / \mathrm{cm}^{3}$ in the outer coma and closer to $1 \mathrm{~g} / \mathrm{cm}^{3}$ in the inner coma and that particles more massive than $\sim 10^{-12} \mathrm{~g}$ were mostly of very low density or complicated structure. On the other hand, Maas, Krueger, and Kissel (1989), deriving particle densities from more than 100 mass spectra collected by the PUMA experiment on VEGA-1, obtained a mean value of $2.5 \mathrm{~g} / \mathrm{cm}^{3}$ for the silicate-dominated grains and $1 \mathrm{~g} / \mathrm{cm}^{3}$ for the CHON (light-element enriched) grains; for both particle classes, the mass range is generally $10^{-15}-10^{-12} \mathrm{~g}$. It should be pointed out, however, that all the density determinations have been indirect (involving various assumptions and/or extrapolations) and that, in addition, the relevance of the particle-density determinations to the problem of the comet's bulk density depends strongly upon the characteristic scale and distribution of voids in the nuclear interior. In general, particle-density determinations offer only an upper limit to the global bulk density. 
A lower limit to the bulk density of cometary nuclei is sometimes derived from a simple condition that the centrifugal acceleration due to rotation not exceed the centripetal acceleration due to comet gravity, to prevent the development of internal tensile stresses in the nucleus. Jewitt and Luu (1989) applied this criterion to the nucleus of periodic comet Tempel 2 to find a "critical" density of $0.3 \mathrm{~g} / \mathrm{cm}^{3}$. Contrary to the authors' claim, however, this limit is virtually meaningless, because cometary nuclei do possess some internal strength and the maximum tension that develops in the equatorial plane of a rotating nucleus of low density is very small, on the order of $10^{2} \mathrm{dyn} / \mathrm{cm}^{2}$ in the case of comet Tempel 2. One should also keep in mind that in the direction of the rotation poles, the nucleus withstands - regardless of its spin rate-a compression stress due to gravitation pressure, whose magnitude is comparable with, or greater than, the maximum tensile stress in the equatorial plane.

If the arguments in favor of a low bulk density-about $0.2 \mathrm{~g} / \mathrm{cm}^{3}$ or so-are accepted, the total erosion rate from Fig. 8 is typically $20 \mathrm{~m}$ per revolution. Within the uncertainties involved, this result is on the conservative side of the independent estimates of $30-40 \mathrm{~m}$ per revolution for Halley's comet (Rickman 1989; Reitsema et al. 1989). The focussing effect in concave surface depressions, which was discussed by Colwell and Jakosky (1987) but was not accounted for here (Sec. 5.2), also suggests that the erosion rates in Fig. 8 are conservative. For Encke's comet, whose nucleus radius almost certainly does not exceed $3 \mathrm{~km}$ (Campins 1988), the calculated erosion effect implies that, if continuing at this high rate, the depth of evacuated depressions should approach the dimensions of the nucleus in only a hundred or so revolutions about the Sun. This contrasts with the age of a meteor stream associated with this comet, which is estimated at $4700 \mathrm{yr}$, or about 1400 revolutions about the Sun (Whipple and Hamid 1952), and which suggests that the comet has been in its short-period orbit longer than this. It is apparent that active regions on the nuclei of the comets of Jupiter's family, especially those with small perihelion distances, must evolve relatively rapidly and have fairly short life spans, unless the adopted nucleus bulk density is grossly underestimated.

Numerous examples of variations in cometary activity on the relevant time scales of tens of years and longer are available in the literature. For example, periodic comet ArendRigaux, which had a tenuous coma when discovered in 1951 (and was therefore classified as a comet), was reported as an object of perfectly stellar appearance at three subsequent apparitions between 1958 and 1970 (and would have been classified as an asteroid, if it had been missed in 1951), but activity was detected again at the apparitions of 1977 and 1984 (cf. Millis, A'Hearn, and Campins 1988 for references to the apparitions between 1951 and 1984). Much attention was recently paid to the detection of a coma of the slow-moving object (2060) Chiron (Meech and Belton 1989), which orbits the Sun between Saturn and Uranus and which has, since its discovery, been classified as an asteroid. For still longer time scales, circumstantial evidence was presented by Kresák (1987) for dormant phases in the evolution of seven short-period comets from the existence of favorable observing conditions at some of their prediscovery returns to the Sun. It is apparent that over long periods of time and over a wide range of heliocentric distance, an active comet may turn into a dormant object and vice versa. Evidence of these transformations in the appearance of a relatively large number of objects provides a basis for studies of the physical processes involved and gives rise to speculations about possible relationships between comets and some amongst the Earth-approaching asteroids. Besides, far from the Sun, a comet may look stellar even when possessing a dust coma several thousand kilometers across. 


\subsection{EVOLUTION OF ACTIVE REGIONS AS AGING VENTS}

Little morphological information on the active regions has been provided by the closeup images of Halley's nucleus taken with cameras on the intercepting spacecraft (cf. Szegö's paper in these proceedings). The outlines, depth, wall texture, and general morphology of these regions will obviously remain controversial and conjectural until the time of implementation of a rendezvous mission to a comet. However, the results of the erosion rates for low-density comets suggest life spans of active regions that, for objects of short orbital periods, are comparable with the lengths of their observing records. Encke's comet, the most obvious choice for this type of investigation, orbits the Sun every $3.3 \mathrm{yr}$ and has already made more than 60 revolutions since its discovery in 1786. Its asymmetric coma, which is a product of outgassing from the active region on the comet's northern hemisphere (Source I in Fig. 8), has definitely been under observation ever since 1805 (cf. Whipple and Sekanina 1979 for original references), so that the region's minimum age is $\mathbf{5 5}$ revolutions, or about $180 \mathrm{yr}$. The equivalent depth of an eroded layer during this period of time amounts to some $1.4 \mathrm{~km}$ at a density of $0.2 \mathrm{~g} / \mathrm{cm}^{3}$, or approximately 50 percent of the nuclear radius (Sec. 5.3), and the corresponding diameter-to-depth ratio of the active region-based on its estimated cross-sectional area of $0.4 \mathrm{~km}^{2}$ (Sec. 4.3)-comes out to be 1:2! A depression of these dimensions can appropriately be described as a vent, a term used below interchangeably for an active region.

The question that can be answered in no certain terms is whether the conditions on the nucleus, especially the vent's response to the gradual increase in its depth, would allow this advanced stage of evolution ever to take place. The issues one faces here are those of the mechanical and morphological properties of the region and an interplay of the processes that bring about the vent's activation, gradual aging, dormancy, episodes of rejuvenation, and eventual deactivation or extinction. Plausible scenarios may involve the vent's partial wall collapse in the course of continuing outgassing, causing a new surface to be exposed, which-depending upon its history-may or may not be enriched in volatiles. The deposited debris can cut off sunlight from reaching some of the outgassing areas of the vent's surface, thus choking them up. The net effect on the post-collapse gas production from the vent must vary from case to case, with an increase or decrease with respect to the pre-collapse level being equally plausible. Perhaps it is this tug of war between the erosion and deposition processes under the conditions of microgravity and hostile radiation environment that can explain the puzzling, erratic variations in the vents' activity and that is responsible for the aging, rejuvenation, and slow migration of the emission centers over the nucleus.

Another source of uncertainty is the physical appearance of active regions and the interpretation of their cross-sectional areas (Sec. 5.2). It is entirely unclear whether these sources are single large cavities, or if they are made up of a complicated system of intertwined channels or interstitial crevices of various dimensions penetrating into the nucleus interior, or anything in between the two extremes. Some evidence for microstructure of the emission centers on Halley's nucleus is provided by Thomas and Keller's (1987) detection, with the Giotto's camera, of fine dust structures (filaments), many of them emanating from the major active regions and some intersecting each other very close to the nucleus. It is likely that appreciable depths of the active regions contribute to the observed high collimation of the dust and gas flows from many comets. Obviously, a complicated vertical structuring of a vent system could dramatically reduce its effective diameter-to-depth ratio compared with that of the single-cavity scenario. As a result, however, the floor's exposure 
to sunlight would be significantly restricted, causing the walls to account for virtually all the vent's emission and thus increasing its effective diameter-to-depth ratio. A successful empirical model, which represents dust jets in Halley's comet as products of emission from a continuous distribution of point sources making up an extended source and which explains the observed flattening of the jets' radial intensity profiles near the nucleus surface (Thomas et al. 1988; Huebner et al. 1988; Boice et al. 1988), is based on an assumption that ejection proceeds from undepressed surfaces, but each jet's cone angle is defined by a "hypothetical point source" that is located below the surface.

Perhaps the most difficult issue to address is that of how vents become activated. In the absence of better candidate mechanisms, the exposure of an icy reservoir by progressive thermal fracturing of a structurally weakened area of the overlying dust mantle can be offered as one possibility. Indeed, Kührt (1984) called attention to the important role that thermal stresses, caused by steep temperature gradients in the surface layer, play in cometary nuclei. He pointed out that they could be responsible for such phenomena as outbursts and nucleus splitting. Tauber and Kührt (1987) subsequently calculated thermal stresses in water-ice nuclei that contain various inclusions, finding them to bring about extensive cracks near the surface. Sekanina and Larson (1986a) showed that because of the large temperature differences $\left(100^{\circ} \mathrm{K}\right.$ or more at moderate and small heliocentric distances) between the sunlit surface of the mantled areas and their interface with the underlying reservoir of volatiles, the resulting thermal stresses could reach $10^{8} \mathrm{dyn} / \mathrm{cm}^{2}$ in the crust's uppermost layer, more than sufficient to produce an extensive network of superficial fractures even in a consolidated mantle.

Alternative mechanisms of vent activation that can be proposed are essentially those already considered in Sec. $\mathbf{4 . 4}$ for comet outbursts. For very irregular nuclei, one may also consider mechanical stresses caused by deformations due to complicated rotational motions. However, if mechanical stresses should be important on Halley's nucleus, one would expect preferential activation in the "waist" area of its surface. Instead, at least one of the major active regions is located not too far from the long end of the body.

Some of the possible evolutionary stages of vents on cometary nuclei are schematically represented in Fig. 9. Panel $a$ shows a section of the mantled surface of a comet nucleus, with the underlying dirty-ice reservoir. Following a fracture along lines of structural weakness, a vent is activated (panel $b$ ). While the mantle's fracturing process continues, a divergent flow of material from the shallow vent keeps maintaining a wide cone of debris (panel $c$ ). To a terrestrial observer, a comet with such shallow vents may display an essentially structureless dust coma. As time goes on, the vent's depth grows and the ejecta flow becomes more collimated (panel d). During periods of no exposure to the Sun (when it is outside the cone $\mathrm{AB}$ ), the vent becomes dormant (or nearly dormant, if the mantle's overhanging walls represent nontrivial sources of energy; panel $e$ ). If a sizable fragment of the mantle with an attached layer of ice breaks off and falls into the vent, it may block parts of the old cavity and expose a new and possibly larger active area (panel $f$ ). The rejuvenated vent migrates slowly, changes its outlines completely, and may lead to the formation of a near-vacuum bubble confined by the mantle's wall and fragments. Not shown in Fig. 9 are other scenarios, including one for an extinct vent-a cavity that runs out of ice. Considering the variety of possible combined situations, it is obvious that an extinct vent need not be very deep, as fragments of the mantle get easily dislodged, ending up with equal chance on the top or underneath an ice layer. Once the inert material covers the vent's new floor, it quenches (or at least reduces) its activity. Similarly, one can envision different types of vent dormancy: 


\section{EVOLUTION OF A VENT}

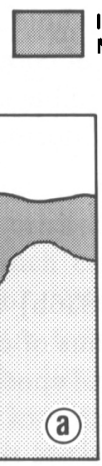

CONSOLIDATED MANTLE WITH UNDERLYING DIRTY ICE

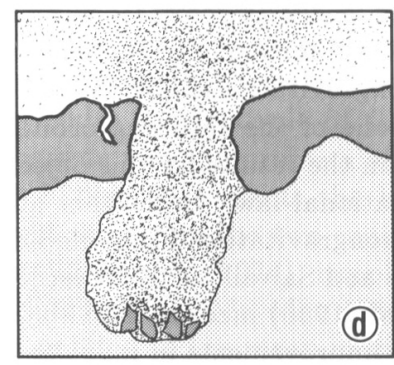

DEEP VENT (COLLIMATED FLOW)
INERT
MATERIAL

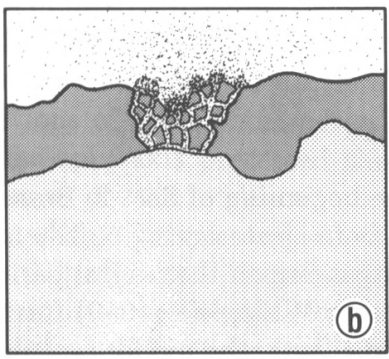

FRACTURED MANTLE

(VENT'S ACTIVATION)

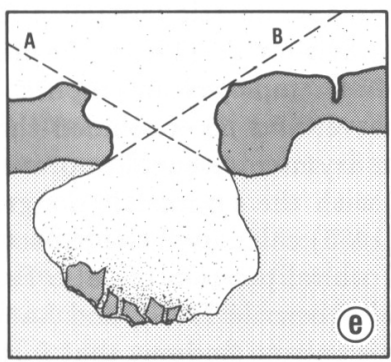

VENT'S

NEAR DORMANCY
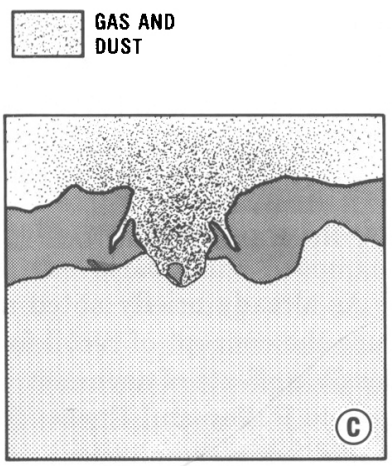

SHALLOW VENT (DIVERGENT FLOW)

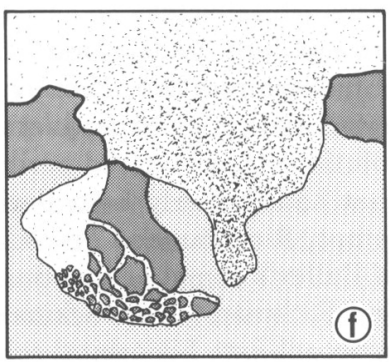

VENT'S

REJUVENATION/MIGRATION

Figure 9. Schematic representation of possible developments in the evolution of an active region on a cometary nucleus. See Sec. 5.4 for a description of the scenarios in the six panels and for discussion of their implications for the nucleus evolution.

one associated with the diurnal cycle, another with seasonal variations, yet another with morphological changes on the vent's walls and floor, etc.

Thus, three types of vents can generally be distinguished on comet nuclei: active (which include rejuvenated ones), dormant, and extinct. The active vents can be of very different ages and depths. Since the diameter-to-depth ratio affects the degree of jet collimation, then whether or not a dust-rich comet exhibits jets in its head should depend primarily upon the age of its vents - a conclusion based on a presumed correlation between the collimation of jets and their sharpness on the ground-based images of comets. In other words, the failure of terrestrial observers to detect jets does not necessarily imply the absence of discrete active regions on the comet's nucleus.

From the results of this section, it becomes increasingly obvious that the evolution of an active region in one part of the nucleus surface is largely independent of the evolution of another active region at a different location on the surface. The only global-scale process that could provide "communication" among individual discrete sources is random 
deposition-with the possibility of a gradual regolith-type buildup-of fine dust grains entrained in the surface breeze of the circumnuclear pressure-equalization gas flow (Sec. 2.3). Unless this process plays a major role in the surface evolution, attempts to describe the aging of comets in terms of characteristics of the nucleus as a whole are too simplistic for objects with discrete active centers. The traditional perception of cometary deactivation as a slow, monotonic process that concludes with an object's irreversible extinction needs to be replaced with a more dynamic concept of intermittent periods of dormancy and reactivation of the localized emission sources.

\subsection{NONGRAVITATIONAL EFFECTS}

As already briefly noted at the beginning of Sec. 2, Bessel (1836b) was the first to introduce the concept of recoil forces from ejecta acting on the motions of comets and to discuss possible effects of asymmetric outgassing on the orbital periods of specific objects, including comets Halley and Encke. Following Whipple's (1950) formulation of his icy conglomerate model, which emphasizes rotation effects, the modern, sophisticated techniques of accounting for the nongravitational effects in the equations of cometary motion concentrated on symmetric models (Marsden 1969). Although tested, in particular, on Encke's comet, the early orbital solutions based on an asymmetric law failed (Marsden 1970) for reasons that are not fully understood. Using Sekanina's (1981a) formalism to account for the effects of nuclear rotation, Yeomans (1984) employed an empirical nongravitational law that followed Halley's gas-production curve, and he concluded that none of the resulting orbital solutions that involved perihelion asymmetry could improve upon the results for this object that had formerly been derived with the symmetric nongravitational law. In another attempt, Rickman and Froeschlé (1983) calculated the expected nongravitational parameters of Halley's comet as functions of orbital position (cf. also Fanale and Salvail 1986; Froeschlé and Rickman 1986; Rickman and Froeschlé 1986), and Landgraf (1986) incorporated their approach into his orbital solutions only to find out that further improvements in modelling the nongravitational forces were necessary. Sekanina's (1988c) motivation for employing an asymmetric nongravitational law was based on his perception of major seasonal effects due to a highly nonrandom distribution of active regions on the nucleus surface and on the observed statistical correlation between the perihelion asymmetry of the available indicators of cometary activity (usually the light curve) and the sense of the nongravitational effect (acceleration versus deceleration). He suggested that at the cost of a potentially significant loss of generality, a form of the asymmetric law can be prescribed by applying the standard law, in use now for almost two decades (cf. Marsden, Sekanina, and Yeomans 1973), in which heliocentric distance is taken not at the observation time $t$, but at a time $t-\tau$. The nongravitational force then peaks either before perihelion if $\tau<0$, or after perihelion if $\tau>0$. Significant progress with this asymmetric model seems now to have been achieved (Yeomans and Chodas 1989), even though work on this project is still continuing at the time of this writing and the new approach has not yet been applied to a number of comets, including Encke's.

Progress has also been slow in the understanding of the physical aspects of the nongravitational forces. Although the basic quantity determined from both Bessel's and Whipple's concepts is the comet's mass-loss rate, the interpretations of the observed long-term variations in the mean motion have so far been inconclusive. The classical relationship between the sense of nuclear rotation and the sense of the nongravitational effect (with a deceleration 
of the mean motion implying a direct sense of rotation with respect to the comet's orbital motion and vice versa), based on the assumption that the effect is due to the transverse component of the force, is obviously invalid when the contribution by the asymmetric radial component of the force dominates. A decrease of the nongravitational perturbation with time found for a number of short-period comets, including Encke's since the early 19th century, was first explained in terms of progressive deactivation (Marsden 1969; Sekanina 1969, 1972a), leading to the postulation of a core-mantle model for the nuclei of these comets. Transitions from nongravitational acceleration to deceleration and vice versa, which were soon detected in the orbital motions of other short-period comets, were interpreted to indicate that in each such case the spin axis precessed through the orbit plane (Yeomans 1971; Sekanina 1972b). With more of this evidence subsequently forthcoming, elaborate precession models were formulated for Encke's and other comets (Whipple and Sekanina 1979; Sekanina 1984b, 1985a,b; Sekanina and Yeomans 1985). The renewed emphasis on the perihelion asymmetry's contribution to the nongravitational effect in the mean motion (Rickman 1986, 1989; Rickman et al. 1987; Sekanina 1988c) once again upset the status quo. In particular, Yeomans and Chodas (1989) found that application of the new, asymmetric law has a dramatic effect on the values of the nongravitational parameters. Kamél (1989) argued that the nongravitational effects on Encke's nucleus are nowadays so small because the perihelion asymmetry of the light curve and gas production has been decreasing. Although an obvious oversimplification that Kamél admits, this kind of argumentation provides a useful rule of thumb that can, with caution, be employed to gain remarkable insights into the problem of the nongravitational perturbations.

Figure 10 presents the history of the nongravitational perturbations of Encke's mean motion during the two centuries of observation. The data points are taken from Marsden and Sekanina (1974), supplemented with information on the apparitions since 1971 from other sources (Marsden 1986; Nakano 1987). The results are from the symmetric solutions, converted to the mean-motion effect by using an appropriate transformation formula. The time coordinate plotted is the middle of the span employed to derive the nongravitational parameters, which, in most cases, was five consecutive apparitions, or about 13-14 yr. In comparison with the nongravitational accelerations of other short-period comets, that of Encke's comet is known to have been changing fairly smoothly with time. However, Marsden and Sekanina (1974) commented on considerable difficulties they experienced with the orbital solutions that included the apparitions of 1868 and 1898 . Using the above rule of thumb, the discontinuity in the 1890s suggests that the preperihelion brightness excess diminished by a measurable amount at that time. Since the preperihelion activity is associated with the north hemisphere's source and the activity just after perihelion with the south hemisphere's source (Sec. 4.3), the discontinuity can be interpreted to be due to the birth of the southern vent. A sunward extension of Encke's coma associated with this source appears to have been first detected visually by Jones (1985) in 1961 (cf. Sekanina 1988 b), at which time the vent should have been some 20 revolutions old and, from Fig. 8 , over $600 \mathrm{~m}$ deep at the assumed density of $0.2 \mathrm{~g} / \mathrm{cm}^{3}$. Minor discontinuities may also have taken place in the 1830 s and possibly even very recently, during the past $20 \mathrm{yr}$.

If precession and other effects (such as the potential perihelion asymmetry of the transferred momentum or the discrepancy between the light curve and the water-production curve; cf. Sec. 4.3) are ignored, one can similarly interpret the additional aspects of the curve in Fig. 10. Of particular interest is the early increase in the nongravitational effect, which implies a strongly increasing perihelion asymmetry and therefore a rapidly expand- 
ing area of the north hemisphere's vent, whose existence was documented as early as 1805 (Sec. 5.4). Extrapolating back in time prior to 1786, one can estimate that the northern vent may have become activated as late as $\sim 1750$ or even later and that, quite conceivably, the whole nucleus of Encke's comet may have been dormant for an unknown period of time before then. It is most fitting to mention that Whipple and Hamid's (1972) intensive search for Encke's comet among nearly 600 transient objects in Ho's (1962) catalog, which covers the period of time until A.D. 1600 , turned up no definite candidate and only a handful of suspects. It is distinctly possible that Encke's comet was dormant over extensive periods of time in the past after having produced the Taurid meteor complex several thousand years ago and that the most recent of these dormant phases came to an end during the 18th century. Looking ahead, it is apparent from Fig. 10 that-barring another major discontinuity in the sense opposite to that of the 1890s-Encke's nongravitational acceleration will turn into a deceleration in the 1990s and that-following the termination of outgassing from the current north hemisphere's vent-the comet's pattern of activity is likely to change dramatically in the 21 st century.

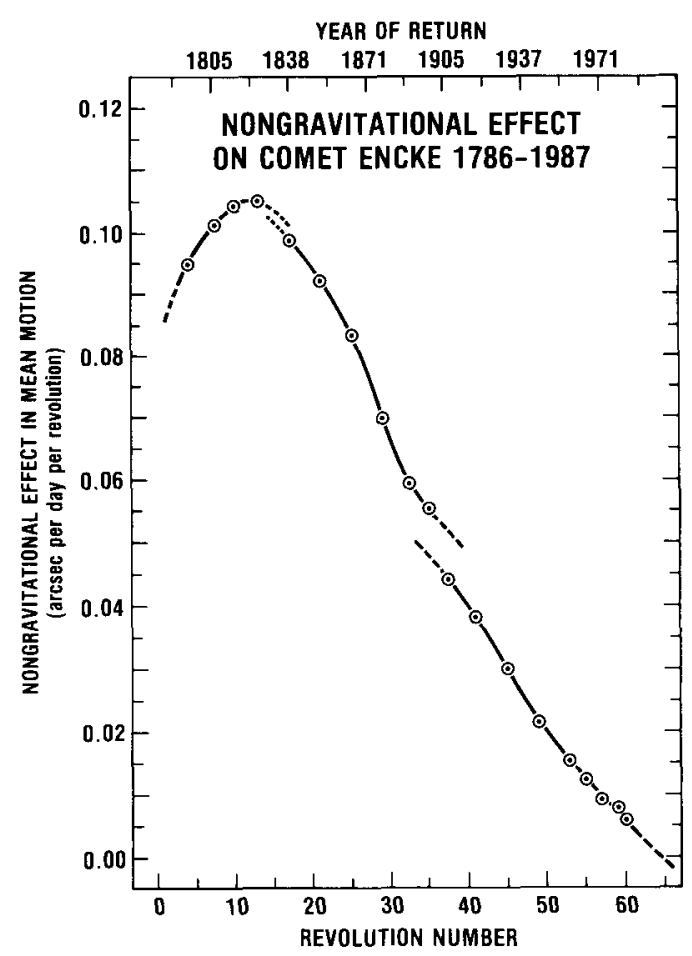

Fig. 10. Nongravitational perturbation of Encke's mean orbital motion between 1786 and 1987. The dashed parts of the curve are extrapolations, some of which are intended to emphasize the magnitude of the discontinuities. See Sec. 5.5 for a discussion and interpretation of the curve. (Adapted from Marsden and Sekanina 1974 and updated with the data from Marsden 1986 and Nakano 1987.)

In the same context, the decrease of Encke's nongravitational acceleration since the early 19 th century can be understood as a gradual reduction of the outgassing area of the north hemisphere's vent, but, in the period since the $1890 \mathrm{~s}$, also, alternatively, as a gradual increase in the outgassing area of the south hemisphere's vent, or a combination of the two developments. The reported poleward migration of the northern vent's southern boundary 
during the last 100 years or so (Sekanina 1988c) may signal the vent's retreat and progressive deactivation. On the other hand, the southern vent's outgassing area may still be growing larger at the present time, if the sole 1987 data point in Fig. 5 is taken at face value. This obviously needs confirmation at the comet's future returns to the Sun.

Discontinuities in the perturbations of Encke's mean motion caused by the nongravitational force can also be detected as sudden changes in the slope of the curve of residuals that the observed times of perihelion passage give with respect to a prescribed smooth law when plotted as a function of the revolution number. For example, Sitarski's (1987) polynomial fit (including a cubic term) to the nongravitational variations in the comet's semimajor axis produces the residuals plotted in Fig. 11, where a few sharp slope turnovers are readily apparent. Following the poorly defined early portion of the curve (Sections $\mathrm{Ia}-\mathrm{Ib}$ ), the slopes are virtually constant in the course of the time periods identified as Sections II, III, IVa-b, and V. Major events are therefore suspected to have occurred at the beginning of the 19th century, about 1850 , in the late $1860 \mathrm{~s}$, and at the beginning of the 20th century. Another, minor event just before 1890 is doubtful. The slopes of the curve of residuals are unimportant, because they depend primarily upon the choice of the fitting law. Unfortunately, some of the resulting slope turnovers can be artifacts of the fitting law used, so that caution ought to be exercised in the interpretation. At least two correlations are clearly indicated:

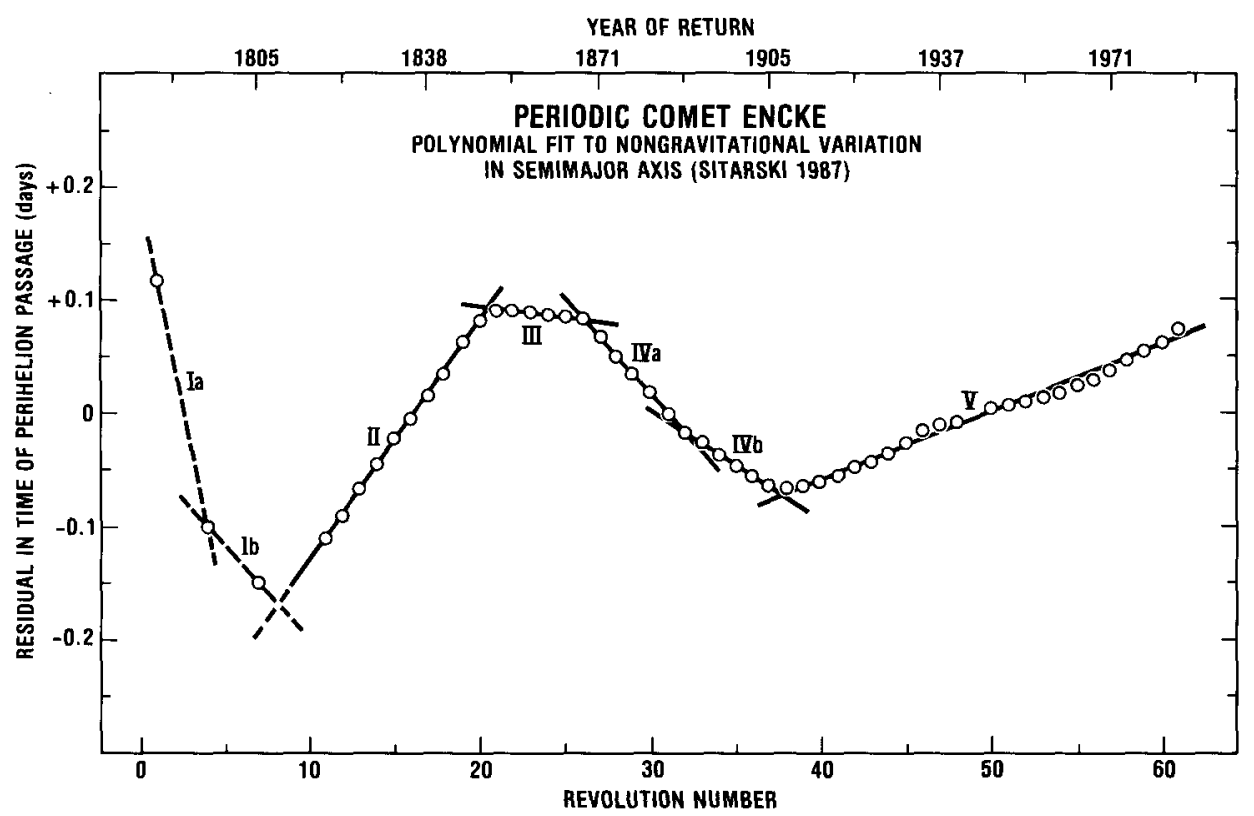

Figure 11. Temporal variations in the residuals between the observed times of perihelion passage of Encke's comet and their calculated values from Sitarski's (1987) cubic fit to the nongravitational effects in the comet's semimajor axis. The curve can be divided into several sections, identified by the roman numerals, within which the residuals can be fitted by straight lines. Sharp changes in the slope between neighboring sections could be associated with discontinuities in the nongravitational perturbations of Encke's orbital motion, even though artifacts of the fitting law may also be involved. 
(1) between the discontinuity in the 1890 s in Fig. 10 and the beginning of Section V in Fig. 11, and (2) between the troublesome orbital solutions involving 1868, as reported by Marsden and Sekanina (1974), and the beginning of Section IVa. Less obvious is a possible correlation between the minor discontinuity in the 1830s and the beginning of Section III.

Depending largely upon the nucleus shape, size, and spin rate, precession effects cannot, of course, be generally ignored, even though in the case of comet Encke they appear to have amounted to very little recently (Sekanina 1988b,c). Other factors-such as the momentumtransfer efficiency, which may correlate with the degree of flow collimation-should also affect the magnitude of the nongravitational force. Mixed evidence is apparent from Table 4. On the one hand, the two short-period comets with the largest outgassing fractions of the nucleus surface happen to exhibit sizable nongravitational perturbations that changed in the recent past from a deceleration to an acceleration (cf. Marsden 1986) and the motions of the two comets of very low activity-Arend-Rigaux and Neujmin 1-comply with the gravitational law (e.g., Marsden 1972). On the other hand, Halley's comet, which also is among the most active objects in Table 4, is known to have had remarkably constant nongravitational perturbations during the past two millennia (cf. Yeomans and Kiang 1981). In any case, it appears likely that the erratic discontinuities in the nongravitational meanmotion perturbations - exemplified by the events experienced by periodic comets GiacobiniZinner, Brorsen, Forbes, Tuttle-Giacobini-Kresák, Finlay, and others-signal activation of major new sources and/or sudden extinction of once active vents.

\section{Future Research}

Considerable advances have been achieved in the interpretation of the observed dust-coma morphology and other signatures of the physical processes of activation and extinction of discrete emission sources on cometary nuclei, which provide basic clues to the understanding of the nucleus-surface evolution. Yet the processes themselves are still elusive and major efforts will have to be expended to learn more about their characteristics. While dedicated rendezvous missions to comets are likely to offer a lion's share of new information in the more distant future, ground-based, aircraft, and near-Earth observations and their synergistic studies along the lines already pursued in the past should continue to provide new results for the rest of this century and a balance of important information in the long run.

In terms of the objects of interest, diversity is most desirable. In addition to Halley's comet, whose observations will still be intensively analyzed during the forthcoming decade, the thrust of near-term research should concentrate on three kinds of objects: (1) shortperiod comets with long histories of observation (such as Encke, d'Arrest, Faye, PonsWinnecke, and Tempel 2); (2) comets that have experienced a close encounter with Earth, because-statistically - the prominence of coma structure and detail detectable on the nucleus is correlated inversely with geocentric distance (for some techniques, such as the radar experiments, with its high power); and (3) peculiar objects, with unusual light curves, morphology, or other diagnostic signatures that could be interpreted in terms of major events occurring on these comets' nuclei on short time scales. It is hoped that integrated results of such studies will contribute to further advancements in our quest to understand the dust-coma morphology and nucleus activity, an area of investigation that--along with other branches of cometary physics-has reached a new, qualitatively higher level of insight and sophistication in the post-Halley era. 


\section{Acknowledgments}

I thank S.M. Larson, C.-Y. Shao, and H. Spinrad for kindly providing high-quality copies of their photographic images of comets Bennett, Schwassmann-Wachmann 1, and Encke that appear in Fig. 1. I also thank M.F. A'Hearn, S.M. Larson, B.G. Marsden, and R.L. Newburn, Jr., for reading the manuscript and offering helpful comments. Several colleagues kindly sent their manuscripts before publication. The research described in this paper was carried out by the Jet Propulsion Laboratory, California Institute of Technology, under contract with the National Aeronautics and Space Administration.

\section{References}

A'Hearn, M.F. (1988) 'Observations of cometary nuclei', Ann. Rev. Earth Planet. Sci. 16, 272-293.

A'Hearn, M.F., Birch, P.V., Feldman, P.D., and Millis, R.L. (1985) 'Comet Encke: Gas production and lightcurve', Icarus 64, 1-10.

A'Hearn, M.F., Campins, H., Schleicher, D.G., and Millis, R.L. (1989) 'The nucleus of comet P/Tempel 2', Astrophys. J. 347, 1155-1166.

A'Hearn, M.F., Hoban, S., Birch, P.V., Bowers, C., Martin, R., and Klinglesmith, D.A. (1986a) 'CN jets in comet $\mathrm{P} /$ Halley', Nature 324, 649-651.

A'Hearn, M.F., Hoban, S., Birch, P.V., Bowers, C., Martin, R., and Klinglesmith, D.A. (1986b) 'Gaseous jets in comet P/Halley', in B. Battrick, E.J. Rolfe, and R. Reinhard (eds.), Exploration of Halley's Comet, ESA SP-250, ESTEC, Noordwijk, vol. 1, pp. 483486.

A'Hearn, M.F., Millis, R.L., and Thompson, D.T. (1983) 'The disappearance of OH from comet $\mathrm{P} /$ Encke', Icarus 55, 250-258.

A'Hearn, M.F., and Schleicher, D.G. (1988) 'Comet P/Encke's nongravitational force', Astrophys. J. 331, L47-L51.

Bertaux, J.L., Blamont, J.E., and Festou, M.C. (1973) 'Interpretation of hydrogen Lymanalpha observations of comets Bennett and Encke', Astron. Astrophys. 25, 415-430.

Beisser, K., and Boehnhardt, H. (1987) 'Evidence for the nucleus rotation in streamer patterns of comet Halley's dust tail', Astrophys. Space Sci. 139, 5-12.

Bessel, F.W. (1836a) 'Beobachtungen über die physische Beschaffenheit des Halleyschen Kometen und dadurch veranlasste Bemerkungen', Astron. Nachr. 13, 185-232.

Bessel, F.W. (1836b) 'Bemerkungen über mögliche Unzulänglichkeit der die Anziehungen allein berücksichtigenden Theorie der Kometen', Astron. Nachr. 13, 345-350.

Beyer, M. (1962) 'Physische Beobachtungen von Kometen. XII', Astron. Nachr. 286, 219240.

Bobrovnikoff, N.T. (1931) 'Halley's comet in its apparition of 1909-1911', Publ. Lick Obs. 17, 305-482.

Bobrovnikoff, N.T. (1954) 'Physical properties of comets', Astron. J. 59, 357-358.

Bockelée-Morvan, D., Crovisier, J., Gérard, E., and Kazès, I. (1981) 'Observations of the $\mathrm{OH}$ radical in comets at $18 \mathrm{~cm}$ wavelength', Icarus 47, 464-469.

Bockelée-Morvan, D., and Gérard, E. (1984) 'Radio observations of the hydroxyl radical in comets with high spectral resolution. Kinematics and asymmetries of the $\mathrm{OH}$ coma in C/Meier (1978 XXI), C/Bradfield (1979 X), and C/Austin (1982g)', Astron. Astrophys. 131, 111-122. 
Boice, D.C., Huebner, W.F., Reitsema, H.J., and Delamere, W.A. (1988) 'A model of the near-nucleus dust distribution of comet $\mathrm{P} / \mathrm{Halley}$ : The effects of spacecraft viewing geometry and surface curvature', Bull. Am. Astron. Soc. 20, 826. (Abstract.)

Bond, G.P. (1862) 'Account of the great comet of 1858', Ann. Harvard Coll. Obs. 3, 1-372.

Brin, G.D. (1980) 'Three models of dust layers on cometary nuclei', Astrophys. J. 237, 265-279.

Brin, G.D., and Mendis, D.A. (1979) 'Dust release and mantle development in comets', Astrophys. J. 229, 402-408.

Campbell, D.B., Harmon, J.K., and Shapiro, I.I. (1989) 'Radar observations of comet Halley', Astrophys. J. 338, 1094-1105.

Campins, H. (1988) 'The anomalous dust production in periodic comet Encke', Icarus 73, 580-587.

Campins, H., A'Hearn, M.F., and McFadden, L.-A. (1987) 'The bare nucleus of comet Neujmin 1', Astrophys. J. 316, 847-857.

Campins, H., Joy, M., Harvey, P.M., Lester, D.F., and Ellis, H.B., Jr. (1987a) 'Photometry of comet P/Halley from 40 to 160 microns', Astron. Astrophys. 187, 632-634.

Campins, H., Telesco, C.M., Decher, R., and Ramsey, B.D. (1987b) 'Thermal infrared imaging of comet P/Halley', Astron. Astrophys. 187, 601-604.

Carey, W.C., McDonnell, J.A.M., Welch, C.S., and Zarnecki, J.C. (1984) 'Heterogeneous grain morphologies and acceleration mechanisms in cometary coma dust dynamics: Mass envelope dispersion', Adv. Space Res. 4, No. 9, pp. 217-220.

Ceplecha, Z. (1986) 'Photographic fireball networks', in C.-I. Lagerkvist, B.A. Lindblad, H. Lundstedt, and H. Rickman (eds.), Asteroids, Comets, Meteors II, University of Uppsala, Uppsala, pp. 575-582.

Chakaveh, S.C., Green, S.F., Ridley, J.K., McDonnell, J.A.M., and Hughes, D.W. (1986) 'Computer modelling of dust from comet $\mathrm{P} / \mathrm{Halley}$ and comparison with ground-based observations and in situ measurements', in B. Battrick, E.J. Rolfe, and R. Reinhard (eds.), Exploration of Halley's Comet, ESA SP-250, ESTEC, Noordwijk, vol. 2, pp. 163-165.

Chofardet, P. (1923) 'Observations de planètes et de la comète Tempel II (1920a)', Astron. $J .34,33-36$.

Cochran, A.L., Barker, E.S., and Cochran, W.D. (1980) 'Spectrophotometric observations of P/Schwassmann-Wachmann 1 during outburst', Astron. J. 85, 474-477.

Cochran, A.L., Cochran, W.D., and Barker, E.S. (1982) 'Spectrophotometry of comet Schwassmann-Wachmann 1. II. Its color and $\mathrm{CO}^{+}$emission', Astrophys. J. 254, 816822.

Colwell, J.E., and Jakosky, B.M. (1987) 'The evolution of topography on a comet', Icarus $72,128-134$.

Combi, M.R. (1987) 'Sources of cometary radicals and their jets: Gases or grains', Icarus 71, 178-191.

Combi, M.R., Stewart, A.I.F., and Smyth, W.H. (1984) 'An analysis of Pioneer Venus hydrogen Lyman- $\alpha$ observations of comet P/Encke', Bull. Am. Astron. Soc. 16, 638. (Abstract.)

Cordes, J., Falchi, A., Tofani, G., Lewis, B.M., and Terzian, Y. (1986) 'Radio OH observation of Halley's comet', in B. Battrick, E.J. Rolfe, and R. Reinhard (eds.), Exploration of Halley's Comet, ESA SP-250, ESTEC, Noordwijk, vol. 3, pp. 113-115.

Cosmovici, C.B., Green, S.F., Hughes, D.W., Keller, H.U., Mack, P., Moreno-Insertis, F., and Schmidt, H.U. (1986) 'Groundbased CCD observations of comet Halley with the 
Giotto HMC filters', in B. Battrick, E.J. Rolfe, and R. Reinhard (eds.), Exploration of Halley's Comet, ESA SP-250, ESTEC, Noordwijk, vol. 2, pp. 375-379.

Cosmovici, C.B., Mack, P., Schwarz, G., Craubner, A., and Ip, W.-H. (1987) 'CCD observations of gas and dust jets in the coma of comet Halley', in E.J. Rolfe and B. Battrick (eds.), Diversity and Similarity of Comets, ESA SP-278, ESTEC, Noordwijk, pp. 195-207.

Cosmovici, C.B., Schwarz, G., Ip, W.-H., and Mack, P. (1988) 'Gas and dust jets in the inner coma of comet Halley', Nature 332, 705-709.

Cowan, J.J., and A'Hearn, M.F. (1979) 'Vaporization of comet nuclei: Light curves and lifetimes', Moon Planets 21, 155-171.

Crifo, J.F. (1988) 'Cometary dust sizing: Comparison between optical and in situ sampling techniques', Part. Charact. 5, 38-46.

Crovisier, J. (1984) 'The water molecule in comets: Fluorescence mechanisms and thermodynamics of the inner coma', Astron. Astrophys. 130, 361-372.

Daniels, P.A., and Hughes, D.W. (1981) 'The accretion of cosmic dust-a computer simulation', Mon. Not. R. Astron. Soc. 195, 1001-1009.

Delsemme, A.H. (1982) 'Chemical composition of cometary nuclei', in L.L. Wilkening (ed.), Comets, University of Arizona, Tucson, pp. 85-130.

Delsemme, A.H., and Rud, D.A. (1973) 'Albedos and cross-sections of the nuclei of comets 1969 IX, 1970 II, and 1971 II', Astron. Astrophys. 28, 1-6.

de Pater, I., Palmer, P., and Snyder, L.E. (1986) 'The brightness distribution of OH around comet Halley', Astrophys. J. 304, L33-L36.

de Pater, I., Palmer, P., Snyder, L.E., and Ip, W.-H. (1986) 'VLA observations of comet Halley: The brightness distribution of $\mathrm{OH}$ around the comet', in B. Battrick, E.J. Rolfe, and R. Reinhard (eds.), Exploration of Halley's Comet, ESA SP-250, ESTEC, Noordwijk, vol. 1, pp. 409-412.

Divine, N., and Newburn, R.L., Jr. (1983) 'Numerical models for cometary dust environments', in T.I. Gombosi (ed.), Cometary Exploration, Hungarian Academy of Sciences, Budapest, vol. 2, pp. 81-98.

Djorgovski, S., and Spinrad, H. (1985) 'Surface photometry of comet P/Encke', Astron. J. $90,869-876$.

Donn, B. (1963) 'The origin and structure of icy cometary nuclei', Icarus 2, 396-402.

Donn, B. (1976) 'The nucleus: Panel discussion', in B. Donn, M. Mumma, W. Jackson, M. A'Hearn, and R. Harrington (eds.), The Study of Comets, NASA SP-393, U.S. GPO, Washington, D.C., pp. 611-619.

Donn, B., Daniels, P.A., and Hughes, D.W. (1985) 'On the structure of the cometary nucleus', Bull. Am. Astron. Soc. 17, 520. (Abstract.)

Donn, B., and Hughes, D.W. (1986) 'A fractal model of a cometary nucleus formed by random accretion', in B. Battrick, E.J. Rolfe, and R. Reinhard (eds.), Exploration of Halley's Comet, ESA SP-250, ESTEC, Noordwijk, vol. 3, pp. 523-524.

Donn, B., and Urey, H.C. (1956) 'On the mechanism of comet outbursts and the chemical composition of comets', Astrophys. J. 123, 339-342.

Dubiago, A.D. (1948) 'On the secular acceleration of motions of periodic comets', Astron. J. USSR 25, 361-368. (In Russian.)

Edenhofer, P., Bird, M.K., Brenkle, J.P., Buschert, H., Kursinski, E.R., Mottinger, N.A., Porsche, H., Stelzried, C.T., and Volland, H. (1987) 'Dust distribution of comet P/Halley's inner coma determined from the Giotto Radio-Science Experiment', Astron. Astrophys. 187, 712-718. 
Fanale, F.P., and Salvail, J.R. (1984) 'An idealized short-period comet model: Surface insolation, $\mathrm{H}_{2} \mathrm{O}$ flux, dust flux, and mantle evolution', Icarus 60, 476-511.

Fanale, F.P., and Salvail, J.R. (1986) 'A model of cometary gas and dust production and nongravitational forces with application to $\mathrm{P} /$ Halley', Icarus 66, 154-164.

Feldman, P.D., A'Hearn, M.F., Festou, M.C., McFadden, L.A., Weaver, H.A., and Woods, T.N. (1986) 'Is $\mathrm{CO}_{2}$ responsible for the outbursts of comet Halley?', Nature 324, 433-436.

Feldman, P.D., A'Hearn, M.F., and Millis, R.L. (1984) 'Temporal and spatial behavior of the ultraviolet emissions of comet IRAS-Araki-Alcock 1983d', Astrophys. J. 282, 799-802.

Feldman, P.D., Festou, M.C., A'Hearn, M.F., Arpigny, C., Butterworth, P.S., Cosmovici, C.B., Danks, A.C., Gilmozzi, R., Jackson, W.M., McFadden, L.A., Patriarchi, P., Schleicher, D.G., Tozzi, G.P., Wallis, M.K., Weaver, H.A., and Woods, T.N. (1987) 'IUE observations of comet P/Halley: Evolution of the ultraviolet spectrum between September 1985 and July 1986', Astron. Astrophys. 187, 325-328.

Feldman, P.D., Weaver, H.A., and Festou, M.C. (1984) 'The ultraviolet spectrum of periodic comet Encke (1980 XI)', Icarus 60, 455-463.

Fertig, J., and Schwehm, G.H. (1984) 'Dust environment models for comet P/Halley: Support for targeting of the Giotto S/C', Adv. Space Res. 4, No. 9, pp. 213-216.

Festou, M.C., Feldman, P.D., A'Hearn, M.F., Arpigny, C., Cosmovici, C.B., Danks, A.C., McFadden, L.A., Gilmozzi, R., Patriarchi, P., Tozzi, G.P., Wallis, M.K., and Weaver, H.A. (1986) 'IUE observations of comet Halley during the VEGA and Giotto encounters', Nature 321, 361-363.

Finson, M.L., and Probstein, R.F. (1968) 'A theory of dust comets. I. Model and equations', Astrophys. J. 154, 327-352.

Froeschlé, C., and Rickman, H. (1986) 'Model calculations of nongravitational forces on short-period comets. I. Low-obliquity case', Astron. Astrophys. 170, 145-160.

Gehrz, R.D., and Ney, E.P. (1986) 'Infrared temporal development of P/Halley', in B. Battrick, E.J. Rolfe, and R. Reinhard (eds.), Exploration of Halley's Comet, ESA SP-250, ESTEC, Noordwijk, vol. 2, pp. 101-105.

Goldstein, R.M., Jurgens, R.F., and Sekanina, Z. (1984) 'A radar study of comet IRASAraki-Alcock 1983d', Astron. J. 89, 1745-1754.

Gombosi, T.I., and Horányi, M. (1986a) 'Modeling of dust halo formation following comet outbursts: Preliminary results', Geophys. Res. Lett. 13, 299-301.

Gombosi, T.I., and Horányi, M. (1986b) 'Time-dependent numerical modeling of dust halo formation at comets', Astrophys. J. 311, 491-500.

Gombosi, T.I., and Houpis, H.L.F. (1986) 'An icy-glue model of cometary nuclei', Nature 324, 43-44.

Greenberg, J.M. (1986) 'Fluffy comets', in C.-I. Lagerkvist, B.A. Lindblad, H. Lundstedt, and H. Rickman (eds.), Asteroids, Comets, Meteors II, University of Uppsala, Uppsala, pp. 221-223.

Greenstein, J.L. (1958) 'High-resolution spectra of comet Mrkos (1957d)', Astrophys. J. 128, 106-113.

Grün, E., Benkhoff, J., Fechtig, H., Hesselbarth, P., Klinger, J., Kochan, H., Kohl, H., Krankowsky, D., Lämmerzahl, P., Seboldt, W., Spohn, T., and Thiel, K. (1989) 'Mechanisms of dust emission from the surface of a cometary nucleus', Adv. Space Res. 9, No. 3, pp. 133-137.

Grün, E., Graser, U., Kohoutek, L., Thiele, U., Massonne, L., and Schwehm, G. (1986) 'Structures in the coma of comet Halley', Nature 321, 144-147. 
Grün, E., Kochan, H., Roessler, K., and Stöffler, D. (1987) 'Simulation of cometary nuclei', in E.J. Rolfe and B. Battrick (eds.), Diversity and Similarity of Comets, ESA SP-278, ESTEC, Noordwijk, pp. 501-508.

Grün, E., Massonne, L., and Schwehm, G. (1987) 'New properties of cometary dust', in E.J. Rolfe and B. Battrick (eds.), Diversity and Similarity of Comets, ESA SP-278, ESTEC, Noordwijk, pp. 305-314.

Hanner, M.S., Aitken, D.K., Knacke, R., McCorkle, S., Roche, P.F., and Tokunaga, A.T. (1985) 'Infrared spectrophotometry of comet IRAS-Araki-Alcock (1983d): A bare nucleus revealed?', Icarus 62, 97-109.

Hanner, M.S., and Newburn, R.L., Jr. (1989) 'Infrared photometry of comet Wilson (19861) at two epochs', Astron. J. 97, 254-261.

Hanner, M.S., Newburn, R.L., Jr., Spinrad, H., and Veeder, G.J. (1987a) 'Comet SuganoSaigusa-Fujikawa (1983 V)-a small, puzzling comet', Astron. J. 94, 1081-1087.

Hanner, M.S., Tokunaga, A.T., Golisch, W.F., Griep, D.M., and Kaminski, C.D. (1987b) 'Infrared emission from P/Halley's dust coma during March 1986', Astron. Astrophys. 187, 653-660.

Harmon, J.K., Campbell, D.B., Hine, A.A., Shapiro, I.I., and Marsden, B.G. (1989) 'Radar observations of comet IRAS-Araki-Alcock 1983d', Astrophys. J. 338, 1071-1093.

Hartmann, W.K., and Cruikshank, D.P. (1984) 'Comet color changes with solar distance', Icarus 57, 55-62.

Hartmann, W.K., Cruikshank, D.P., and Degewij, J. (1982) 'Remote comets and related bodies: VJHK colorimetry and surface materials', Icarus 52, 377-408.

Hartmann, W.K., Tholen, D.J., and Cruikshank, D.P. (1987) 'The relationship of active comets, "extinct" comets, and dark asteroids', Icarus 69, 33-50.

Harwit, M. (1968) ' "Spontaneously" split comets', Astrophys. J. 151, 789-790.

Hevelius, J. (1682) 'Excerpta ex epistola. II. De cometa anno 1682 mense Augusto \& Septimbri viso', Acta Erudit., Dec. 1682, pp. 389-391.

Hoban, S., A'Hearn, M.F., Birch, P.V., and Martin, R. (1989) 'Spatial structure in the color of the dust coma of comet P/Halley', Icarus 79, 145-158.

Hoban, S., Samarasinha, N.H., A'Hearn, M.F., and Klinglesmith, D.A. (1988) 'An investigation into periodicities in the morphology of $\mathrm{CN}$ jets in comet $\mathrm{P} / \mathrm{Halley}$ ', Astron. Astrophys. 195, 331-337.

Ho Peng Yoke (1962) 'Ancient and mediaeval observations of comets and novae in Chinese sources', Vistas Astron. 5, 127-225.

Horányi, M., and Mendis, D.A. (1985) 'Trajectories of charged dust grains in the cometary environment', Astrophys. J. 294, 357-368.

Horányi, M., and Mendis, D.A. (1986) 'The effects of electrostatic charging on the dust distribution at Halley's comet', Astrophys. J. 307, 800-807.

Huebner, W.F. (1975) 'On the structure of the nucleus of comet Kohoutek (1973f)', in G.A. Gary (ed.), Comet Kohoutek, NASA SP-355, U.S. GPO, Washington, D.C., pp. 145-151.

Huebner, W.F., Boice, D.C., Reitsema, H.J., and Delamere, W.A. (1988) 'A model for intensity profiles of dust jets near the nucleus of comet Halley', Icarus 76, 78-88.

Huebner, W.F., Keller, H.U., Wilhelm, K., Whipple, F.L., Delamere, W.A., Reitsema, H.J., and Schmidt, H.U. (1986) 'Dust-gas interaction deduced from Halley Multicolor Camera observations', in B. Battrick, E.J. Rolfe, and R. Reinhard (eds.), Exploration of Halley's Comet, ESA SP-250, ESTEC, Noordwijk, vol. 2, pp. 363-364. 
Ibadinov, Kh.I. (1989) 'Laboratory investigation of the sublimation of comet nucleus models', Adv. Space Res. 9, No. 3, pp. 97-112.

Ibadinov, Kh.I., Aliev, S.A., and Rahmonov, A.A. (1987) 'Physical-mechanical properties of matrices on the comet nuclei surface models', in E.J. Rolfe and B. Battrick (eds.), Diversity and Similarity of Comets, ESA SP-278, ESTEC, Noordwijk, pp. 713-716.

Ip, W.-H., Cosmovici, C.B., Mack, P., Craubner, H., and Schwarz, G. (1987) 'Dust coma morphology of comet Halley during the Giotto encounter', in E.J. Rolfe and B. Battrick (eds.), Diversity and Similarity of Comets, ESA SP-278, ESTEC, Noordwijk, pp. 271-275.

Ip, W.-H., and Rickman, H. (1986) 'A comparison of nucleus surface models to space observations of comet Halley', in O. Melita (ed.), Comet Nucleus Sample Return, ESA SP-249, ESTEC, Noordwijk, pp. 181-184.

Jeffers, H.M. (1946) 'Comet notes', Publ. Astron. Soc. Pacific 58, 60-61.

Jewitt, D.C. (1990) 'The persistent coma of comet P/Schwassmann-Wachmann 1', Astrophys. $J .$, in press.

Jewitt, D.C., and Luu, J. (1989) 'A CCD portrait of comet P/Tempel 2', Astron. J. 97, 1766-1790, 1840-1841.

Jewitt, D.C., and Meech, K.J. (1986) 'Cometary grain scattering versus wavelength, or, "What color is comet dust?" ', Astrophys. J. 310, 937-952.

Jewitt, D.C., and Meech, K.J. (1987) 'CCD photometry of comet P/Encke', Astron. J. 93, 1542-1548.

Jewitt, D.C., and Meech, K.J. (1988a) 'The absence of a color-distance trend in comets', Astron. J. 96, 1723-1730.

Jewitt, D.C., and Meech, K.J. (1988b) 'Optical properties of cometary nuclei and a preliminary comparison with asteroids', Astrophys. J. 328, 974-986.

Johnson, R.E., Cooper, J.F., Lanzerotti, L.J., and Strazzulla, G. (1987) 'Radiation formation of a non-volatile comet crust', Astron. Astrophys. 187, 889-892.

Jones, A.F. (1985) 'Periodic comet Encke (1961 I $=1974 \mathrm{~V}=1977 \mathrm{XI})$ ', Int. Comet $Q .7$, 98.

Kamél, L. (1989) 'The secular evolution of P/Encke's light curve: No fading, no perihelion asymmetry?', Icarus, submitted.

Kamoun, P.D., Campbell, D.B., Ostro, S.J., Pettengill, G.H., and Shapiro, I.I. (1982) 'Comet Encke: Radar detection of nucleus', Science 216, 293-296.

Kaneda, E., Ashihara, O., Shimizu, M., Takagi, M., and Hirao, K. (1986) 'Observation of comet Halley by the ultraviolet imager of Suisei', Nature 321, 297-299.

Keller, H.U., Arpigny, C., Barbieri, C., Bonnet, R.M., Cazes, S., Coradini, M., Cosmovici, C.B., Delamere, W.A., Huebner, W.F., Hughes, D.W., Jamar, C., Malaise, D., Reitsema, H.J., Schmidt, H.U., Schmidt, W.K.H., Seige, P., Whipple, F.L., and Wilhelm, K. (1986) 'First Halley Multicolour Camera imaging results from Giotto', Nature 321, 320-326.

Keller, H.U., Delamere, W.A., Huebner, W.F., Reitsema, H.J., Schmidt, H.U., Whipple, F.L., Wilhelm, K., Curdt, W., Kramm, R., Thomas, N., Arpigny, C., Barbieri, C., Bonnet, R.M., Cazes, S., Coradini, M., Cosmovici, C.B., Hughes, D.W., Jamar, C., Malaise, D., Schmidt, K., Schmidt, W.K.H., and Seige, P. (1987) 'Comet P/Halley's nucleus and its activity', Astron. Astrophys. 187, 807-823.

Kitamura, Y. (1986) 'Axisymmetric dusty gas jet in the inner coma of a comet', Icarus 66, 241-257.

Kitamura, Y. (1987) 'Axisymmetric dusty gas jet in the inner coma of a comet. II. The case of isolated jets', Icarus 72, 555-567. 
Klinger, J. (1983) 'Modelling of cometary nuclei', in C.-I. Lagerkvist and H. Rickman (eds.), Asteroids, Comets, Meteors, University of Uppsala, Uppsala, pp. 205-213.

Klinger, J. (1986) 'Possible alterations of matter in periodic comets', in O. Melita (ed.), Comet Nucleus Sample Return, ESA SP-249, ESTEC, Noordwijk, pp. 69-74.

Kochan, H., Feuerbacher, B., Joo, F., Klinger, J., Seboldt, W., Bischoff, A., Düren, H., Stöffler, D., Spohn, T., Fechtig, H., Grün, E., Kohl, H., Krankowsky, D., Roessler, K., Thiel, K., Schwehm, G., and Weisshaupt, U. (1989) 'Comet simulation experiments in the DFVLR space simulators', Adv. Space Res. 9, No. 3, pp. 113-122.

Kömle, N.I., and Ip, W.-H. (1987a) 'Anisotropic non-stationary gas flow dynamics in the coma of comet P/Halley', Astron. Astrophys. 187, 405-410.

Kömle, N.I., and Ip, W.-H. (1987b) 'A model of the anisotropic structure of the neutral gas coma of a comet', in E.J. Rolfe and B. Battrick (eds.), Diversity and Similarity of Comets, ESA SP-278, ESTEC, Noordwijk, pp. 247-254.

Krasnopolsky, V.A., Moroz, V.I., Krysko, A.A., Tkachuk, A.Y., Moreels, G., Clairemidi, J., Parisot, J.P., Gogoshev, M., and Gogosheva, T. (1987) 'Properties of dust in comet P/Halley measured by the VEGA-2 three-channel spectrometer', Astron. Astrophys. 187, 707-711.

Kresák, L. (1987) 'Dormant phases in the aging of periodic comets', Astron. Astrophys. 187, 906-908.

Kührt, E. (1984) 'Temperature profiles and thermal stresses in cometary nuclei', Icarus 60, 512-521.

Lamy, P. (1986) 'Ground-based observations of the dust emission from comet Halley', Adv. Space Res. 5, No. 12, pp. 317-323.

Landgraf, W. (1986) 'On the motion of comet Halley', Astron. Astrophys. 163, 246-260.

Larson, H.P., Davis, D.S., Mumma, M.J., and Weaver, H.A. (1986) 'Velocity-resolved observations of water in comet Halley', Astrophys. J. 309, L95-L99.

Larson, H.P., Mumma, M.J., and Weaver, H.A. (1987) 'Kinematic properties of the neutral gas outflow from comet P/Halley', Astron. Astrophys. 187, 391-397.

Larson, S.M. (1978) 'A rotation model for the spiral structure in the coma of comet Bennett (1970 II)', Bull. Am. Astron. Soc. 10, 589. (Abstract.)

Larson, S.M. (1980) 'CO' ${ }^{+}$in comet Schwassmann-Wachmann 1 near minimum brightness', Astrophys. J. 238, L47-L48.

Larson, S.M. (1986) 'A review of some digital image processing in cometary research', in C.-I. Lagerkvist, B.A. Lindblad, H. Lundstedt, and H. Rickman (eds.), Asteroids, Comets, Meteors II, University of Uppsala, Uppsala, pp. 449-459.

Larson, S.M., and Minton, R.B. (1972) 'Photographic observations of comet Bennett, 1970 II', in G.P. Kuiper and E. Roemer (eds.), Comets: Scientific Data and Missions, University of Arizona, Tucson, pp. 183-208.

Larson, S.M., and Sekanina, Z. (1984a) 'Coma morphology and dust-emission pattern of periodic comet Halley. I. High-resolution images taken at Mount Wilson in 1910', Astron. J. 89, 571-578, 600-606.

Larson, S.M., and Sekanina, Z. (1984b) 'Near-nucleus studies of comet Halley', in D.K. Yeomans, R.M. West, R.S. Harrington, and B.G. Marsden (eds.), Cometary Astrometry, JPL Publ. 84-82, Jet Propulsion Laboratory, Pasadena, pp. 14-20.

Larson, S.M., and Sekanina, Z. (1985) 'Coma morphology and dust-emission pattern of periodic comet Halley. III. Additional high-resolution images taken in 1910', Astron. J. 90, 823-826, 917-923. 
Larson, S.M., Sekanina, Z., Levy, D., Tapia, S., and Senay, M. (1987) 'Comet P/Halley near-nucleus phenomena in 1986', Astron. Astrophys. 187, 639-644.

Luu, J.X., and Jewitt, D.C. (1988) 'CCD investigation of comet P/Tempel 1', Bull. Am. Astron. Soc. 20, 835. (Abstract.)

Lynch, D.K., Russell, R.W., Retig, D.A., Rice, C.J., and Young, R.M. (1986) 'Pre-perihelion observations of comet Halley at 2-13 $\mu \mathrm{m}$ ', in B. Battrick, E.J. Rolfe, and R. Reinhard (eds.), Exploration of Halley's Comet, ESA SP-250, ESTEC, Noordwijk, vol. 3, pp. 479484.

Maas, D., Krueger, F.R., and Kissel, J. (1989) 'Mass and density of silicate- and CHONtype dust particles released by comet P/Halley', in C.-I. Lagerkvist, B.A. Lindblad, and H. Rickman (eds.), Asteroids, Comets, Meteors III, in press.

Marconi, M.L., and Mendis, D.A. (1986) 'Infrared heating of comet Halley's atmosphere', Earth Moon Planets 36, 249-256.

Marsden, B.G. (1969) 'Comets and nongravitational forces. II', Astron. J. 74, 720-734.

Marsden, B.G. (1970) 'Comets and nongravitational forces. III', A stron. J. 75, 75-84.

Marsden, B.G. (1972) 'Nongravitational effects on comets: The current status', in G.A. Chebotarev, E.I. Kazimirchak-Polonskaya, and B.G. Marsden (eds.), The Motion, Evolution of Orbits, and Origin of Comets, Reidel, Dordrecht, pp. 135-143.

Marsden, B.G. (1986) Catalogue of Cometary Orbits (5th edition), International Astronomical Union, Cambridge, MA, 102 pp.

Marsden, B.G., and Sekanina, Z. (1974) 'Comets and nongravitational forces. VI. Periodic comet Encke 1786-1971', Astron. J. 79, 413-419.

Marsden, B.G., Sekanina, Z., and Yeomans, D.K. (1973) 'Comets and nongravitational forces. V', Astron. J. 78, 211-225.

Massonne, L. (1986) 'Coma morphology and dust emission pattern of comet Halley', Adv. Space Res. 5, No. 12, pp. 187-196.

Massonne, L., Fertig, J., Grün, E., and Schwehm, G. (1986) 'A cometary dust environment model for the generation of synthetic images', in C.-I. Lagerkvist, B.A. Lindblad, H. Lundstedt, and H. Rickman (eds.), Asteroids, Comets, Meteors II, University of Uppsala, Uppsala, pp. 407-410.

Massonne, L., and Grün, E. (1986) 'Structures in Halley's dust coma', in B. Battrick, E.J. Rolfe, and R. Reinhard (eds.), Exploration of Halley's Comet, ESA SP-250, ESTEC, Noordwijk, vol. 3, pp. 319-322.

Mayall, N.U. (1941) 'Note on the spectrum of comet Schwassmann-Wachmann I (1925b)', Publ. Astron. Soc. Pacific 53, 340-341.

Mazets, E.P., Sagdeev, R.Z., Aptekar, R.L., Golenetskii, S.V., Guryan, Yu.A., Dyachkov, A.V., Ilyinskii, V.N., Panov, V.N., Petrov, G.G., Savvin, A.V., Sokolov, I.A., Frederiks, D.D., Khavenson, N.G., Shapiro, V.D., and Shevchenko, V.I. (1987) 'Dust in comet P/Halley from VEGA observations', Astron. Astrophys. 187, 699-706.

McDonnell, J.A.M., Alexander, W.M., Burton, W.M., Bussoletti, E., Evans, G.C., Evans, S.T., Firth, J.G., Grard, R.J.L., Green, S.F., Grün, E., Hanner, M.S., Hughes, D.W., Igenbergs, E., Kissel, J., Kuczera, H., Lindblad, B.A., Langevin, Y., Mandeville, J.-C., Nappo, S., Pankiewicz, G.S.A., Perry, C. H., Schwehm, G.H., Sekanina, Z., Stevenson, T.J., Turner, R.F., Weishaupt, U., Wallis, M.K., and Zarnecki, J.C. (1987) 'The dust distribution within the inner coma of comet P/Halley 1982i: Encounter by Giotto's impact detectors', Astron. Astrophys. 187, 719-741.

McFadden, L.A., A'Hearn, M.F., Feldman, P.D., Roettger, E.E., Edsall, D.M., and Butter- 
worth, P.S. (1987) 'Activity of P/Halley 23-25 March, 1986: IUE observations', Astron. Astrophys. 187, 333-338.

Meech, K.J., and Belton, M.J.S. (1989) '(2060) Chiron', IAU Circ. No. 4770.

Millis, R.L., A'Hearn, M.F., and Campins, H. (1988) 'An investigation of the nucleus and coma of comet P/Arend-Rigaux', Astrophys. J. 324, 1194-1209.

Millis, R.L., and Schleicher, D.G. (1986) 'Rotational period of comet Halley', Nature 324, 646-649.

Nakano, S. (1987) 'Periodic comet Encke', Minor Planet Circ. No. 12577.

Newburn, R.L., Jr., and Spinrad, H. (1989) 'Spectrophotometry of 25 comets: Post-Halley updates for 17 comets plus new observations for eight additional comets', Astron. J. 97, 552-569.

Öpik, E.J. (1958) Physics of Meteor Flight in the Atmosphere, Interscience, New York, $174 \mathrm{pp}$.

Palmer, P., de Pater, I., and Snyder, L.E. (1989) 'VLA observations of the OH emission from comet Wilson (1986l): The value of high resolution in both spatial and velocity coordinates', Astron. J. 97, 1791-1797, 1842.

Pankiewicz, G.S., and McDonnell, J.A.M. (1986) 'Dust emission studies in Halley's inner coma: An approach to modelling the nucleus emission characteristics', in B. Battrick, E.J. Rolfe, and R. Reinhard (eds.), Exploration of Halley's Comet, ESA SP-250, ESTEC, Noordwijk, vol. 2, pp. 201-206.

Patashnick, H., Rupprecht, G., and Schuerman, D.W. (1974) 'Energy source for comet outbursts', Nature 250, 313-314.

Pittich, E.M. (1972) 'Splitting and sudden outbursts of comets as indicators of nongravitational effects', in G.A. Chebotarev, E.I. Kazimirchak-Polonskaya, and B.G. Marsden (eds.), The Motion, Evolution of Orbits, and Origin of Comets, Reidel, Dordrecht, pp. 283-286.

Prialnik, D., and Bar-Nun, A. (1987) 'On the evolution and activity of cometary nuclei', Astrophys. J. 313, 893-905.

Rabinowitz, D.L. (1988) 'A source map for dust jets observed in the coma of comet P/Halley', Astron. Astrophys. 200, 225-247.

Rahe, J., Donn, B., and Wurm, K. (1969) Atlas of Cometary Forms, NASA SP-198, U.S. GPO, Washington, D.C., 128 pp.

Reitsema, H.J., Delamere, W.A., Williams, A.R., Boice, D.C., Huebner, W.F., and Whipple, F.L. (1989) 'Dust distribution in the inner coma of comet Halley: Comparison with models', Icarus 81, 31-40.

Rettig, T.W., Kern, J.R., Ruchti, R., Baumbaugh, B., Baumbaugh, A.E., Knickerbocker, K.L., and Dawe, J. (1987) 'Analysis of the coma outburst of comet Halley March 24-25 (UT), 1986', in E.J. Rolfe and B. Battrick (eds.), Diversity and Similarity of Comets, ESA SP-278, ESTEC, Noordwijk, pp. 265-269.

Riccò, A. (1912) 'Osservazioni astrofisiche della cometa Halley', Mem. Soc. Spettrosc. Ital. (Ser. 2) 1, 97-111.

Richter, N. (1941) 'Helligkeitsschwankungen der Kometen und Sonnentätigkeit. I. Erster Schwassmann-Wachmannscher Komet (1925 II)', Astron. Nachr. 271, 207-215.

Richter, N. (1948) 'Objekt Keuskamp und das Problem der unbestätigten Kometenentdeckungen', Sterne 24, 52-54.

Richter, N. (1954) 'Die Helligkeitsausbrüche des Kometen 1925 II und ihre Zusammenhänge mit der Sonnentätigkeit', Astron. Nachr. 281, 241-254. 
Rickman, H. (1986) 'Masses and densities of comets Halley and Kopff', in O. Melita (ed.), Comet Nucleus Sample Return, ESA SP-249, ESTEC, Noordwijk, pp. 195-205.

Rickman, H. (1989) 'The nucleus of comet Halley: Surface structure, mean density, gas and dust production', Adv. Space Res. 9, No. 3, pp. 59-71.

Rickman, H., and Fernández, J.A. (1986) 'Formation and blowoff of a cometary dust mantle', in O. Melita (ed.), Comet Nucleus Sample Return, ESA SP-249, ESTEC, Noordwijk, pp. 185-194.

Rickman, H., and Froeschlé, C. (1983) 'Model calculations of nongravitational effects on comet P/Halley', in T.I. Gombosi (ed.), Cometary Exploration, Hungarian Academy of Sciences, Budapest, vol. 3, pp. 109-126.

Rickman, H., and Froeschlé, C. (1986) 'Model calculations of nongravitational forces on short-period comets. II. High-obliquity case', Astron. Astrophys. 170, 161-166.

Rickman, H., Kamél, L., Festou, M.C., and Froeschlé, C. (1987) 'Estimates of masses, volumes and densities of short-period comet nuclei', in E.J. Rolfe and B. Battrick (eds.), Diversity and Similarity of Comets, ESA SP-278, ESTEC, Noordwijk, pp. 471-481.

Roemer, E. (1958) 'An outburst of comet Schwassmann-Wachmann 1', Publ. Astron. Soc. Pacific 70, 272-278.

Roemer, E. (1962) 'Activity in comets at large heliocentric distance', Publ. Astron. Soc. Pacific 74, 351-365.

Roemer, E., Thomas, M., and Lloyd, R.E. (1966) 'Observations of comets, minor planets, and Jupiter VIII', Astron. J. 71, 591-601.

Sagdeev, R.Z., Elyasberg, P.E., and Moroz, V.I. (1989) 'Is the nucleus of comet Halley a low density body?', Nature 331, 240-242.

Schloerb, F.P., Claussen, M.J., and Tacconi-Garman, L. (1987) 'OH radio observations of comet P/Halley', Astron. Astrophys. 187, 469-474.

Schlosser, W., Schulz, R., and Koczet, P. (1986) 'The cyan shells of comet P/Halley', in B. Battrick, E.J. Rolfe, and R. Reinhard (eds.), Exploration of Halley's Comet, ESA SP-250, ESTEC, Noordwijk, vol. 3, pp. 495-498.

Schulz, R., and Schlosser, W. (1989) ' $\mathrm{CN}$-shell structures and dynamics of the nucleus of comet P/Halley', Astron. Astrophys. 214, 375-385.

Sekanina, Z. (1969) 'Dynamical and evolutionary aspects of gradual deactivation and disintegration of short-period comets', Astron. J. 74, 1223-1234.

Sekanina, Z. (1972a) 'A model for the nucleus of Encke's comet', in G.A. Chebotarev, E.I. Kazimirchak-Polonskaya, and B.G. Marsden (eds.), The Motion, Evolution of Orbits, and Origin of Comets, Reidel, Dordrecht, pp. 301-307.

Sekanina, Z. (1972b) 'Rotation effects in the nongravitational parameters of comets', in G.A. Chebotarev, E.I. Kazimirchak-Polonskaya, and B.G. Marsden (eds.), The Motion, Evolution of Orbits, and Origin of Comets, Reidel, Dordrecht, pp. 294-300.

Sekanina, Z. (1979) 'Fan-shaped coma, orientation of rotation axis, and surface structure of a cometary nucleus', Icarus 37, 420-442.

Sekanina, Z. (1980) 'Physical characteristics of cometary dust from dynamical studies: A review', in I. Halliday and B.A. McIntosh (eds.), Solid Particles in the Solar System, Reidel, Dordrecht, pp. 237-250.

Sekanina, Z. (1981a) 'Rotation and precession of cometary nuclei', Ann. Rev. Earth Planet. Sci. 9, 113-145.

Sekanina, Z. (1981b) 'Distribution and activity of discrete emission areas on the nucleus of periodic comet Swift-Tuttle', Astron. J. 86, 1741-1773. 
Sekanina, Z. (1982) 'The problem of split comets in review', in L.L. Wilkening (ed.), Comets, University of Arizona, Tucson, pp. 251-287.

Sekanina, Z. (1984a) 'Disappearance and disintegration of comets', Icarus 58, 81-100.

Sekanina, Z. (1984b) 'Precession model for the nucleus of periodic comet Kopff', Astron. J. $89,1573-1586$.

Sekanina, Z. (1985a) 'Precession model for the nucleus of periodic comet Giacobini-Zinner', Astron. J. 90, 827-845.

Sekanina, Z. (1985b) 'Nucleus precession of periodic comet Comas-Solá', Astron. J. 90, 1370-1381. Adv. Space Res. 5, No. 12, pp. 307-316.

Sekanina, Z. (1986a) 'Nucleus studies of comet Halley', Adv. Space Res. 5, No. 12, pp. 307-316.

Sekanina, Z. (1986b) 'Comet Halley (1982i)', IAU Circ. No. 4187.

Sekanina, Z. (1987a) 'Dust environment of comet P/Halley: A review', Astron. Astrophys. 187, 789-795.

Sekanina, Z. (1987b) 'Anisotropic emission from comets: Fans versus jets. I. Concept and modeling', in E.J. Rolfe and B. Battrick (eds.), Diversity and Similarity of Comets, ESA SP-278, ESTEC, Noordwijk, pp. 315-322.

Sekanina, Z. (1987c) 'Anisotropic emission from comets: Fans versus jets. II. Periodic comet Tempel 2', in E.J. Rolfe and B. Battrick (eds.), Diversity and Similarity of Comets, ESA SP-278, ESTEC, Noordwijk, pp. 323-336.

Sekanina, Z. (1988a) 'Nucleus of comet IRAS-Araki-Alcock (1983 VII)', Astron. J. 95, 1876-1894.

Sekanina, Z. (1988b) 'Outgassing asymmetry of periodic comet Encke. I. Apparitions 19241984', Astron. J. 95, 911-924, 970-971.

Sekanina, Z. (1988c) 'Outgassing asymmetry of periodic comet Encke. II. Apparitions 18681918 and a study of the nucleus evolution', Astron. J. 96, 1455-1475, 1508.

Sekanina, Z. (1988d) 'Periodic comet Tempel 2 (1987g)', IAU Circ. No. 4624.

Sekanina, Z. (1989) 'Nuclei of two Earth-grazing comets of fan-shaped appearance', Astron. J. 98, 2322-2345.

Sekanina, Z. (1990) 'Periodic comet Machholz and its idiosyncrasies', Astron. J., in press.

Sekanina, Z., and Farrell, J.A. (1978) 'Comet West 1976 VI: Discrete bursts of dust, split nucleus, flare-ups, and particle evaporation', Astron. J. 83, 1675-1680.

Sekanina, Z., and Larson, S.M. (1983) 'Rotating comets: Development of dust spirals into envelopes', Bull. Am. Astron. Soc. 15, 651. (Abstract.)

Sekanina, Z., and Larson, S.M. (1984) 'Coma morphology and dust-emission pattern of periodic comet Halley. II. Nucleus spin vector and modeling of major dust features in 1910', Astron. J. 89, 1408-1425, 1446-1447.

Sekanina, Z., and Larson, S.M. (1986a) 'Coma morphology and dust-emission pattern of periodic comet Halley. IV. Spin vector refinement and map of discrete dust sources for 1910', Astron. J. 92, 462-482.

Sekanina, Z., and Larson, S.M. (1986b) 'Dust jets in comet Halley observed by Giotto and from the ground', Nature 321, 357-361.

Sekanina, Z., and Yeomans, D.K. (1985) 'Orbital motion, nucleus precession, and splitting of periodic comet Brooks 2', Astron. J. 90, 2335-2352.

Shao, C.-Y. (1981) 'On the outbursts of P/comet Schwassmann-Wachmann 1 during 19801981', Int. Comet Q. 3, 76.

Shao, C.-Y., and Schwartz, G. (1981) 'Thar she blows-again!', Sky Telesc. 61, 390. 
Shimizu, M. (1976) 'The structure of cometary atmospheres. I. Temperature distribution', Astrophys. Space Sci. 40, 149-155.

Shulman, L.M. (1972) 'The evolution of cometary nuclei', in G.A. Chebotarev, E.I. Kazimirchak-Polonskaya, and B.G. Marsden, The Motion, Evolution of Orbits, and Origin of Comets, Reidel, Dordrecht, pp. 271-282.

Silva, A.M., and Mirabel, I.F. (1988) 'Gaseous outbursts in comet P/Halley. A model for the dissociation of the OH radical', Astron. Astrophys. 201, 350-354.

Simpson, J.A., Rabinowitz, D., Tuzzolino, A.J., Ksanfomality, L.V., and Sagdeev, R.Z. (1987) 'The dust coma of comet P/Halley: Measurements on the VEGA-1 and VEGA-2 spacecraft', Astron. Astrophys. 187, 742-752.

Sitarski, G. (1987) 'Linkage of the 53 observed perihelion times of the periodic comet Encke', Acta Astron. 37, 99-113.

Smirnov, V.N., Vaisberg, O.L., and Anisimov, S. (1987) 'An attempt to evaluate the structure of cometary dust particles', Astron. Astrophys. 187, 774-778.

Smoluchowski, R. (1981a) 'Amorphous ice and the behavior of cometary nuclei', Astrophys. J. 244, L31-L34.

Smoluchowski, R. (1981b) 'Heat content and evolution of cometary nuclei', Icarus 47, 312319.

Smoluchowski, R. (1982) 'Heat transport in porous cometary nuclei', J. Geophys. Res. 87, A422-A424.

Smoluchowski, R. (1986) 'Brightness curve and porosity of cometary nuclei', in C.-I. Lagerkvist, B.A. Lindblad, H. Lundstedt, and H. Rickman (eds.), Asteroids, Comets, Meteors II, University of Uppsala, Uppsala, pp. 305-315.

Smoluchowski, R. (1989) 'Properties of mantles on cometary nuclei', Astron. J. 97, 241-245.

Snyder, L.E., Webber, J.C., Crutcher, R.M., and Swenson, G.W., Jr. (1976) 'Radio observations of $\mathrm{OH}$ in comet West 1975n', Astrophys. J. 209, L49-L52.

Spohn, T., Benkhoff, J., Klinger, J., Grün, E., and Kochan, H. (1989) 'Thermal modelling of two KOSI comet nucleus simulation experiments', Adv. Space Res. 9, No. 3, pp. 127-131.

Tauber, F., and Kührt, E. (1987) 'Thermal stresses in cometary nuclei', Icarus 69, 83-90.

Thomas, N., Boice, D.C., Huebner, W.F., and Keller, H.U. (1988) 'Intensity profiles of dust near extended sources on comet Halley', Nature 332, 51-52.

Thomas, N., and Keller, H.U. (1987) 'Fine dust structures in the emission of comet P/Halley observed by the Halley Multicolour Camera on board Giotto', Astron. Astrophys. 187, 843-846.

Vaisberg, O.L., Smirnov, V., Omelchenko, A., Gorn, L., and Iovlev, M. (1987) 'Spatial and mass distribution of low-mass dust particles $\left(m<10^{-10} \mathrm{~g}\right)$ in comet P/Halley's coma', Astron. Astrophys. 187, 753-760.

Van Biesbroeck, G. (1921) 'Observations of comets', A stron. J. 33, 195-200.

Vergazov, M.A., and Krasnobaev, K.V. (1985) 'Axisymmetric flow of reacting gas from a comet nucleus', Sov. Astron. Lett. 11, 232-235.

Walker, M.F. (1959) 'Observations of comet Schwassmann-Wachmann 1 during an outburst', Publ. Astron. Soc. Pacific 71, 169-170.

Wallis, M.K., and Macpherson, A.K. (1981) 'On the outgassing and jet thrust of snowball comets', Astron. Astrophys. 98, 45-49.

Wallis, M.K., and Wickramasinghe, N.C. (1987) 'Crustal models of the evolving comet nucleus', in E.J. Rolfe and B. Battrick (eds.), Diversity and Similarity of Comets, ESA SP-278, ESTEC, Noordwijk, pp. 495-499. 
Weaver, H.A., Feldman, P.D., Festou, M.C., A'Hearn, M.F., and Keller, H.U. (1981) 'IUE observations of faint comets', Icarus 47, 449-463.

Weaver, H.A., Mumma, M.J., and Larson, H.P. (1987) 'Infrared investigation of water in comet P/Halley', Astron. Astrophys. 187, 411-418.

Weaver, H.A., Mumma, M.J., Larson, H.P., and Davis, D.S. (1986) 'Post-perihelion observations of water in comet Halley', Nature 324, 441-444.

Weigert, A. (1959) 'Halobildung beim Kometen 1925 II', Astron. Nachr. 285, 117-128.

Weissman, P.R. (1986) 'Are cometary nuclei primordial rubble piles?', Nature 320, 242-244.

Weissman, P.R. (1987) 'Post-perihelion brightening of comet P/Halley: Springtime for Halley', Astron. Astrophys. 187, 873-878.

Whipple, F.L. (1950) 'A comet model. I. The acceleration of comet Encke', Astrophys. J. 111, 375-394.

Whipple, F.L. (1951) 'A comet model. II. Physical relations for comets and meteors', Astrophys. J. 113, 464-474.

Whipple, F.L. (1977) 'The constitution of cometary nuclei', in A.H. Delsemme (ed.), Comets, A steroids, Meteorites, University of Toledo, Toledo, Ohio, pp. 25-35.

Whipple, F.L. (1978) 'Rotation period of comet Donati', Nature 273, 134-135.

Whipple, F.L. (1980) 'Rotation and outbursts of comet Schwassmann-Wachmann 1', Astron. J. 85, 305-313.

Whipple, F.L. (1984) 'Comet P/Holmes, 1892 III: A case of duplicity?', Icarus 60, 522-531.

Whipple, F.L., and Hamid, S.E. (1952) 'On the origin of the Taurid meteor streams', Helwan Obs. Bull. No. 41, pp. 1-30.

Whipple, F.L., and Hamid, S.E. (1972) 'A search for Encke's comet in ancient Chinese records: A progress report', in G.A. Chebotarev, E.I. Kazimirchak-Polonskaya, and B.G. Marsden (eds.), The Motion, Evolution of Orbits, and Origin of Comets, Reidel, Dordrecht, pp. 152-154.

Whipple, F.L., and Sekanina, Z. (1979) 'Comet Encke: Precession of the spin axis, nongravitational motion, and sublimation', Astron. J. 84, 1894-1909.

Whitney, C. (1955) 'Comet outbursts', A strophys. J. 122, 190-195.

Wilhelm, K., Cosmovici, C.B., Delamere, W.A., Huebner, W.F., Keller, H.U., Reitsema, H., Schmidt, H.U., and Whipple, F.L. (1986) 'A three-dimensional model of the nucleus of comet Halley', in B. Battrick, E.J. Rolfe, and R. Reinhard (eds.), Exploration of Halley's Comet, ESA SP-250, ESTEC, Noordwijk, vol. 2, pp. 367-369.

Winnecke, A. (1864) 'Pulkowaer Beobachtungen des hellen Cometen von 1862', Mém. Acad. Imp. Sci. St.-Pétersbourg 7 (Ser. 7), No. 7, pp. 1-39.

Yeomans, D.K. (1971) 'Nongravitational forces affecting the motion of periodic comets Giacobini-Zinner and Borrelly', Astron. J. 76, 83-86.

Yeomans, D.K. (1984) 'The orbits of comets Halley and Giacobini-Zinner', in D.K. Yeomans, R.M. West, R.S. Harrington, and B.G. Marsden (eds.), Cometary Astrometry, JPL Publ. 84-82, Jet Propulsion Laboratory, Pasadena, pp. 167-175.

Yeomans, D.K., and Chodas, P.W. (1989) 'An asymmetric outgassing model for cometary nongravitational accelerations', Astron. J. 98, 1083-1093.

Yeomans, D.K., and Kiang, T. (1981) 'The long-term motion of comet Halley', Mon. Not. R. Astron. Soc. 197, 633-646. 